\title{
STATIONARY MEASURES AND INVARIANT SUBSETS OF HOMOGENEOUS SPACES (II)
}

\author{
YVES BENOIST AND JEAN-FRANÇOIS QUINT
}

\section{Contents}

1. Introduction 659

2. Main results 663

3. Conditional measures 664

4. Random walks on semisimple groups $\quad 670$

5. Dynamics on homogeneous spaces 684

6. The first return Markov chain 701

7. Before the drift 718

8. Invariance of stationary probability measures $\quad 726$

References

\section{INTRODUCTION}

This text is part of a series of papers which aim at understanding some phenomena on the dynamics of subgroup actions on finite volume homogeneous spaces. In this introduction we describe our main result for real Lie groups. More general statements are given in Chapter 2

\subsection{Actions on $G / \Lambda$.}

Let $G$ be a real Lie group, $\Lambda$ be a lattice in $G, X=G / \Lambda$ and $\mu$ be a probability measure on $G$. We want to describe the $\mu$-stationary probability measures $\nu$ on $X$.

We recall that a probability measure $\nu$ on $X$ is said to be $\mu$-stationary if one has $\mu * \nu=\nu$. It is then said to be $\mu$-ergodic if it is extremal among $\mu$-stationary probability measures.

We will say that a probability measure $\nu$ on $X$ is homogeneous if it is supported by a closed orbit $F$ of its stabilizer $G_{\nu}:=\left\{g \in G \mid g_{*} \nu=\nu\right\}$. Such a probability is a finite average of probability measures which are homogeneous under the connected component of $G_{\nu}$.

Received by the editors July 8, 2011 and, in revised form, October 9, 2012.

2010 Mathematics Subject Classification. Primary 22E40, 37C40, 37C85.

(C) 2013 American Mathematical Society Reverts to public domain 28 years from publication 
Let $\Gamma_{\mu}$ be the subgroup of $G$ generated by the support of $\mu$. Let $\mathfrak{g}$ be the Lie algebra of $G$ and $H_{\mu}:={\overline{\operatorname{Ad}\left(\Gamma_{\mu}\right)}}^{Z} \subset \mathrm{GL}(\mathfrak{g})$ be the Zariski closure of the adjoint $\operatorname{group} \operatorname{Ad}\left(\Gamma_{\mu}\right)$.

Theorem 1.1. Let $G$ be a real Lie group, $\Lambda$ be a lattice in $G, X=G / \Lambda$ and $\mu$ be a compactly supported probability measure on $G$ such that the group $H_{\mu}:={\overline{\operatorname{Ad}\left(\Gamma_{\mu}\right)}}^{Z}$ is semisimple and Zariski connected with no compact factor. Then every $\mu$-ergodic $\mu$-stationary probability measure $\nu$ on $X$ is $\Gamma_{\mu}$-invariant and homogeneous.

Note that it is easy to describe these $\Gamma_{\mu}$-invariant homogeneous probability measures $\nu$ on $G$ using the following remarks: since their stabilizer $G_{\nu}$ is a closed subgroup of $G$ containing $\Gamma_{\mu}$, the connected component $S$ of $G_{\nu}$ is a subgroup of $G$ whose Lie algebra is stable under the action of $H_{\mu}$, and, since $\nu$ is $\mu$-ergodic, one has $G_{\nu}=\Gamma_{\mu} S$.

When $H_{\mu}$ is not assumed to be Zariski connected and is allowed to have nontrivial compact factors, we can still describe all the $\mu$-ergodic $\mu$-stationary measures on $X$ even though they might be non-homogeneous. Such examples are called satellite probability measures (see [6]).

Corollary 1.2. Let $G$ be a connected semisimple real algebraic group with no compact factor, $\Lambda$ be an irreducible lattice in $G$ and $\mu$ be a probability measure on $G$ whose support is compact and spans a Zariski dense subgroup of $G$. Then every non-atomic $\mu$-stationary probability measure on $X$ is $G$-invariant.

Under the stronger assumption that $G$ is simple, Corollary 1.2 is the first main result of $[4$.

\subsection{Actions on tori and nilmanifolds.}

We now describe the applications of our Theorem 1.1 to the affine actions on tori and more generally to the affine actions on nilmanifolds.

Let $N$ be a connected simply connected nilpotent real Lie group, $\Lambda$ be a lattice in $N$ and $X$ be the compact nilmanifold $X=N / \Lambda$. By an affine submanifold of $X$, we shall mean a closed subset of $X$ which is an orbit under a connected subgroup of $N$. By Mal'cev's rigidity theorem (see [24, II.2.11]), the discrete group $\operatorname{Aut}(\Lambda)$ of automorphisms of $\Lambda$ embeds in a natural way in the group of automorphisms of $N$. Define the group of affine transformations of $X$ to be the semidirect product $G:=$ $\operatorname{Aff}(X)=\operatorname{Aut}(\Lambda) \ltimes N / Z_{\Lambda}$, where $Z_{\Lambda}$ is the center of $\Lambda$. Then $G$ acts transitively on $X$ and we may see $X$ as the quotient of $G$ by the lattice $\operatorname{Aut}(\Lambda) \ltimes \Lambda / Z_{\Lambda}$. The action of $\operatorname{Aut}(\Lambda) \subset G$ by left translations on this quotient is its usual action on $X$. In this case, a homogeneous probability measure on $X$ is a finite average of Haar probability measures carried by affine submanifolds of $X$.

For example, if $N=\mathbb{R}^{d}$ and $\Lambda=\mathbb{Z}^{d}$, one has $X=\mathbb{T}^{d}, G=\operatorname{Aff}(X)=\operatorname{GL}(d, \mathbb{Z}) \ltimes$ $\mathbb{T}^{d}$ and $\operatorname{Aut}(\Lambda)=\mathrm{GL}(d, \mathbb{Z}) \subset \operatorname{Aut}(N)=\mathrm{GL}(d, \mathbb{R})$.

By Theorem 1.1, we get the following corollary which gives a partial answer to [20, Problem 4]:

Corollary 1.3. Let $X=N / \Lambda$ be a compact nilmanifold, $\mu$ be a finitely supported probability measure on $\operatorname{Aut}(\Lambda)$ and $\Gamma_{\mu}$ be the subgroup generated by the support of $\mu$. We assume that the Zariski closure of $\Gamma_{\mu}$ in $\operatorname{Aut}(N)$ is semisimple and Zariski 
connected with no compact factor. Then every $\mu$-ergodic $\mu$-stationary probability measure $\nu$ on $X$ is $\Gamma_{\mu}$-invariant and homogeneous.

A description of some cases of Corollary 1.3 where $X$ is a Heisenberg nilmanifold has been obtained by J.-R. Heu in [19] based on [4].

Corollary 1.4. Let $X$ be the torus $X=\mathbb{T}^{d}$, $\mu$ be a finitely supported probability measure on $\mathrm{GL}(d, \mathbb{Z})$ and $\Gamma_{\mu}$ be the subgroup generated by the support of $\mu$. Assume the Zariski closure of $\Gamma_{\mu}$ is semisimple, Zariski connected and with no compact factor, and acts irreducibly on $\mathbb{Q}^{d}$. Then the only atom-free $\mu$-stationary probability measure on $\mathbb{T}^{d}$ is the Haar probability on $\mathbb{T}^{d}$.

To our knowledge, Corollary 1.4 was previously only known under the additional assumption that $\Gamma_{\mu}$ acts irreducibly on $\mathbb{R}^{d}$ : this is due to [12] in the proximal case and to 4$]$ in general. For instance, the action of the group $\operatorname{SL}(2, \mathbb{Z}[\sqrt{2}])$ is irreducible on $\mathbb{Q}^{4}$ but is not irreducible on $\mathbb{R}^{4}$.

\subsection{Strategy of the proof.}

Since the proof of Theorem 1.1 will last up to the end of this paper, we begin by a sketch of the main ideas.

As in 4, our approach is based on a study of the random walk induced by $\mu$ on $G / \Lambda$, that is, the Markov chain with transition probabilities $\mu * \delta_{x}, x \in G / \Lambda$. We still use the "exponential drift" argument based on the martingale convergence theorem that we introduced in [4] when $G$ is simple and $H_{\mu}=G$.

However, the strategy is different from the one followed in [4]: we do not use any suspension of Bernouilli shift. Instead, we modify the "exponential drift" argument by replacing the tail $\sigma$-algebra of the suspension by the tail $\sigma$-algebra of a fibered dynamical system whose fiber has infinite volume. More precisely, we first consider a finite volume fibered dynamical system $\left(B^{X}, \mathcal{B}^{X}, \beta^{X}, T^{X}\right)$ with fiber $X$ and with base the one-sided Bernoulli shift $(B, \mathcal{B}, \beta, T)$ with alphabet $(G, \mu)$.

Let $\mathfrak{g}=\mathfrak{l} \oplus \mathfrak{v}_{1} \oplus \cdots \oplus \mathfrak{v}_{\ell}$ be a decomposition of the Lie algebra of $G$, where $\mathfrak{l}$ is the centralizer of $\Gamma_{\mu}$ and where the $\mathfrak{v}_{i}$ 's are non-trivial irreducible $\Gamma_{\mu}$-modules. The case we were dealing with in $[4$ is the case where $\mathfrak{l}=0$ and $\ell=1$. Each of these representations $\mathfrak{v}_{i}$ gives us a function $\theta_{i}$ on $B$ describing the logarithmic growth of the random walk on $G$ in the most expanding direction of $\mathfrak{v}_{i}$.

We collect these functions as a function $\theta$ on $B$ with values in a locally compact group $Z$. This group $Z$ is a compact extension of a non-compact abelian group. We will consider the function $\theta$ as a cocycle on $\left(B^{X}, \mathcal{B}^{X}, \beta^{X}, T^{X}\right)$ and study the corresponding fibered dynamical system $\left(B^{\theta, X}, \mathcal{B}^{\theta, X}, \beta^{\theta, X}, T^{\theta, X}\right)$ with fiber $Z$. The study of this dynamical system needs some special care since the invariant measure $\beta^{\theta, X}$ has infinite volume. Hence we introduce an open subset $U$ in $Z$ of finite volume and the subspace $B^{U, X} \subset B^{\theta, X}$ with fiber $U$ over $B^{X}$.

We introduce the $\sigma$-subalgebras $\mathcal{Q}_{n}^{\theta, X}=\left(T^{\theta, X}\right)^{-n} \mathcal{B}^{\theta, X}$ whose intersection is the tail $\sigma$-algebra $\mathcal{Q}_{\infty}^{\theta, X}$. We also introduce the $\sigma$-algebras $\mathcal{Q}_{n}^{U, X}$ which are the restrictions of $\mathcal{Q}_{n}^{\theta, X}$ to $B^{U, X}$. These $\sigma$-algebras are very convenient to us for the following two reasons.

On one hand, in Chapter 3, we prove a very simple formula to express the conditional expectation $\varphi_{n}=\mathbb{E}\left(\varphi \mid \mathcal{Q}_{n}^{U, X}\right)$ of a measurable function $\varphi$ on $B^{U, X}$ with respect to this $\sigma$-algebra $\mathcal{Q}_{n}^{U, X}$. 
On the other hand, in Chapter 4, we are able to control the asymptotic behavior as $n$ goes to $\infty$ of the corresponding conditional probability measures $\beta_{n, c, x}^{U, X}$ thanks to the "law of the angles". To check this asymptotic law, we use a tricky combination of an iterated logarithm law, a local limit theorem and a large deviation estimate for random walks on semisimple groups that we have proved to this purpose in 9 .

Chapter 5 is a potpourri of preliminary results, including structure results for real and $p$-adic Lie groups and their lattices, which will be used later.

In Chapter 6 we prove a general phenomenon for the random walk on $X=G / \Lambda$ which we have called "positive $\mu$-unstability of the diagonal" and which is the main ingredient for Chapter 7 To check that this phenomenon holds when the space $X=G / \Lambda$ is not compact, we use the exponential $\mu$-recurrence of the random walk on $X=G / \Lambda$ that we have proven in 7 .

Chapter 7 contains two important ideas. First we check that if $\mu$ is non-atomic, $\beta$-almost surely, the limit probability measures $\nu_{b}$ are not supported by the "stable leaf" of an orbit of the centralizer $L$ of $\Gamma_{\mu}$. Second, we introduce the horocyclic flow and check that the "conditional measures of $\nu_{b}$ along the horocycle flow" map is measurable for the tail $\sigma$-algebra $\mathcal{Q}_{\infty}^{\theta, X}$. These two points will be crucial input in Chapter 8 .

In Chapter 8 we develop our new "exponential drift" argument. This argument should be familiar to a reader who already knows 4, even though the tools involved in the proof are more sophisticated than in [4. The main point is to accurately control the size and the direction of the drift. This control is the purpose of the "law of the angles" proven in Chapter 4. As in 4, the aim of the exponential drift argument is to show that almost surely the conditional measures of $\nu_{b}$ along the horocycle flow have a non-trivial stabilizer. This piece of information allows us to write $\nu$ as an average of non-trivial probability measures $\alpha$ which are invariant and ergodic under a one-parameter Ad-unipotent subgroup. By Ratner's theorem [25], such a probability measure is homogeneous. Thus, this construction provides us with a probability measure $\eta$ on the set $\mathcal{E}$ of probability measures which are homogeneous and ergodic under some one-parameter Ad-unipotent subgroup. By the equivariance properties of this construction, the probability measure $\eta$ is itself $\mu$-stationary and $\mu$-ergodic. By ergodicity this probability $\eta$ is supported by some $G$-orbit $G \alpha_{0}$ in $\mathcal{E}$. The key point is then to check, using one of the potpourri results of Chapter 5. that $\eta$ is supported by an orbit of the unimodular normalizer of the connected component $S_{\alpha_{0}}$ of the stabilizer of $\alpha_{0}$. We conclude then, by a kind of induction argument, that $\eta$ is homogeneous. Therefore $\nu$ is homogeneous, too.

The results stated in this introduction were announced in [5]. We will apply them in [6] to the classification of the $\Gamma_{\mu}$-orbit closures in $X$ which we also announced in [5].

Since our ergodic theorical method is very flexible, our results will be stronger than the statements in this introduction: we will work with products of real and $p$-adic Lie groups. This extension will lengthen this article by only 12 pages. We have gathered the extra $p$-adic techniques in sections 2.1, 2.2, 5.1, 5.2, 5.6, and 5.8, that a reader only interested in real Lie groups can avoid, and we have tried to keep notation for the other sections as light as if we were dealing only with real Lie groups. 


\section{MAIN RESUltS}

In this section we formulate our main result which generalizes Theorem 1.1. The reader who is only interested in real Lie groups may go directly to section 2.3 .

\subsection{Weakly regular $\mathcal{S}$-adic groups.}

Here we define a class of $\mathcal{S}$-adic Lie groups to which our results apply.

For any prime number $p$, let $\mathbb{Q}_{p}$ denote the field of $p$-adic numbers and $\mathbb{Q}_{\infty}=\mathbb{R}$ denote the field of real or " $\infty$-adic" numbers. Let $\{\infty, 2,3,5, \ldots\}$ be the set of prime numbers including $\infty$ and $\mathcal{S}$ be a finite subset of it. We let $\mathbb{Q}_{\mathcal{S}}$ be the locally compact algebra $\bigoplus_{p \in \mathcal{S}} \mathbb{Q}_{p}$.

Definition 2.1 (Ratner, [26]). A $p$-adic Lie group $G$ is said to be weakly regular if any two one-parameter subgroups $\mathbb{Q}_{p} \rightarrow G$ with the same derivative at $e$ are equal.

A weakly regular $\mathcal{S}$-adic Lie group $G$ is a locally compact group which is isomorphic to a closed subgroup of a product of weakly regular $p$-adic Lie groups, with $p \in \mathcal{S}$.

For instance, every real Lie group is weakly regular since the one-parameter subgroups are characterized by their derivative at $e$.

Example 2.2 ([26, Cor. 1.3 and Prop. 1.5]). Every closed subgroup of a finite product $\prod_{p \in \mathcal{S}} \mathrm{GL}\left(d_{p}, \mathbb{Q}_{p}\right)$ is a weakly regular $\mathcal{S}$-adic Lie group.

In this article, all the measures are assumed to be Borel measures.

Let $G$ be a weakly regular $\mathcal{S}$-adic Lie group, $\Lambda$ be a discrete subgroup of $G$ and $X=G / \Lambda$. Let $\mu$ be a probability measure on $G$. We denote by $\Gamma=\Gamma_{\mu}$ the closed subgroup generated by the support of $\mu$. Let $\mathfrak{g}=\bigoplus_{p \in \mathcal{S}} \mathfrak{g}_{p}$ be the Lie algebra of $G$ and Ad be the adjoint action of $G$ in $\mathfrak{g}$ (see section 5.1 for more details). We denote by $H_{\mu}:={\overline{\operatorname{Ad}\left(\Gamma_{\mu}\right)}}^{Z}$ the Zariski closure of the group $\operatorname{Ad}\left(\Gamma_{\mu}\right)$ in $\operatorname{GL}(\mathfrak{g})$. This means that $H_{\mu}$ is the smallest group containing $\mathrm{Ad} \Gamma_{\mu}$ which is a product $H_{\mu}:=\prod_{p \in \mathcal{S}} H_{\mu, p}$ of Zariski closed subgroups $H_{\mu, p} \subset \mathrm{GL}\left(\mathfrak{g}_{p}\right)$. Hence for every $p \in \mathcal{S}, H_{\mu, p}$ is the Zariski closure of the group $\operatorname{Ad}_{\mathfrak{g}_{p}} \Gamma_{\mu} \subset \mathrm{GL}\left(\mathfrak{g}_{p}\right)$. We will say that $H_{\mu}$ is semisimple if all the groups $H_{\mu, p}$ are semisimple, and we will always assume semisimple algebraic groups to be Zariski conneceted. We will say that

Definition 2.3. $\mu$ is Ad-semisimple if the group $H_{\mu}$ is semisimple.

Let $H_{\mu}^{n c} \subset H_{\mu}$ be the intersection of the kernels of the algebraic representations $\rho: H_{\mu} \rightarrow \mathrm{GL}\left(d, \mathbb{Q}_{\mathcal{S}}\right)$ for which $\rho\left(\operatorname{Ad}\left(\Gamma_{\mu}\right)\right)$ is bounded. When $\mu$ is Ad-semisimple and $\mathcal{S}=\{\infty\}$, one has the equivalence

$$
H_{\mu}=H_{\mu}^{n c} \Longleftrightarrow H_{\mu} \text { is Zariski connected with no compact factor. }
$$

In this paper, we will always assume that $\mu$ is Ad-semisimple. Often we will also assume that $H_{\mu}=H_{\mu}^{n c}$.

\subsection{Stationary probability measures.}

In this section we explain our classification of stationary probability measures.

Definition 2.4. A probability measure $\nu$ on $X$ is homogeneous if it is supported on a closed orbit of its stabilizer $G_{\nu}:=\left\{g \in G \mid g_{*} \nu=\nu\right\}$. 
Theorem 2.5. Let $G$ be a weakly regular $\mathcal{S}$-adic Lie group, $\Lambda$ be a lattice in $G$, $X=G / \Lambda$ and $\mu$ be an Ad-semisimple compactly supported probability measure on $G$ such that $H_{\mu}=H_{\mu}^{n c}$. Then every $\mu$-ergodic $\mu$-stationary probability measure $\nu$ on $X$ is $\Gamma_{\mu}$-invariant and homogeneous.

Corollary 2.6. Let $G$ be a finite product of Zariski connected linear semisimple $p$-adic Lie groups, $\Lambda$ be an irreducible lattice in $G, X=G / \Lambda$ and $\mu$ be a compactly supported probability measure on $G$. We assume that the image of $\Gamma_{\mu}$ in every non-trivial semisimple quotient of $G$ is Zariski dense and unbounded. Then every atom-free $\mu$-stationary probability measure on $X$ is invariant by some finite index subgroup $G^{\prime} \subset G$.

\subsection{The case where $\Lambda$ is not a lattice.}

Even when $G$ is a real Lie group, the reduction process of the proof of Theorem 2.5 will force us to also deal with discrete subgroups $\Lambda$ which are not lattices.

We choose a norm $\|\cdot\|$ on each of the $p$-adic Lie algebras $\mathfrak{g}_{p}$, and, for $v=\left(v_{p}\right)_{p \in \mathcal{S}}$ in $\mathfrak{g}$, we set $\|v\|:=\max _{p \in \mathcal{S}}\left\|v_{p}\right\|$. We pick a standard open subset $\Omega$ of $G$ with exponential map $\exp _{\Omega}: O \rightarrow \Omega$ (see section 5.1) where $O$ is a relatively compact open neighborhood of 0 in $\mathfrak{g}$. We fix $r_{0}>0$ such that $B\left(0, r_{0}\right) \subset O$, and, for $x \in X$, we define the injectivity radius at $x$ by

$$
r_{X}(x):=\max \left\{r \leq r_{0} \mid v \mapsto \exp _{\Omega}(v) x \text { is injective on } B(0, r)\right\} .
$$

A subset $F$ of $X$ is said to be exponentially $\mu$-recurrent if the sequence

$$
n \mapsto \sup _{x \in F}\left(\mu^{\otimes n}\left(\left\{\left(g_{1}, \ldots g_{n}\right) \in G^{n} \mid g_{j} \cdots g_{1} x \notin F \text { for all } j=1, \ldots, n\right\}\right)\right)
$$

decays exponentially (see Definition 6.1).

We say that the cusps of $X$ are exponentially $\mu$-unstable if every compact subset of $X$ is contained in an exponentially $\mu$-recurrent closed set $F$ on which the injectivity radius is bounded below (see Definition 6.20).

Proof of Theorem 2.5. This will follow from Theorem 2.7 since the main result of [7] tells us that, when $\Lambda$ is a lattice, the cusps of $X$ are exponentially $\mu$-unstable (see Lemma 6.21).

Theorem 2.7. Let $G$ be a weakly regular $\mathcal{S}$-adic Lie group, $\Lambda$ be a discrete subgroup of $G, X=G / \Lambda$, and $\mu$ be an Ad-semisimple compactly supported probability measure on $G$ such that $H_{\mu}=H_{\mu}^{n c}$. We assume that the cusps of $X$ are exponentially $\mu$-unstable. Then every $\mu$-ergodic $\mu$-stationary probability measure $\nu$ on $X$ is $\Gamma_{\mu}$-invariant and homogeneous.

Theorem 2.7 might be true for any discrete subgroup $\Lambda$ of $G$. Indeed, the cusps of $X$ might always be exponentially $\mu$-unstable. We checked this when $G$ is a semisimple real Lie group and $\Gamma_{\mu}$ is a Zariski dense subgroup of $G$.

The proof of Theorem 2.7 will last up to the end of this paper.

\section{Conditional measures}

The aim of the first four sections of this chapter is to study a non-invertible fibered dynamical system with an infinite volume fiber. We study it through its restriction to a well-chosen subset of finite volume in the fiber. The main output is 
Lemma 3.6, which gives a very simple formula for the conditional expectation with respect to the restriction of the $\sigma$-algebras of the future events. It will be used in section 8.1

In Section 3.5, we introduce a useful tool for studying probability measures which are invariant by a Markov operator $P$ when this Markov operator commutes with a group $L$. This tool will be used in sections 7.3 and 7.4 .

\subsection{Restriction and conditional expectation.}

We prove in this section a simple formula relating conditional expectation and restriction of $\sigma$-algebras.

Lemma 3.1. Let $(X, \mathcal{B}, \beta)$ be a $\sigma$-finite Lebesgue measure space and $\mathcal{A} \subset \mathcal{B}$ be a $\sigma$-finite $\sigma$-subalgebra. Let $i: Y \hookrightarrow X$ be a $\mathcal{B}$-measurable subset of $X$ with finite non-zero measure, $i^{-1} \mathcal{A}$ be the restriction of $\mathcal{A}$ to $Y$, and $\beta_{Y}:=\left.\frac{1}{\beta(Y)} \beta\right|_{Y}$.

Then, for any non-negative $\mathcal{B}$-measurable function $\varphi$ on $X$, for $\beta_{Y}$-almost every $x \in Y$, one has $\mathbb{E}\left(\mathbf{1}_{Y} \mid \mathcal{A}\right)(x) \neq 0$ and

$$
\mathbb{E}\left(\varphi \circ i \mid i^{-1} \mathcal{A}\right)(x)=\frac{\mathbb{E}\left(\varphi \mathbf{1}_{Y} \mid \mathcal{A}\right)(x)}{\mathbb{E}\left(\mathbf{1}_{Y} \mid \mathcal{A}\right)(x)} .
$$

Note that every $i^{-1} \mathcal{A}$-measurable function $\varphi_{0}$ on $Y$ can be expressed as $\varphi_{0}=\varphi \circ i$, where $\varphi$ is some $\mathcal{A}$-measurable function on $X$.

Proof. We first check that the denominator is non-zero for $\beta_{Y^{-}}$-almost every $y \in$ $Y$. We want to prove that the set $Z:=\left\{x \in X \mid \mathbb{E}\left(\mathbf{1}_{Y} \mid \mathcal{A}\right)(x)=0\right\}$ satisfies $\beta(Z \cap Y)=0$. But $Z$ is a $\mathcal{A}$-measurable subset of $X$, hence one has $\beta(Z \cap Y)=$ $\int_{Z} \mathbb{E}\left(\mathbf{1}_{Y} \mid \mathcal{A}\right)(x) \mathrm{d} \beta(x)=0$.

We now check equality (3.1). We introduce the $\mathcal{A}$-measurable function $\varphi^{\prime}$ on $X$ equal to the right-hand side of (3.1) when the denominator is non-zero and equal to zero if the denominator is zero. We want to prove, for $\beta_{Y}$-almost every $x \in Y$, the equality $\mathbb{E}\left(\varphi \circ i \mid i^{-1} \mathcal{A}\right)(x)=\varphi^{\prime}(x)$. To this aim, we have to check the equality

$$
\int_{Y} \varphi \psi \mathrm{d} \beta_{Y}=\int_{Y} \varphi^{\prime} \psi \mathrm{d} \beta_{Y}
$$

for every non-negative $\mathcal{A}$-measurable function $\psi$ on $X$. We compute

$$
\int_{X} \varphi \mathbf{1}_{Y} \psi \mathrm{d} \beta=\int_{X} \varphi^{\prime} \mathbb{E}\left(\mathbf{1}_{Y} \mid \mathcal{A}\right) \psi \mathrm{d} \beta=\int_{X} \varphi^{\prime} \mathbf{1}_{Y} \psi \mathrm{d} \beta
$$

and get the required equality by dividing by $\beta(Y)$.

\subsection{A fibered dynamical system.}

In this section, we prove a conditional expectation formula for a fibered dynamical system whose fiber has infinite volume.

Let $(B, \mathcal{B})$ be a standard Borel space, i.e. isomorphic as a Borel space to an interval, $\beta$ be a positive $\sigma$-finite Borel measure on $B$ and $T$ be a measurable endomorphism of $B$ preserving $\beta$. Let $(X, \mathcal{X})$ be a standard Borel space, $\mathcal{M}(X)$ the space of positive Borel measures on $X, B^{X}$ the product $B \times X$ endowed with the product $\sigma$-algebra $\mathcal{B}^{X}=\mathcal{B} \otimes \mathcal{X}, \pi: B \times X \rightarrow B$ the projection on the first factor and $T^{X}$ a measurable transformation of $B^{X}$ such that $\pi \circ T^{X}=T \circ \pi$. Let us write, for $(b, x)$ in $B \times X, T^{X}(b, x)=(T b, \rho(b) x)$.

Let $b \mapsto \nu_{b} \in \mathcal{M}(X)$ be a family of positive $\sigma$-finite measures on $X$. We assume that this family is measurable in the following sense: there exists an increasing 
sequence $\left(X_{n}\right)$ of Borel subsets of $X$ such that $X=\bigcup_{n} X_{n}$ and that, for any $n$, one has $\nu_{b}\left(X_{n}\right)<\infty$ for $\beta$-almost any $b$ and the map $\left.b \mapsto \nu_{b}\right|_{X_{n}}$ is a Borel map from $B$ to the space of finite Borel measures on $X_{n}$, equipped with its natural Borel structure.

Suppose, for $\beta$-almost every $b$ in $B$, one has $\nu_{T b}=\rho(b)_{*} \nu_{b}$. Set $\beta^{X}$ to be the Borel measure on $B^{X}$ given by $\beta^{X}=\int_{B} \delta_{b} \otimes \nu_{b} \mathrm{~d} \beta(b)$. This positive measure $\beta^{X}$ is $T^{X}$-invariant (this follows from the same computation as in Lemma 2.4 of [4]).

Various fibered dynamical systems $\left(B^{X}, \mathcal{B}^{X}, \beta^{X}, T^{X}\right)$ will play an important role in this paper.

For $\beta$-almost every $b$ in $B$, let us denote by $\beta_{b}^{T^{-1} \mathcal{B}}$ the conditional probability measure at $b$ of $\beta$ with respect to $T^{-1} \mathcal{B}$ so that, for every $\mathcal{B}$-measurable and $\beta^{X}$ integrable function $\psi: B \rightarrow \mathbb{C}$ and for $\beta$-almost every $b$ in $B$, one has

$$
\mathbb{E}\left(\psi \mid T^{-1} \mathcal{B}\right)(b)=\int_{B} \psi\left(b^{\prime}\right) \mathrm{d} \beta_{b}^{T^{-1}} \mathcal{B}\left(b^{\prime}\right) .
$$

The following lemma improves Lemma 2.5 of [4].

Lemma 3.2. Let us assume that for $\beta$-almost every $b$ in $B$ the map $\rho(b): X \rightarrow X$ is an automorphism of $X$. Then, for every $\mathcal{B}^{X}$-measurable and $\beta^{X}$-integrable function $\varphi: B^{X} \rightarrow \mathbb{C}$ and for $\beta^{X}$-almost every $(b, x)$ in $B^{X}$, one has the equality

$$
\mathbb{E}\left(\varphi \mid\left(T^{X}\right)^{-1}\left(\mathcal{B}^{X}\right)\right)(b, x)=\int_{B} \varphi\left(b^{\prime}, \rho\left(b^{\prime}\right)^{-1} \rho(b) x\right) \mathrm{d} \beta_{b}^{T^{-1} \mathcal{B}}\left(b^{\prime}\right) .
$$

Proof. The only difference with Lemma 2.5 of 4 is that in Lemma 3.2 the positive measures $\beta$ and $\nu_{b}$ are not assumed to be finite. The proof is given by the same computation.

\subsection{A conditional expectation formula.}

In this section we apply the simple formula (3.1) to extensions of Bernoulli dynamical systems.

Let $(A, \mathcal{A}, \alpha)$ be a Lebesgue probability space and $(B, \mathcal{B}, \beta, T)$ be the one-sided Bernoulli shift with alphabet $(A, \mathcal{A}, \alpha)$; that is, $B$ is the product space $B=A^{\mathbb{N}}, \mathcal{B}$ is the product $\sigma$-algebra, $\beta$ is the product probability measure $\beta=\alpha^{\otimes \mathbb{N}}$ and $T$ is the shift given by $T\left(b_{0}, b_{1}, \ldots\right)=\left(b_{1}, b_{2}, \ldots\right)$.

Let $Z$ be a second-countable locally compact topological group with a given left Haar measure $\lambda_{Z}$ and $\theta: B \rightarrow Z$ a measurable map. We introduce the extension

$$
\left(B^{\theta}, \mathcal{B}^{\theta}, \beta^{\theta}, T^{\theta}\right) \text {. }
$$

The space $B^{\theta}$ is the product $B \times Z$, the $\sigma$-algebra $\mathcal{B}^{\theta}$ is the product $\sigma$-algebra, the measure $\beta^{\theta}$ is the product $\beta \otimes \lambda_{Z}$ and the transformation $T^{\theta}$ is given by, for $(b, z) \in B^{\theta}$,

$$
T^{\theta}(b, z)=\left(T b, \theta(b)^{-1} z\right) .
$$

As soon as $Z$ is not compact, the measure $\beta^{\theta}$ has infinite volume. The transformation $T^{\theta}$ preserves the measure $\beta^{\theta}$.

For $n \geq 0$, let $\theta_{n}: B \rightarrow Z$ be the function given by, for $b \in B$,

$$
\theta_{n}(b)=\theta(b) \theta(T b) \cdots \theta\left(T^{n-1} b\right) .
$$

Let $\mathcal{Q}_{n}^{\theta}$ be the $\sigma$-subalgebra $\mathcal{Q}_{n}^{\theta}:=\left(T^{\theta}\right)^{-n}\left(\mathcal{B}^{\theta}\right)$. The drift argument in section 8.1 is based on a formula for the conditional expectation with respect to this $\sigma$-algebra. Note that the intersection $\mathcal{Q}_{\infty}^{\theta}:=\bigcap_{n \geq 0} \mathcal{Q}_{n}^{\theta}$ is the tail $\sigma$-algebra. 
For $a=\left(a_{0}, \ldots, a_{n-1}\right) \in A^{n}$ and $b \in B$ we set

$$
h_{n, b}(a)=a T^{n} b:=\left(a_{0}, a_{1}, \ldots, a_{n-1}, b_{n}, b_{n+1}, \ldots\right) .
$$

As $a$ varies, these elements parametrize the fiber $T^{-n}\left(T^{n} b\right) \subset B$. For $\beta$-almost every $b$ in $B$, the conditional probability measures $\beta_{n, b}$ of $\beta$ along $T^{-n} \mathcal{B}$ are the images

$$
\beta_{n, b}=\left(h_{n, b}\right)_{*} \alpha^{\otimes n}
$$

For $c=(b, z) \in B^{\theta}$, we set

$$
h_{n, c}(a)=\left(a T^{n} b, \theta_{n}\left(a T^{n} b\right) \theta_{n}(b)^{-1} z\right) .
$$

As $a$ varies, these elements parametrize the fiber $\left(T^{\theta}\right)^{-n}\left(\left(T^{\theta}\right)^{n} c\right) \subset B^{\theta}$. Lemma 3.3 below tells us that, for $\beta^{\theta}$-almost every $c \in B^{\theta}$, the conditional probability measures $\beta_{n, c}^{\theta}$ of $\beta^{\theta}$ along $\mathcal{Q}_{n}^{\theta}$ are the images

$$
\beta_{n, c}^{\theta}=\left(h_{n, c}\right)_{*} \alpha^{\otimes n} .
$$

Lemma 3.3. Let $n$ be a positive integer. The conditional expectation with respect to the $\sigma$-algebra $\mathcal{Q}_{n}^{\theta}$ is given by, for every non-negative $\mathcal{B}^{\theta}$-measurable function $\varphi$ and for $\beta^{\theta}$-almost every $c=(b, z)$ in $B^{\theta}$,

$$
\mathbb{E}\left(\varphi \mid \mathcal{Q}_{n}^{\theta}\right)(c)=\int_{A^{n}} \varphi\left(h_{n, c}(a)\right) \mathrm{d} \alpha^{\otimes n}(a) .
$$

Proof. This follows from formula (3.3) and Lemma 3.2 with $X=Z$ and with the constant family of measures $b \mapsto \lambda_{Z}$ on $Z$.

Since the measure $\beta^{\theta}$ has infinite volume, it will be convenient to introduce its restriction to a subset of finite volume. Let $U$ be a Borel subset of $Z$ with $0<$ $\lambda_{Z}(U)<\infty$. We introduce the probability space $\left(B^{U}, \mathcal{B}^{U}, \beta^{U}\right)$, where $B^{U}:=B \times U$, $\mathcal{B}^{U}$ is the product of $\mathcal{B}$ with the Borel $\sigma$-algebra of $U$ and $\beta^{U}$ is the probability on $B \times U$ given by $\beta^{U}=\left.\frac{1}{\lambda_{Z}(U)}\left(\beta \otimes \lambda_{Z}\right)\right|_{B^{U}}$. Let $\mathcal{Q}_{n}^{U}$ be the sub- $\sigma$-algebra of $\mathcal{B}^{U}$ which is the restriction of $\mathcal{Q}_{n}^{\theta}$ to $B^{U}$. It is the sub- $\sigma$-algebra of $\mathcal{B}^{U}$ generated by the maps $(b, u) \mapsto T^{n} b$ and $(b, u) \mapsto \theta_{n}(b)^{-1} u$. For $\beta^{U}$-almost every $c=(b, z)$ in $B \times U$, let $\beta_{n, c}^{U}$ be the conditional probability measure of $\beta^{U}$ at $c=(b, z) \in B \times U$ along $\mathcal{Q}_{n}^{U}$ and set

$$
Q_{n, c}^{U}:=h_{n, c}^{-1}\left(B^{U}\right)=\left\{a \in A^{n} \mid \theta_{n}\left(a T^{n} b\right) \theta_{n}(b)^{-1} z \in U\right\} .
$$

Let $\varphi$ be a non-negative $\mathcal{B}^{\theta}$-measurable function on $B^{\theta}$ and denote by $\varphi_{U}$ the restriction of $\varphi$ to $B^{U}$. By definition, for $\beta^{U}$-almost every $c$ in $B^{U}$, one has

$$
\mathbb{E}\left(\varphi_{U} \mid \mathcal{Q}_{n}^{U}\right)(c)=\int_{B^{U}} \varphi_{U}\left(c^{\prime}\right) \mathrm{d} \beta_{n, c}^{U}\left(c^{\prime}\right)
$$

We introduce the notation

$$
\alpha^{\otimes n}\left(E_{1} \mid E_{2}\right)=\frac{\alpha^{\otimes n}\left(E_{1} \cap E_{2}\right)}{\alpha^{\otimes n}\left(E_{2}\right)}
$$

for the conditional probability of two measurable subsets $E_{1}, E_{2}$ of $A^{n}$. 
Lemma 3.4. The conditional expectation with respect to the $\sigma$-algebra $\mathcal{Q}_{n}^{U}$ is given by, for every non-negative $\mathcal{B}^{\theta}$-measurable function $\varphi$ and for $\beta^{U}$-almost every $c=$ $(b, z)$ in $B^{U}$,

$$
\mathbb{E}\left(\varphi_{U} \mid \mathcal{Q}_{n}^{U}\right)(c)=\frac{\int_{Q_{n, c}^{U}} \varphi\left(h_{n, c}(a)\right) \mathrm{d} \alpha^{\otimes n}(a)}{\alpha^{\otimes n}\left(Q_{n, c}^{U}\right)} .
$$

Hence, in other words, for every $\mathcal{B}^{\theta}$-measurable subset $K \subset B^{U}$, for $\beta^{U}$-almost every $c=(b, z)$ in $B^{U}$,

$$
\beta_{n, c}^{U}(K)=\mathbb{E}\left(\mathbf{1}_{K} \mid \mathcal{Q}_{n}^{U}\right)(c)=\alpha^{\otimes n}\left(h_{n, c}^{-1}(K) \mid Q_{n, c}^{U}\right) .
$$

Proof. We first note that Lemmas 3.1 and 3.3 tell us that the denominator on the right-hand side of (3.7) is non-zero for $\beta^{U}$-almost every $c \in B^{U}$. These formulae also follow from Lemmas 3.1 and 3.3 .

\subsection{Conditional measure, fibration and restriction.}

In this section we mix together the results of sections 3.2 and 3.3 .

We keep the notation and hypothesis of section 3.3 which are related to the dynamical system $\left(B^{\theta}, \mathcal{B}^{\theta}, \beta^{\theta}, T^{\theta}\right)$ and its restriction to $B \times U$. We keep also the data of section 3.2 the space $(X, \mathcal{X})$ is a standard Borel space, endowed with a $\mathcal{B}$-measurable family of positive measures $b \mapsto \nu_{b} ; B \rightarrow \mathcal{M}(X)$ such that, for $\beta$ almost every $b$ in $B$, one has $\nu_{T b}=\rho(b)_{*} \nu_{b}$. These data allow us to construct the dynamical system $\left(B^{X}, \mathcal{B}^{X}, \beta^{X}, T^{X}\right)$.

We introduce the dynamical system $\left(B^{\theta, X}, \mathcal{B}^{\theta, X}, \beta^{\theta, X}, T^{\theta, X}\right)$, where the space $B^{\theta, X}$ is the product $B^{\theta} \times X$, the $\sigma$-algebra $\mathcal{B}^{\theta, X}$ is the product $\sigma$-algebra, the measure $\beta^{\theta, X}$ is given by

$$
\beta^{\theta, X}=\int_{B^{\theta}} \delta_{(b, z)} \otimes \nu_{b} \mathrm{~d} \beta^{\theta}(b, z)
$$

and the transformation $T^{\theta, X}$ is given by, for $(c, x)=(b, z, x) \in B^{\theta, X}$,

$$
T^{\theta, X}(b, z, x)=\left(T b, \theta(b)^{-1} z, \rho(b) x\right) .
$$

As soon as $Z$ is not compact, this measure $\beta^{\theta, X}$ has infinite volume. The transformation $T^{\theta, X}$ preserves the measure $\beta^{\theta, X}$.

Let $\mathcal{Q}_{n}^{\theta, X}$ be the sub- $\sigma$-algebra

$$
\mathcal{Q}_{n}^{\theta, X}:=\left(T^{\theta, X}\right)^{-n}\left(\mathcal{B}^{\theta, X}\right) .
$$

Note again that the intersection $\mathcal{Q}_{\infty}^{\theta, X}:=\bigcap_{n \geq 0} \mathcal{Q}_{n}^{\theta, X}$ is the tail $\sigma$-algebra.

For $a=\left(a_{0}, \ldots, a_{n-1}\right) \in A^{n}$ and $(c, x)=(b, z, x) \in B^{\theta, X}$, we set

$$
h_{n, c, x}(a)=\left(a T^{n} b, \theta_{n}\left(a T^{n} b\right) \theta_{n}(b)^{-1} z, \rho_{n}\left(a T^{n} b\right)^{-1} \rho_{n}(b) x\right),
$$

where $\rho_{n}(b)=\rho\left(T^{n-1} b\right) \cdots \rho(b)$. As $a$ varies, these elements parametrize the fiber

$$
\left(T^{\theta, X}\right)^{-n}\left(\left(T^{\theta, X}\right)^{n}(c, x)\right) \subset B^{\theta, X} .
$$

The following lemma tells us that, for $\beta^{\theta, X}$-almost every $(c, x) \in B^{\theta, X}$, the conditional probability measures $\beta_{n, c, x}^{\theta, X}$ of $\beta^{\theta, X}$ along $\mathcal{Q}_{n}^{\theta, X}$ are the images

$$
\beta_{n, c, x}^{\theta, X}=\left(h_{n, c}\right)_{*} \alpha^{\otimes n} .
$$


Lemma 3.5. The conditional expectation with respect to the $\sigma$-algebra $\mathcal{Q}_{n}^{\theta, X}$ is given by, for every non-negative $\mathcal{B}^{\theta, X}$-measurable function $\varphi$ and for $\beta^{\theta, X}$-almost every $(c, x)$ in $B^{\theta, X}$,

$$
\mathbb{E}\left(\varphi \mid \mathcal{Q}_{n}^{\theta, X}\right)(c, x)=\int_{A^{n}} \varphi\left(h_{n, c, x}(a)\right) \mathrm{d} \alpha^{\otimes n}(a) .
$$

Proof. This is a consequence of Lemmas 3.2 and 3.3 .

Again let $U$ be a Borel subset of $Z$ with $\lambda_{Z}(U)<\infty$. We introduce the probability space

$$
\left(B^{U, X}, \mathcal{B}^{U, X}, \beta^{U, X}\right)
$$

where $B^{U, X}:=B^{U} \times X, \mathcal{B}^{U, X}:=\mathcal{B}^{U} \otimes \mathcal{X}$ and $\beta^{U, X}$ is the probability measure on $B^{U, X}$ given by $\beta^{U, X}=\int_{B^{U}} \delta_{(b, z)} \otimes \nu_{b} \mathrm{~d} \beta^{U}(b, z)$. Let $\mathcal{Q}_{n}^{U, X}$ be the sub- $\sigma$-algebra of $\mathcal{B}^{U, X}$ which is the restriction of $\mathcal{Q}_{n}^{\theta, X}$ to $B^{U, X}$.

The drift argument in section 8.1 is based on a formula for the conditional expectation with respect to this $\sigma$-algebra: formula (3.14).

For $\beta^{U, X}$-almost every $(c, x)$ in $B^{U, X}$, let $\beta_{n, c, x}^{U, X}$ be the conditional probability measure of $\beta^{U, X}$ at $(c, x)$ along $\mathcal{Q}_{n}^{U, X}$. Let $\varphi$ be a non-negative $\mathcal{B}^{\theta, X}$-measurable function on $B^{\theta, X}$ and denote by $\varphi_{U}$ the restriction of $\varphi$ to $B^{U, X}$. By definition, for $\beta^{U, X}$-almost every $(c, x)$ in $B^{U, X}$, one has

$$
\mathbb{E}\left(\varphi_{U} \mid \mathcal{Q}_{n}^{U, X}\right)(c, x)=\int_{B^{U} \times X} \varphi\left(c^{\prime}, x^{\prime}\right) \mathrm{d} \beta_{n, c, x}^{U, X}\left(c^{\prime}, x^{\prime}\right) .
$$

Lemma 3.6. The conditional expectation with respect to the $\sigma$-algebra $\mathcal{Q}_{n}^{U, X}$ is given by, for every non-negative $\mathcal{B}^{\theta, X}$-measurable function $\varphi$ and for $\beta^{U, X}$-almost every $(c, x)$ in $B^{U} \times X$,

$$
\mathbb{E}\left(\varphi_{U} \mid \mathcal{Q}_{n}^{U, X}\right)(c)=\frac{\int_{Q_{n, c}^{U}} \varphi\left(h_{n, c, x}(a)\right) \mathrm{d} \alpha^{\otimes n}(a)}{\alpha^{\otimes n}\left(Q_{n, c}^{U}\right)} .
$$

Hence, in other words, for every $\mathcal{B}^{\theta}$-measurable subset $K \subset B^{U} \times X$ and for $\beta^{U, X}$ almost every $(c, x)$ in $B^{U} \times X$, one has

$$
\beta_{n, c, x}^{U, X}(K)=\mathbb{E}\left(\mathbf{1}_{K} \mid \mathcal{Q}_{n}^{U, X}\right)(c, x)=\alpha^{\otimes n}\left(h_{n, c, x}^{-1}(K) \mid Q_{n, c}^{U}\right) .
$$

We recall that the set $Q_{n, c}^{U}$ has been defined in (3.5) and that the notation for the conditional probability measure has been introduced in (3.6).

Proof. This formula follows from Lemmas 3.1 and 3.5 .

\subsection{Conditional measure along the centralizer.}

In this section we investigate the relationship between probability measures $\nu$, which are invariant and ergodic for some Markov operator $P$, and the orbits of a group $L$ of transformations commuting with $P$.

Let $(X, \mathcal{X})$ be a standard Borel space and $P$ be a Markov operator on $X$, i.e. a measurable family $X \rightarrow \mathcal{P}(X) ; x \mapsto P_{x}$ of Borel probability measures on $X$. It induces a continuous linear map also denoted by $P: \mathcal{L}^{\infty}(X, \mathcal{X}) \rightarrow \mathcal{L}^{\infty}(X, \mathcal{X}) ; \varphi \mapsto$ $P \varphi$ where, for $x \in X,(P \varphi)(x)=\int_{X} \varphi(y) \mathrm{d} P_{x}(y)$. Suppose $X$ is endowed with a Borel action of a locally compact second countable group $L$ and this action commutes with $P$ (i.e. one has $P_{\ell x}=\ell_{*} P_{x}$ for any $\ell$ in $L$ and $x$ in $X$ ), that is, 
$P(\varphi \circ \ell)=P \varphi \circ \ell$ for any $\varphi$ in $\mathcal{L}^{\infty}(X, \mathcal{X})$ and $\ell$ in $L$. Let $\nu$ be a Borel probability measure on $X$ which is $P$-invariant and $P$-ergodic, that is, for every $\varphi$ in $\mathcal{L}^{\infty}(X, \mathcal{X})$, $\int_{X} P \varphi \mathrm{d} \nu=\int_{X} \varphi \mathrm{d} \nu$ and, if $P \varphi=\varphi$, then $\varphi$ is $\nu$-almost surely constant.

Lemma 3.7. Let $L_{\nu}:=\left\{\ell \in L \mid \ell_{*} \nu=\nu\right\}$ be the stabilizer of $\nu$ in $L$. There exists a Borel subset $E$ of $X$ such that $\nu(E)=1, E$ is $L_{\nu}$-invariant and, for all $\ell \in L \backslash L_{\nu}$, one has $\ell E \cap E=\emptyset$.

Remark 3.8. Assume $L$ is unimodular and its action on $X$ has discrete stabilizers and denote, for $\nu$-almost every $x$ in $X$, by $\sigma^{L}(x)$ the conditional measure at $x$ of $\nu$ along the action of $L$ as in [4, Sect. 4.1]. The lemma implies that, for $\nu$-almost any $x$ in $X, \sigma^{L}(x)$ is a Haar measure on $L$.

Proof. According to Varadarajan's theorem (cf. 28]), one can assume that $X$ is a compact metric space and that the action of $L$ on $X$ is continuous. Let us denote by $C^{0}(X)$ the set of continuous functions on $X$ and by $E$ the set of generic points for $\nu$, i.e.

$$
E:=\left\{x \in X \mid \forall \varphi \in C^{0}(X), \frac{1}{n} \sum_{0 \leq k<n} P^{k} \varphi(x) \underset{n \rightarrow \infty}{\longrightarrow} \int_{X} \varphi \mathrm{d} \nu\right\} .
$$

Since $\nu$ is ergodic and since $C^{0}(X)$ contains a countable dense subset, according to Chacon-Ornstein's ergodic theorem, one has $\nu(E)=1$. Since $\nu$ is $L_{\nu}$-invariant and since the action of $L$ commutes with $P$, the set $E$ is also $L_{\nu}$-invariant. Conversely, if $\ell$ is in $L \backslash L_{\nu}$, then $\ell E$ is the set of generic points for the $P$-invariant and $P$-ergodic probability measure $\ell_{*} \nu$. Since $\ell_{*} \nu \neq \nu$, one has $\ell E \cap E=\emptyset$.

\section{RANDOM WALKS ON SEMISIMPLE GROUPS}

In this chapter we develop new crucial properties of random walks on a product $H$ of real and $p$-adic semisimple groups. We will apply them in chapters 5 , 7 and 8 to the Zariski closure of the adjoint group $\operatorname{Ad} \Gamma_{\mu}$. The reader may concentrate at first glance on the case where $H$ is a real semisimple group. We will choose notation very similar to the standard notation in this case.

In sections 4.1, 4.2 and 4.3 we recall basic notation and facts on semisimple algebraic groups, their random walks, their flag varieties and their representations.

In sections 4.4 and 4.5 we recall from 9. asymptotic properties of these random walks: simplicity of the Lyapounov, central limit theorem, law of the iterated logarithm, local limit theorem, large deviation principle, spectral gap of the transfer operator, and Hölder regularity of the Furstenberg stationary measure.

All these properties are used in sections 4.6 and 4.7 to prove the "law of the angles" (Theorem 4.19). In section 4.8 we deduce from the law of the angles a control (Proposition 4.21) of the norm and the direction of some vector that will be the "drift vector" later on in Lemma 8.2 .

\subsection{Products of semisimple $\mathbb{Q}_{p}$-groups.}

We first recall some basic facts on real and $p$-adic semisimple algebraic groups. We refer to [27] for a more detailed account of the structure theory in the $p$-adic case.

Let $\mathbb{Q}_{p}$ be the field of $p$-adic numbers when $p$ is a prime number and let $\mathbb{Q}_{\infty}$ be the field of real numbers. Let $\{\infty, 2,3,5, \ldots\}$ be the set of prime numbers including $\infty$ and $\mathcal{S}$ be a finite subset of it. We let $\mathbb{Q}_{\mathcal{S}}$ be the locally compact algebra $\bigoplus_{p \in \mathcal{S}} \mathbb{Q}_{p}$. 
For each $p$ in $\mathcal{S}$, let $\mathbf{H}_{p}$ be a connected semisimple algebraic group defined over $\mathbb{Q}_{p}$ and $H_{p}$ be the group of $\mathbb{Q}_{p}$-points of $\mathbf{H}_{p}$. Let $H=\prod_{p \in \mathcal{S}} H_{p}$ and $\mathcal{H}$ be the Borel $\sigma$-algebra of $H$.

By definition, a minimal parabolic subgroup $P$ of $H$ is the product $P=\prod_{p \in \mathcal{S}} P_{p}$ of the groups $P_{p}$ of $\mathbb{Q}_{p}$-points of minimal parabolic subgroups of $\mathbf{H}_{p}$. Similarly, a maximal split subtorus $A$ of $H$ is the product $A=\prod_{p \in \mathcal{S}} A_{p}$ of the groups $A_{p}$ of $\mathbb{Q}_{p}$-points of maximal $\mathbb{Q}_{p}$-split subtori of $\mathbf{H}_{p}$, and so on, allowing us to use the language of semisimple algebraic groups in this product situation. We fix such $A$ and $P$ with $A \subset P$ and let $U=\prod U_{p}$ be the unipotent radical of $P$. We denote by $\Sigma$ the set of restricted roots of $A$ in the Lie algebra of $H$ and by $\Pi \subset \Sigma$ the set of restricted simple roots associated to $P$. The group $U$ is spanned by the unipotent one-parameter subgroups of $H$ whose Lie algebras are contained in the union of the weight spaces associated to the elements of $\Pi$. The homogeneous space $\mathcal{P}_{\Pi}:=H / P$ is called the full flag variety.

For $\Theta \subset \Pi$, we let $P_{\Theta}$ be the standard parabolic subgroup of $H$ associated to $\Theta$, that is, the subgroup of $H$ spanned by $P=P_{\Pi}$ and the one-parameter unipotent subgroups of $H$ whose Lie algebras are contained in the union of the weight spaces associated to the $-\alpha, \alpha \in \Pi \backslash \Theta$. We let $U_{\Theta}$ denote the unipotent radical of $P_{\Theta}$, that is, the group spanned by the one-parameter unipotent subgroups of $H$ whose Lie algebras are contained in the weight spaces associated to the positive roots $\alpha$ which may not be written as linear combinations of the elements of $\Pi \backslash \Theta$. We set $\mathcal{P}_{\Theta}=H / P_{\Theta}$ and let $\eta_{\Theta}$ denote the fixed point of $P_{\Theta}$ in $\mathcal{P}_{\Theta}$.

Let $Z=\prod Z_{p}$ be the centralizer of $A$ so that $P=Z U$ and $Z / A$ is compact. Any continuous homomorphism $A \rightarrow \mathbb{R}$ extends in a unique way as a continuous homomorphism $Z \rightarrow \mathbb{R}$. More precisely, let $\mathfrak{a}$ be the dual space of the real vector space of continuous homomorphisms $A \rightarrow \mathbb{R}$ : the dimension of $\mathfrak{a}$ is the sum of the relative ranks of the groups $\mathbf{H}_{p}, p \in \mathcal{S}$, and there exists a unique continuous morphism $\omega: Z \rightarrow \mathfrak{a}$ whose restriction to $A$ is the natural morphism $A \rightarrow \mathfrak{a}$. If, for some $p$ in $\mathcal{S}, \chi: A_{p} \rightarrow \mathbb{Q}_{p}^{*}$ is a rational character, we let $\chi^{\omega}$ be the unique linear form on $\mathfrak{a}$ such that, for any $a$ in $A, \chi^{\omega}(\omega(a))=\log \left|\chi\left(a_{p}\right)\right|$, where $a_{p}$ is the $p^{\text {th }}$ component of $a$. We can see $\Sigma^{\omega}$ as a root system on $\mathfrak{a}$ and $\Pi^{\omega}$ as a set of simple roots for $\Sigma^{\omega}$. We let $\mathfrak{a}^{+}$(resp. $\mathfrak{a}^{++}$) denote the Weyl chamber (resp. the open Weyl chamber) associated to $\Pi^{\omega}$, and we set $Z^{+}=\omega^{-1}\left(\mathfrak{a}^{+}\right)\left(\right.$resp. $\left.Z^{++}=\omega^{-1}\left(\mathfrak{a}^{++}\right)\right)$.

For $\Theta \subset \Pi$, we let $Z_{\Theta}$ be the reductive subgroup of $P_{\Theta}$ that contains $A$ and we set $A_{\Theta}=\bigcap_{\alpha \in \Pi \backslash \Theta} \operatorname{ker} \alpha$ and $\mathfrak{a}_{\Theta}=\bigcap_{\alpha \in \Pi \backslash \Theta} \operatorname{ker} \alpha^{\omega}$. One has $P_{\Theta}=Z_{\Theta} U_{\Theta}$. Again, the natural morphism $A_{\Theta} \rightarrow \mathfrak{a}_{\Theta}$ extends in a unique way as a morphism $\omega_{\Theta}: Z_{\Theta} \rightarrow \mathfrak{a}_{\Theta}$.

Let $K=\prod K_{p}$ be a good maximal compact subgroup of $H$, where good means that the image in $\operatorname{Aut}(A)$ of the normalizer of $A$ in $K$ contains the relative Weyl group. Such a $K$ always exists. One has the Cartan decomposition $H=K Z^{+} K$. For every element $h \in H$, we choose a decomposition $h=k_{h} z_{h} \ell_{h}$ with $k_{h} \in K$, $z_{h} \in Z^{+}$and $\ell_{h} \in K$, even though this decomposition is not unique. We introduce the Cartan projection $\kappa: H \rightarrow \mathfrak{a} ; h \mapsto \kappa(h):=\omega\left(z_{h}\right)$.

\subsection{Random walks on products of semisimple $\mathbb{Q}_{p}$-groups.}

We now recall the construction of Furstenberg's boundary map for products of independent identically distributed elements of $H$. 
Let $\mu$ be a Borel probability measure on $H, \Gamma_{\mu}^{+}$be the smallest closed subsemigroup of $H$ containing the support of $\mu$ and $\Gamma_{\mu}$ be the smallest closed subgroup of $H$ containing the support of $\mu$. We assume that, for all $p$, the projection $\Gamma_{\mu, p}$ of $\Gamma_{\mu}$ in $H_{p}$ is Zariski dense in $H_{p}$. We let $\check{\mu}$ be the probability measure on $H$ which is the image of $\mu$ by the map $h \mapsto h^{-1}$.

We do not assume in this chapter that $\mu$ has compact support, but from section 4.4 on, we will assume that $\mu$ has finite first moment and from Proposition 4.5 on, that $\mu$ has finite exponential moments. All these integrability conditions are satisfied when $\mu$ has compact support.

The main difference between the real case and the non-archimedean one is that, when $p$ is finite, the action of $\Gamma_{\mu, p}$ on the full flag variety $H_{p} / P_{p}$ might be nonproximal. We set

$$
\Theta_{\mu}:=\left\{\alpha \in \Pi \mid \alpha^{\omega}\left(\kappa\left(\Gamma_{\mu}^{+}\right)\right) \text {is unbounded }\right\} .
$$

The set $\Theta_{\check{\mu}}$ is then the image of $\Theta_{\mu}$ by the opposition involution $\iota$, that is, the opposite of the unique element of the Weyl group of $\Sigma^{\omega}$ that sends $\mathfrak{a}^{+}$to $-\mathfrak{a}^{+}$. We write $P_{\mu}=P_{\Theta_{\mu}}, \mathcal{P}_{\mu}=\mathcal{P}_{\Theta_{\mu}}, \mathfrak{a}_{\mu}=\mathfrak{a}_{\Theta_{\mu}}$, etc. We know from 2 that $\Theta_{\mu}$ is the largest subset of $\Pi$ such that the action of $\Gamma_{\mu}^{+}$on $\mathcal{P}_{\mu}=\mathcal{P}_{\Theta_{\mu}}$ is proximal and that, for any $\alpha$ in $\Pi \backslash \Theta_{\mu}$, one has $\sup _{h \in \Gamma_{\mu}^{+}} \alpha^{\omega}(\kappa(h))<\infty$. According to a result of Goldsheid and Margulis that we will not use, when $\mathcal{S}=\{\infty\}$, one has $\Theta_{\mu}=\Pi$. We denote by $\xi_{0}^{M}=\eta_{\Theta_{\mu}} \in \mathcal{P}_{\mu}$ the fixed point of $P_{\mu}$ in $\mathcal{P}_{\mu}$. This is the only attractive fixed point of the elements of $Z^{++}$in $\mathcal{P}_{\mu}$. We let $\mathcal{Q}_{0}^{m}$ be the complement of its attraction region. For $h \in H$ we set $\xi_{h}^{M}:=k_{h} \xi_{0}^{M}$ and $\mathcal{Q}_{h}^{m}:=\ell_{h}^{-1} \mathcal{Q}_{0}^{m}$. The set $\mathcal{Q}_{h}^{m}$ is a maximal Schubert variety, i.e. a maximal closed subset $\mathcal{Q} \varsubsetneqq \mathcal{P}_{\mu}$ which is invariant under some conjugate of $U$. The point $\xi_{h}^{M}$ is called the density point of $h$ in $\mathcal{P}_{\mu}$. Similarly, we denote by $\xi_{0}^{m} \in \mathcal{P}_{\breve{\mu}}$ the repulsive fixed point of all the elements of $Z^{++}$(that is, the fixed point of the parabolic subgroup opposite to $P_{\mu}$ with respect to $A$ ) and, for $h$ in $H$, we set $\xi_{h}^{m}:=\ell_{h}^{-1} \xi_{0}^{m}$ so that $\xi_{h}^{m}$ is the density point of $h^{-1}$ in $\mathcal{P}_{\breve{\mu}}$.

Let $(B, \mathcal{B}, \beta, T)$ be the one-sided Bernoulli shift whose alphabet is $(H, \mathcal{H}, \mu)$. We recall (see 9 for example) that there exists a unique $\mathcal{B}$-measurable map

$$
\xi: B \rightarrow \mathcal{P}_{\mu} ; b \mapsto \xi_{b} \text { such that } \xi_{b}=b_{0} \xi_{T b},
$$

for $\beta$-almost every $b$ in $B$. The image probability measure $\xi_{*} \beta$ is then the unique $\mu$ stationary probability measure $\nu_{\mu}$ on $\mathcal{P}_{\mu}$. For any proper algebraic subvariety $\mathcal{Q}$ of $\mathcal{P}_{\mu}$, one has $\nu_{\mu}(\mathcal{Q})=0$. In the same way, we let $\nu_{\check{\mu}}$ denote the unique $\check{\mu}$-stationary probability measure on $\mathcal{P}_{\check{\mu}}$.

Let $s: \mathcal{P}_{\mu} \rightarrow H / U_{\mu}$ be a Borel section of the projection $H / U_{\mu} \rightarrow \mathcal{P}_{\mu}$. The Iwasawa decomposition $H=K P_{\Pi}$ allows us to choose the section $s$ in such a way that, for every $k$ in $K$,

$$
s\left(k P_{\mu}\right)=k m(k) U_{\mu} \quad \text { with } m(k) \in Z_{\mu} \cap K .
$$

The group $Z_{\mu}$ acts simply and transitively by right multiplications on $P_{\mu} / U_{\mu}$. One denotes by $\sigma: H \times \mathcal{P}_{\mu} \rightarrow Z_{\mu}$ and $\sigma_{\mathbb{R}}: H \times \mathcal{P}_{\mu} \rightarrow \mathfrak{a}_{\mu}$ the Borel cocycles given by, for all $h$ in $H$ and all $\eta$ in $\mathcal{P}_{\mu}$,

$$
h s(\eta) \in s(h \eta) \sigma(h, \eta)
$$

and

$$
\sigma_{\mathbb{R}}(h, \eta)=\omega_{\mu}(\sigma(h, \eta))
$$


There exists a compact subset $L$ of $Z_{\mu}$ such that, for any $h$ in $\Gamma_{\mu}^{+}$and $\eta$ in the support of $\nu_{\mu}, \sigma(h, \eta)$ belongs to $L A_{\mu}=A_{\mu} L$.

By using the equivariant map $\xi$ and the cocycle $\sigma$, we can define natural maps $\theta: B \rightarrow Z_{\mu}, \theta_{\mathbb{R}}: B \rightarrow \mathfrak{a}_{\mu}$ and, for $n \geq 1, \theta_{n}: B \rightarrow Z_{\mu}, \theta_{\mathbb{R}, n}: B \rightarrow \mathfrak{a}_{\mu}$ as being the $\mathcal{B}$-measurable functions given by, for $\beta$-almost every $b$ in $B$,

$$
\begin{aligned}
\theta(b) & :=\sigma\left(b_{0}, \xi_{T b}\right)=\sigma\left(b_{0}^{-1}, \xi_{b}\right)^{-1}, \\
\theta_{n}(b) & :=\theta(b) \cdots \theta\left(T^{n-1} b\right)=\sigma\left(b_{0} \cdots b_{n-1}, \xi_{T^{n} b}\right), \\
\theta_{\mathbb{R}}(b) & :=\sigma_{\mathbb{R}}\left(b_{0}, \xi_{T b}\right) \\
\text { and } \theta_{\mathbb{R}, n}(b) & :=\sum_{0 \leq k<n} \theta_{\mathbb{R}}\left(T^{k} b\right)=\sigma_{\mathbb{R}}\left(b_{0} \cdots b_{n-1}, \xi_{T^{n} b}\right) .
\end{aligned}
$$

\subsection{Flag variety, representations and duality.}

In this section we explain the relation between the action of $\Gamma_{\mu}$ on the irreducible representations of $H$ and on the flag variety of $H$.

By a representation of $H$, we shall mean a finite product $V=\prod_{p \in \mathcal{S}} V_{p}$ of algebraic representations of $H_{p}$ in a $\mathbb{Q}_{p}$-vector space $V_{p}$. In this section we will only consider irreducible representations of $H$, i.e. the case where $V$ is equal to one of the $V_{p}$ and is an irreducible representation of $H_{p}$.

Let $p \in\{\infty, 2,3,5, \ldots\}$ and $V$ be a finite-dimensional $\mathbb{Q}_{p}$-vector space, endowed with a good norm, that is a euclidean norm if $p=\infty$ and a ultrametric one if $p$ is finite. We consider $V$ as a $\mathbb{Q}_{\mathcal{S}}$-module through the natural projection map $\mathbb{Q}_{\mathcal{S}} \rightarrow \mathbb{Q}_{p}$. We let $V^{*}$ denote the dual space of $V$ and, for any subset $W$ of $V^{*}, W^{\perp}$ denote its orthogonal in $V$. We also let $\operatorname{Gr}(V)$ denote the Grassmann variety of subspaces of $V$. If $V=V_{1} \oplus \cdots \oplus V_{r}$ is a decomposition of $V$ into a direct sum of subspaces, we say that this decomposition is good, when $p=\infty$, if it is an orthogonal decomposition and, when $p$ is finite, if for any $v_{1}, \ldots, v_{r}$ in $V_{1}, \ldots, V_{r}$, one has $\left\|v_{1}+\cdots+v_{r}\right\|=\max _{1 \leq i \leq r}\left\|v_{i}\right\|$. There exists a unique good norm on $\bigwedge^{2} V$ such that, for any good direct sum $V_{1} \oplus V_{2} \subset V$, the direct sum $\bigwedge^{2} V_{1} \oplus\left(V_{1} \wedge V_{2}\right) \oplus \bigwedge^{2} V_{2}$ is good and, for any $v_{1} \in V_{1}$ and $v_{2} \in V_{2}$, one has $\left\|v_{1} \wedge v_{2}\right\|=\left\|v_{1}\right\|\left\|v_{2}\right\|$. The distance associated to the norm on the projective space $\mathbb{P}(V)$ is then given by, for two lines $x_{1}, x_{2}$ in $\mathbb{P}(V)$ generated respectively by $v_{1}$ and $v_{2}$ in $V$,

$$
d\left(x_{1}, x_{2}\right)=\frac{\left\|v_{1} \wedge v_{2}\right\|}{\left\|v_{1}\right\|\left\|v_{2}\right\|} .
$$

For any $v \neq 0$ in $V$ and any $\varphi \neq 0$ in $V^{*}$, the quotient

$$
\delta\left(\mathbb{Q}_{p} v, \mathbb{Q}_{p} \varphi\right):=\frac{|\varphi(v)|}{\|\varphi\|\|v\|}
$$

is equal to the distance in $\mathbb{P}(V)$ between $\mathbb{Q}_{p} v$ and $\operatorname{ker} \varphi$ :

$$
\delta\left(\mathbb{Q}_{p} v, \mathbb{Q}_{p} \varphi\right)=d\left(\mathbb{Q}_{p} v, \operatorname{ker} \varphi\right) .
$$

Note that this equals 1 if and only if $\varphi(v) \neq 0$ and the decomposition $V=\mathbb{Q}_{p} v \oplus$ $\operatorname{ker} \varphi$ is good.

Now let $V$ be an irreducible representation of $H$, which therefore factors through $H_{p}$ for some $p$ in $\mathcal{S}$. We endow $V$ with a good norm $\|$.$\| . We may assume that$ this norm is $(K, A)$-good, i.e. that it is $K$-invariant, that, on each weight space for the $A$-action, $Z$ acts by similarities and that the decomposition of $V$ as the sum of these weight spaces is good (see [23]). There exists a highest weight $\chi$ among the 
weights of $A$ in $V$ in the sense that, for any other weight $\chi^{\prime}$ of $A$ in $V, \chi-\chi^{\prime}$ is a linear combination with non-negative integer entries of elements of $\Pi$. Let $V_{\chi}$ be the corresponding weight space of $A$ in $V$ so that $P V_{\chi} \subset V_{\chi}$.

For $\Theta \subset \Pi$, we let $\chi_{\Theta}$ be the restriction of $\chi$ to $A_{\Theta}$ and $V_{\chi_{\Theta}}$ be the corresponding weight space for $A_{\Theta}$ in $V$. Then $V_{\chi \Theta}$ is the sum of weight spaces of $A$ in $V$ which are associated to weights $\chi^{\prime}$ such that $\chi-\chi^{\prime}$ is a linear combination with non-negative integer entries of elements of $\Pi \backslash \Theta$. This space is also exactly the space of fixed points of $U_{\Theta}$ in $V$. For any point $\eta=h \eta_{\Theta}$ of the flag variety $\mathcal{P}_{\Theta}$, we set $V_{\eta}$ to be the vector subspace of $V$ given by $V_{\eta}:=h V_{\chi_{\Theta}}$ : it is invariant under the stabilizer of $\eta$ in $H$. The map $\eta \mapsto V_{\eta}$ is a $H$-equivariant map from $\mathcal{P}_{\Theta}$ to $\operatorname{Gr}(V)$.

In case $\Theta=\Theta_{\mu}$, as usual, we write $\chi_{\mu}=\chi_{\Theta}$, etc. Let us use the objects defined in this section to give estimates for an element of $\Gamma_{\mu}^{+}$of the growth speed in a given representation and of the contraction speed in the associated projective space:

Lemma 4.1 (Contraction and growth in $\mathbb{P}(V)$ ). Let $V$ be an irreducible representation of $H$ with highest weight $\chi$ equipped with a $(K, A)$-good norm. There exists $C>1$ such that, for any $h$ in $\Gamma_{\mu}^{+}$and $v$ in $V \backslash\{0\}$, one has

$$
d\left(\mathbb{Q}_{\mathcal{S}} h v, \mathbb{P}\left(V_{\xi_{h}^{M}}\right)\right) \leq \max _{\alpha \in \Theta_{\mu}} e^{-\alpha^{\omega}(\kappa(h))} d\left(\mathbb{Q}_{\mathcal{S}} v, \mathbb{P}\left(\left(V_{\xi_{h}^{m}}^{*}\right)^{\perp}\right)\right)^{-1}
$$

and

$$
e^{\chi^{\omega}(\kappa(h))} \geq \frac{\|h v\|}{\|v\|} \geq \frac{1}{C} e^{\chi^{\omega}(\kappa(h))} d\left(\mathbb{Q}_{\mathcal{S}} v, \mathbb{P}\left(\left(V_{\xi_{h}^{m}}^{*}\right)^{\perp}\right)\right) .
$$

Proof. Writing $h=k_{h} z_{h} l_{h}$, we are brought back to proving analoguous inequalities when $h$ belongs to $Z^{+}$, the values $\alpha^{\omega}(\kappa(h)), \alpha \in \Pi \backslash \Theta_{\mu}$, are bounded, $V_{\xi_{h}^{M}}$ is replaced by $V_{\chi_{\mu}}$ and $\left(V_{\xi_{h}^{m}}^{*}\right)^{\perp}$ is replaced by the unique $A$-invariant complementary subspace to $V_{\chi_{\mu}}$. In this case, the result follows from direct computations using the definition of the distance in projective space and the fact that the involved decompositions of $V$ are good ones.

For $\beta$-almost every $b \in B$ we set $V_{b}:=V_{\xi_{b}}$ so that $V_{b}=b_{0} V_{T b}$.

Lemma 4.2. Let $V$ be an irreducible representation of $H$ with highest weight $\chi$ equipped with a $(K, A)$-good norm. There exists $C>1$ such that, for $\beta$-almost every $b$ in $B$, for any $n \geq 1$ and $v$ in $V_{T^{n} b}$, one has

$$
e^{\chi^{\omega}\left(\theta_{\mathbb{R}, n}(b)\right)}\|v\| \geq\left\|b_{0} \cdots b_{n-1} v\right\| \geq \frac{1}{C} e^{\chi^{\omega}\left(\theta_{\mathbb{R}, n}(b)\right)}\|v\| .
$$

Proof. By definition, we have

$$
b_{0} \cdots b_{n-1} s\left(\xi_{T^{n} b}\right)=s\left(b_{0} \cdots b_{n-1} \xi_{T^{n} b}\right) \theta_{n}(b) .
$$

Thus, as $s$ takes its values in $K U_{\mu},\left\|b_{0} \cdots b_{n-1} v\right\|=\left\|\theta_{n}(b) s\left(\xi_{T^{n} b}\right)^{-1} v\right\|$. Now, by construction, $s\left(\xi_{T^{n} b}\right)^{-1} v$ belongs to $V_{\chi_{\mu}}$ and $\theta_{n}(b)$ belongs to $L A_{\mu}$, where $L$ is a fixed compact subset of $Z_{\mu}$. The result follows.

Let us now explain how the action of $H$ on $\mathcal{P}_{\mu}$ can be described thanks to the action of $H$ on the projective spaces of finitely many representations. For each simple root $\alpha \in \Pi$, there exists an irreducible representation $\left(\rho_{\alpha}, V_{\alpha}\right)$ whose highest weight $\chi_{\alpha}$ has multiplicity one and is orthogonal to all the other simple roots $\alpha^{\prime} \neq \alpha$. 
When $\alpha$ is in $\Theta_{\mu}$, this representation is $\mu$-proximal, that is, the space $\left(V_{\alpha}\right)_{\left(\chi_{\alpha}\right)_{\mu}}$ also has dimension one. We then have an $H$-equivariant embedding

$$
\mathcal{P}_{\mu} \rightarrow \prod_{\alpha \in \Theta_{\mu}} \mathbb{P}\left(V_{\alpha}\right), \eta \mapsto\left(V_{\alpha, \eta}\right)_{\alpha \in \Theta_{\mu}} .
$$

In the same way, one has an $H$-equivariant embedding

$$
\mathcal{P}_{\breve{\mu}} \rightarrow \prod_{\alpha \in \Theta_{\mu}} \mathbb{P}\left(V_{\alpha}^{*}\right), \eta \mapsto\left(V_{\alpha, \eta}^{*}\right)_{\alpha \in \Theta_{\mu}} .
$$

We endow the representations $V_{\alpha}$ with $(K, A)$-good norms, and we equip $\mathcal{P}_{\mu}$ and $\mathcal{P}_{\check{\mu}}$ with the induced distances. For any $\eta$ in $\mathcal{P}_{\mu}$ and $\zeta$ in $\mathcal{P}_{\check{\mu}}$, we set, using the notation (4.2),

$$
\delta(\eta, \zeta)=\min _{\alpha \in \Theta_{\mu}} \delta\left(V_{\alpha, \eta}, V_{\alpha, \zeta}^{*}\right)
$$

in such a way that one has $\delta(\eta, \zeta) \neq 0$ if and only if $(\eta, \zeta)$ belongs to the open $H$-orbit in $\mathcal{P}_{\mu} \times \mathcal{P}_{\check{\mu}}$. We denote by $\mathcal{Q}_{\zeta} \varsubsetneqq \mathcal{P}_{\mu}$ the maximal Schubert variety given by

$$
\mathcal{Q}_{\zeta}:=\left\{\eta \in \mathcal{P}_{\mu} \mid \delta(\eta, \zeta)=0\right\}
$$

Note that, by construction, for any $h$ in $H$, one has $\mathcal{Q}_{h}^{m}=\mathcal{Q}_{\xi_{h}^{m}}$.

If $\mathcal{Q}$ is a maximal Schubert variety in $\mathcal{P}_{\mu}$ and $\eta$ belongs to $\mathcal{P}_{\mu}$, we shall sometimes use the convention $\delta(\eta, \mathcal{Q})=\delta(\eta, \zeta)$, where $\zeta$ is the unique element of $\mathcal{P}_{\check{\mu}}$ with $\mathcal{Q}=\mathcal{Q}_{\zeta}$

We fix a norm on the real vector space $\mathfrak{a}$. We have the classical

Lemma 4.3 (Contraction and growth in $\mathcal{P}_{\mu}$ ). There exists $C>0$ such that, for all $h$ in $\Gamma_{\mu}^{+}$and $\eta$ in $\mathcal{P}_{\mu}$, one has

$$
d\left(h \eta, \xi_{h}^{M}\right) \leq \max _{\alpha \in \Theta_{\mu}} e^{-\alpha^{\omega}(\kappa(h))} \delta\left(\eta, \mathcal{Q}_{h}^{m}\right)^{-1}
$$

and

$$
\left\|\kappa(h)-\sigma_{\mathbb{R}}(h, \eta)\right\| \leq C\left|\log \left(\delta\left(\eta, \mathcal{Q}_{h}^{m}\right)\right)\right| .
$$

Proof. The first inequality is a translation of Lemma 4.1, applied simultaneously in the representations $V_{\alpha}, \alpha \in \Theta_{\mu}$. The second is proved in the same way, once one has recalled that $\sup _{h \in \Gamma_{\mu}^{+}} d\left(\kappa(h), \mathfrak{a}_{\mu}\right)<\infty$ and that the linear forms $\chi_{\alpha}^{\omega}, \alpha \in \Theta_{\mu}$, span the dual space of $\mathfrak{a}_{\mu}$.

\subsection{Limit laws.}

We now state various limit laws for products of independent identically distributed elements of $H$.

We assume first that $\mu$ admits a finite first moment $\int_{H}\|\kappa(h)\| \mathrm{d} \mu(h)<\infty$. According to Osseledets' theorem, there exists an element $\sigma_{\mu}$ in $\mathfrak{a}_{\mu}$ such that, for $\beta$-almost every $b$ in $B$, one has

$$
\frac{1}{n} \kappa\left(b_{n-1} \cdots b_{0}\right) \underset{n \rightarrow \infty}{\longrightarrow} \sigma_{\mu} .
$$

We have $\sigma_{\mu}=\int_{H \times \mathcal{P}_{\mu}} \sigma_{\mathbb{R}} \mathrm{d}\left(\mu \otimes \nu_{\mu}\right)=\int_{B} \theta_{\mathbb{R}} \mathrm{d} \beta$.

The following statement, originally due to Furstenberg, has been extended to the general case by Guivarc'h and Raugi (see [18, or 9]):

Proposition 4.4 (Simplicity of the Lyapounov exponents). For any $\alpha$ in $\Theta_{\mu}$, one has $\alpha^{\omega}\left(\sigma_{\mu}\right)>0$. 
From now on, we assume that $\mu$ admits a finite exponential moment:

$$
\int_{H} e^{\tau\|\kappa(h)\|} \mathrm{d} \mu(h)<\infty, \text { for some } \tau>0 .
$$

Let $\mathfrak{b}_{\mu}$ be the vector subspace of $\mathfrak{a}_{\mu}$ spanned by elements of the form $\sigma_{\mathbb{R}}\left(\gamma, \xi_{\gamma}^{+}\right)-$ $n \sigma_{\mu}$, where $\gamma$ is an element of $\Gamma_{\mu}^{+}$which can be written as the product of $n$ elements of the support of $\mu$. We set $e_{\mu}:=\operatorname{dim}_{\mathbb{R}} \mathfrak{b}_{\mu}$.

Proposition 4.5 (Central limit theorem). There exists a non-degenerate positive quadratic form $\Phi_{\mu}$ on $\mathfrak{b}_{\mu}$ such that, denoting by $\mathrm{d} T$ the Haar measure on $\mathfrak{b}_{\mu}$ which gives mass 1 to the unit cube for $\Phi_{\mu}$, for $\beta$-almost every $b$ and for all $\varphi \in C_{c}\left(\mathfrak{a}_{\mu}\right)$, one has

$$
\lim _{n \rightarrow \infty} \int_{H} \varphi\left(\frac{1}{\sqrt{n}}\left(\kappa(h)-n \sigma_{\mu}\right)\right) \mathrm{d} \mu^{* n}(h)=\frac{1}{(2 \pi)^{\frac{e_{\mu}}{2}}} \int_{\mathfrak{b}_{\mu}} \varphi(T) e^{-\frac{1}{2} \Phi_{\mu}(T)} \mathrm{d} T .
$$

This proposition is proven in 9 .

The following proposition is the version of the law of the iterated logarithm we will require.

Proposition 4.6 (Law of the iterated logarithm for $\kappa$ ). For $\beta$-almost every $b$ in $B$, one has

$$
\limsup _{n \rightarrow \infty} \Phi_{\mu}\left(\frac{\kappa\left(b_{n-1} \cdots b_{0}\right)-n \sigma_{\mu}}{\sqrt{2 n \log \log n}}\right)=1 .
$$

This proposition is proven in 9 .

We now want to state a local limit theorem.

To deal with the $p$-adic factors of $H$, we need to introduce a group $\Delta_{\mu}$, a vector $v_{\mu}$, a function $\psi$ and a measure $\Pi_{\mu}$. If $\mathcal{S}=\{\infty\}$, we will have $\Delta_{\mu}=\mathfrak{a}_{\mu}, v_{\mu}=0$, $\psi=0$ and $\Pi_{\mu}=\mathrm{d} T$. In general, according to [9], there exists a smallest closed subgroup $\Delta_{\mu}$ of $\mathfrak{b}_{\mu}$ such that there exists $v_{\mu}$ in $\mathfrak{b}_{\mu}$ and a Hölder continuous function $\psi: \mathcal{P}_{\mu} \rightarrow \mathfrak{a}_{\mu}$ such that, for $\mu \otimes \nu_{\mu}$-almost every $(h, \eta)$ in $H \times \mathcal{P}_{\mu}$,

$$
\sigma_{\mathbb{R}}(h, \eta)+\psi(h \eta)-\psi(\eta) \in \sigma_{\mu}+v_{\mu}+\Delta_{\mu} .
$$

The group $\Delta_{\mu}$ is cocompact in $\mathfrak{b}_{\mu}$. We let $\pi_{\mu}$ be the Haar measure of $\Delta_{\mu}$ which gives mass one to the unit cubes of $\Phi_{\mu}$ in the connected component of $\Delta_{\mu}$. Let $\Pi_{\mu}$ be the measure on $\mathfrak{a}_{\mu}$, average of translates of $\pi_{\mu}$, given, for $C \subset \mathfrak{a}_{\mu}$, by

$$
\Pi_{\mu}(C)=\int_{X} \pi_{\mu}(\psi(\eta)+C) \mathrm{d} \nu_{\mu}(\eta) .
$$

For $n \geq 1$ and $\eta \in \operatorname{Supp}\left(\nu_{\mu}\right)$, we introduce the positive measure $\lambda_{n, \eta}$ on $\mathfrak{a}_{\mu}$ given by, for every $\varphi \in C_{c}\left(\mathfrak{a}_{\mu}\right)$,

$$
\lambda_{n, \eta}(\varphi)=\int_{H} \varphi\left(\sigma_{\mathbb{R}}(h, \eta)-n \sigma_{\mu}\right) \mathrm{d} \mu^{* n}(h) .
$$

Here is the version of the local limit theorem with moderate deviations that we will need. We keep the notation of Proposition 4.5

Proposition 4.7 (Local limit theorem). We fix a bounded convex subset $C \subset \mathfrak{a}_{\mu}$, and $\varepsilon>0$. Then one has the limit

$$
\lim _{n \rightarrow \infty}(2 \pi n)^{\frac{e_{\mu}}{2}} e^{\frac{\Phi_{\mu}\left(v_{n}\right)}{2 n}} \lambda_{n, \eta}\left(v_{n}+C\right)-\Pi_{\mu}\left(v_{n}-n v_{\mu}-\psi(\eta)+C\right)=0 .
$$

This limit is uniform for $\eta \in \operatorname{Supp}\left(\nu_{\mu}\right)$ and $v_{n} \in \mathfrak{a}_{\mu}$ with $\left\|v_{n}\right\| \leq \varepsilon \sqrt{n \log n}$.

This proposition is proven in 9 . 


\subsection{Large deviations, spectral gap and Hölder regularity.}

We pursue our description of the asymptotic behavior for products of independent identically distributed elements of $H$. We recall the large deviation principle for the norm of this product, the exponential speed of equidistribution on the flag variety and the Hölder regularity for the Furstenberg stationary measure.

We then use these facts to prove the version of the law of iterated logarithm that we will use in the proof of the "law of the angles" in section 4.7

Proposition 4.8 (Large deviations principle). For every $\varepsilon>0$, there exists $\alpha>0$ and $n_{0}>0$ such that, for all $n \geq n_{0}$ and all $\eta \in \mathcal{P}_{\mu}$, one has

$$
\begin{array}{r}
\mu^{* n}\left(\left\{h \in H \mid\left\|\sigma_{\mathbb{R}}(h, \eta)-n \sigma_{\mu}\right\| \geq n \varepsilon\right\}\right) \leq e^{-n \alpha}, \\
\mu^{* n}\left(\left\{h \in H \mid\left\|\kappa(h)-n \sigma_{\mu}\right\| \geq n \varepsilon\right\}\right) \leq e^{-n \alpha} .
\end{array}
$$

This proposition is proven in 9 .

For $\alpha \in(0,1)$, let us denote by $\mathcal{H}^{\alpha}\left(\mathcal{P}_{\mu}\right)$ the Banach space of $\alpha$-Hölder continuous functions on $\mathcal{P}_{\mu}$ endowed with the norm, given for all $\varphi \in \mathcal{H}^{\alpha}\left(\mathcal{P}_{\mu}\right)$, by

$$
\|\varphi\|_{\alpha}=\sup _{\eta}|\varphi(\eta)|+\sup _{\eta \neq \zeta} \frac{|\varphi(\eta)-\varphi(\zeta)|}{d(\eta, \zeta)^{\alpha}} .
$$

Proposition 4.9 (Exponential speed of equidistribution). For any small enough $\alpha_{1}>0$, there exists $\alpha_{2}>0$ and $n_{0} \geq 1$ such that, for all $\varphi \in \mathcal{H}^{\alpha_{1}}\left(\mathcal{P}_{\mu}\right)$, all $n \geq n_{0}$, and all $\eta \in \mathcal{P}_{\mu}$, one has

$$
\left|\int_{H} \varphi(h \eta) \mathrm{d} \mu^{* n}(h)-\int_{\mathcal{P}_{\mu}} \varphi(\zeta) \mathrm{d} \nu_{\mu}(\zeta)\right| \leq e^{-n \alpha_{2}}\|\varphi\|_{\alpha_{1}} .
$$

This is a direct consequence of the spectal gap for the Markov operator on the space $\mathcal{H}^{\alpha}\left(H / P_{\mu}\right)$ (see [9]).

Proposition 4.10 (Hölder regularity of $\nu_{\mu}$ ). For $\alpha_{3}>0$ sufficiently small and for all $\zeta \in \mathcal{P}_{\breve{\mu}}$, one has $\nu_{\mu}\left(\mathcal{Q}_{\zeta}\right)=0$ and

$$
\int_{\mathcal{P}_{\mu}} \delta(\eta, \zeta)^{-\alpha_{3}} \mathrm{~d} \nu_{\mu}(\eta)<\infty
$$

This proposition is proven in 9 .

Corollary 4.11. For $\alpha_{3}>0$ sufficiently small, there exists a constant $C_{3} \geq 1$ such that one has, for all $\varepsilon>0$ and all maximal Schubert varieties $\mathcal{Q} \varsubsetneqq \mathcal{P}_{\mu}$,

$$
\nu_{\mu}\left(\left\{\zeta \in \mathcal{P}_{\mu} \mid \delta(\zeta, \mathcal{Q}) \leq \varepsilon\right\}\right) \leq C_{3} \varepsilon^{\alpha_{3}} .
$$

This corollary is a straightforward application of Proposition 4.10, Note that this corollary is more precise than Corollary 4.2 of [11] since here we give an upper bound for the mass of a neighborhood of a maximal Schubert variety instead of the neighborhood of a point.

Recall that the function $\theta$ on $B$ has been defined in (4.1).

Corollary 4.12 (Law of the iterated logarithm for $\theta_{\mathbb{R}}$ ). For $\beta$-almost every $b$ in $B$, one has

$$
\limsup _{n \rightarrow \infty} \Phi_{\mu}\left(\frac{\theta_{\mathbb{R}, n}(b)-n \sigma_{\mu}}{\sqrt{2 n \log \log n}}\right)=1
$$


The proof relies on the following.

Lemma 4.13. For $\beta$-almost every b, one has

$$
\limsup _{n \rightarrow \infty} \frac{\left|\log \delta\left(\xi_{T^{n} b}, \mathcal{Q}_{b_{0} \cdots b_{n-1}}^{m}\right)\right|}{\log n}<\infty .
$$

Proof. Fix $C>1$. For any integer $n$, we have

$$
\begin{aligned}
\beta\left(\left\{b \in B \mid \delta\left(\xi_{T^{n} b}, \mathcal{Q}_{b_{0} \cdots b_{n-1}}^{m}\right) \leq\right.\right. & \left.\left.n^{-C}\right\}\right) \\
& =\int_{H} \beta\left(\left\{b \in B \mid \delta\left(\xi_{T^{n} b}, \mathcal{Q}_{h}^{m}\right) \leq n^{-C}\right\}\right) \mathrm{d} \mu^{* n}(h) .
\end{aligned}
$$

As $\xi_{*} T_{*}^{n} \beta=\xi_{*} \beta=\nu_{\mu}$, by Corollary 4.11, there exists $\alpha>0$ and $n_{0} \geq 1$ such that, for all $n \geq n_{0}$ and $h \in H$, one has

$$
\begin{aligned}
\beta\left(\left\{b \in B \mid \delta\left(\xi_{T^{n} b}, \mathcal{Q}_{h}^{m}\right) \leq n^{-C}\right\}\right) & =\nu_{\mu}\left(\left\{\zeta \in \mathcal{P}_{\mu} \mid \delta\left(\zeta, \mathcal{Q}_{h}^{m}\right) \leq n^{-C}\right\}\right) \\
& \leq n^{-C \alpha}
\end{aligned}
$$

One chooses $C>\alpha^{-1}$ so that the series $\sum_{n} n^{-C \alpha}$ converges. Then, by the BorelCantelli Lemma, for $\beta$-almost every $b$ in $B$, one has, for $n$ large,

$$
\delta\left(\xi_{T^{n} b}, \mathcal{Q}_{b_{0} \cdots b_{n-1}}^{m}\right) \geq n^{-C} .
$$

Proof of Corollary 4.12. Recall that $\iota: \mathfrak{a} \rightarrow \mathfrak{a}$ denotes the opposition involution. One easily checks that one has $\sigma_{\breve{\mu}}=\iota\left(\sigma_{\mu}\right)$ and $\Phi_{\breve{\mu}}=\Phi_{\mu} \circ \iota$. Thus, applying Proposition 4.6 to $\check{\mu}$, one gets, as for any $h$ in $H, \kappa\left(h^{-1}\right)=\iota(\kappa(h))$, for $\beta$-almost any $b$ in $B$,

$$
\limsup _{n \rightarrow \infty} \Phi_{\mu}\left(\frac{\kappa\left(b_{0} \cdots b_{n-1}\right)-n \sigma_{\mu}}{\sqrt{2 n \log \log n}}\right)=1 .
$$

But, from Lemmas 4.3 and 4.13 , we get

$$
\limsup _{n \rightarrow \infty} \Phi_{\mu}\left(\frac{\kappa\left(b_{0} \cdots b_{n-1}\right)-\theta_{\mathbb{R}, n}(b)}{\log n}\right)<\infty .
$$

The conclusion follows.

\subsection{Exponential convergence of the density points $\xi_{h}^{M}$.}

We use the results of the previous section to obtain a control with an exponential precision on the position of the density point $\xi_{h}^{M}$ of a word $h$ of length $n$ outside a set whose $\beta$-mass decreases exponentially with $n$ (Corollary 4.18). Similar results when $H$ is a real semisimple group were obtained independently by Richard Aoun in 1 .

We will use this corollary to prove the "law of the angle" in section 4.7 .

Lemma 4.14. There exist constants $\alpha_{1}, \alpha_{2}, \alpha_{3} \in(0,1), C_{0}>0$ and $n_{0} \geq 1$ such that, for all $\left.n \geq n_{0}, \varepsilon \in\right] 0,1\left[, \eta \in \mathcal{P}_{\mu}\right.$ and $\mathcal{Q} \varsubsetneqq \mathcal{P}_{\mu}$ a maximal Schubert variety, one has

$$
\mu^{* n}(\{h \in H \mid \delta(h \eta, \mathcal{Q}) \leq \varepsilon\}) \leq C_{0}\left(\varepsilon^{\alpha_{3}}+e^{-n \alpha_{2}} \varepsilon^{-\alpha_{1}}\right) .
$$


Proof. Let $\rho:[0, \infty) \rightarrow[0,1]$ be the piecewise affine function such that $\rho(t)=1$ for $t \in[0,1], \rho(t)=2-t$ for $t \in[1,2]$ and $\rho(t)=0$ for $t \geq 2$. We choose positive constants $\alpha_{1}, \alpha_{2}$ from Proposition 4.9 and positive constants $\alpha_{3}, C_{3}$ from Corollary 4.11. There exists a constant $C_{0} \geq 2 C_{3}$ such that for any $\left.\varepsilon \in\right] 0,1$ [, for any maximal Schubert variety $\mathcal{Q} \varsubsetneqq \mathcal{P}_{\mu}$, the function $\varphi: \mathcal{P}_{\mu} \rightarrow[0,1] ; \eta \mapsto \varphi(\eta):=\rho\left(\frac{1}{\varepsilon} \delta(\eta, \mathcal{Q})\right)$ is $\alpha_{1}$-Hölder continuous and satisfies $\|\varphi\|_{\alpha_{1}} \leq C_{0} \varepsilon^{-\alpha_{1}}$. Then for $n$ large enough, for all $\eta \in \mathcal{P}_{\mu}$, one has

$$
\begin{aligned}
\mu^{* n}(\{h \in H \mid \delta(h \eta, \mathcal{Q}) \leq \varepsilon\}) & \leq \int_{H} \varphi(h \eta) \mathrm{d} \mu^{* n}(h) \\
& \leq \int_{\mathcal{P}_{\mu}} \varphi(\zeta) \mathrm{d} \nu_{\mu}(\zeta)+e^{-n \alpha_{2}}\|\varphi\|_{\alpha_{1}} \\
& \leq C_{0}\left(\varepsilon^{\alpha_{3}}+e^{-n \alpha_{2}} \varepsilon^{-\alpha_{1}}\right) .
\end{aligned}
$$

This proves our claim.

Corollary 4.15. For every $\alpha_{4}>0$ there exists a constant $\alpha_{5}>0$ and an integer $n_{0} \geq 1$ such that, for $n \geq n_{0}$, for all $\eta \in \mathcal{P}_{\mu}$ and all maximal Schubert variety $\mathcal{Q} \varsubsetneqq \mathcal{P}_{\mu}$, one has

$$
\begin{gathered}
\mu^{* n}\left(\left\{h \in H \mid \delta(h \eta, \mathcal{Q}) \leq e^{-n \alpha_{4}}\right\}\right) \leq e^{-n \alpha_{5}}, \\
\mu^{* n}\left(\left\{h \in H \mid \delta\left(\xi_{h}^{M}, \mathcal{Q}\right) \leq e^{-n \alpha_{4}}\right\}\right) \leq e^{-n \alpha_{5}}, \\
\mu^{* n}\left(\left\{h \in H \mid \delta\left(\eta, \mathcal{Q}_{h}^{m}\right) \leq e^{-n \alpha_{4}}\right\}\right) \leq e^{-n \alpha_{5}} .
\end{gathered}
$$

Proof. ( $i$ ) This inequality is a consequence of Lemma 4.14 applied with $\varepsilon=e^{-n \alpha_{4}}$. We may assume $\alpha_{4}$ small enough to have $\alpha_{1} \alpha_{4}<\alpha_{2}$. We then choose $\alpha_{5}=$ $\frac{1}{2} \min \left(\alpha_{3} \alpha_{4}, \alpha_{2}-\alpha_{1} \alpha_{4}\right)$.

(ii) This is a consequence of $(i)$ and of the large deviations principle. We note that the set of maximal Schubert subvarieties $\mathcal{Q} \varsubsetneqq \mathcal{P}_{\mu}$ is compact for the Hausdorff topology. Hence there exists $\varepsilon_{0}>0$ and a finite set $F$ of points $\eta$ in $\mathcal{P}_{\mu}$ such that for any maximal Schubert variety $\mathcal{Q} \varsubsetneqq \mathcal{P}_{\mu}$, some $\eta$ in $F$ satisfies $d(\eta, \mathcal{Q}) \geq \varepsilon_{0}$. Thus, by Lemma 4.3, for any $h \in H$ and for at least one of these points $\eta \in F$, one has $\delta\left(\eta, Q_{h}^{m}\right) \geq \varepsilon_{0}$, and hence,

$$
\delta\left(\xi_{h}^{M}, h \eta\right) \leq \varepsilon_{0}^{-1} \max _{\alpha \in \Theta_{\mu}} e^{-\alpha^{\omega}(\kappa(h))} .
$$

Applying Propositions 4.4 and 4.8, one finds constants $t_{1}>0, \alpha_{5}>0$ and $n_{0}>0$ such that, for $n \geq n_{0}$, one has

$$
\mu^{* n}\left(\left\{h \in H \mid \forall \eta \in F \quad d\left(\xi_{h}^{M}, h \eta\right) \geq e^{-n t_{1}}\right\}\right) \leq e^{-n \alpha_{5}} / 2 .
$$

By Corollary 4.15(i), after eventually taking a smaller $\alpha_{5}>0$ and a larger $n_{0}>0$, one can also assume that, for $n \geq n_{0}$, one has

$$
\mu^{* n}\left(\left\{h \in H \mid \exists \eta \in F \quad \delta(h \eta, \mathcal{Q}) \leq 2 e^{-n \alpha_{4}}\right\}\right) \leq e^{-n \alpha_{5}} / 2 .
$$

Our claim is a consequence of the two inequalities (4.6) and (4.6), since we can choose $\alpha_{4}$ such that $\alpha_{4}<t_{1}$.

(iii) This statement follows from (ii) applied to $\check{\mu}$.

Corollary 4.16. There exists a constant $\alpha_{6}>0$ and an integer $n_{0} \geq 1$ such that, for $n \geq n_{0}$ and for all $\eta \in \mathcal{P}_{\mu}$,

$$
\mu^{* n}\left(\left\{h \in H \mid d\left(h \eta, \xi_{h}^{M}\right) \geq e^{-n \alpha_{6}}\right\}\right) \leq e^{-n \alpha_{6}} .
$$


Proof. Again by Lemma 4.3, for all $h$ in $H$ and $\eta$ in $\mathcal{P}_{\mu}$, one has

$$
d\left(h \eta, \xi_{h}^{M}\right) \leq \max _{\alpha \in \Theta_{\mu}} e^{-\alpha^{\omega}(\kappa(h))} \delta\left(\eta, \mathcal{Q}_{h}^{m}\right)^{-1} .
$$

Again by Propositions 4.4 and 4.8, there exist constants $t_{1}>0$ and $\alpha>0$ and subsets $E_{n}$ of $H$ with $\mu^{* n}\left(E_{n}^{c}\right)=O\left(e^{-\alpha n}\right)$ such that for all $h \in E_{n}$ one has $\min _{\alpha \in \Theta_{\mu}} \alpha^{\omega}(\kappa(h)) \geq t_{1} n$.

Applying Corollary 4.15 (iii) with $\alpha_{4}<t_{1}$, one gets a constant $\alpha_{5}>0$ and a subset $F_{n}$ of $H$ with $\mu^{* n}\left(F_{n}^{c}\right)=O\left(e^{-\alpha_{5} n}\right)$ such that for all $h \in F_{n}$ one has $\delta\left(\eta, \mathcal{Q}_{h}^{m}\right) \geq e^{-n \alpha_{4}}$. Hence, using Lemma 4.3. for $h$ in both $E_{n}$ and $F_{n}$, one has $d\left(h \eta, \xi_{h}^{M}\right) \leq e^{-\left(t_{1}-\alpha_{4}\right) n}$. One chooses $\alpha_{6}=\frac{1}{2} \min \left(\alpha, \alpha_{5}, t_{1}-\alpha_{4}\right)$.

The next proposition is the main result of this section. It gives a quantitative version of the statement that the density point $\xi_{h_{1} \cdots h_{p}}^{M}$ of a random word $h_{1} \cdots h_{p}$ mainly depends on the first letters $h_{1}, \ldots, h_{n}$.

Proposition 4.17. There exist a constant $\alpha_{7}>0$ and an integer $n_{0} \geq 1$ such that, for all $p \geq n \geq n_{0}$,

$$
\mu^{\otimes p}\left(\left\{\left(h_{1}, \ldots, h_{p}\right) \in H^{p} \mid d\left(\xi_{h_{1} \cdots h_{p}}^{M}, \xi_{h_{1} \cdots h_{n}}^{M}\right) \geq e^{-n \alpha_{7}}\right\}\right) \leq e^{-n \alpha_{7}} .
$$

Proof. Fix a point $\eta \in \mathcal{P}_{\mu}$. According to Corollary 4.16 applied to the points $h_{n+1} \cdots h_{p} \eta \in \mathcal{P}_{\mu}$, there exist $\alpha_{6}>0$ and $n_{0} \geq 1$ such that, for $n_{0} \leq n \leq p$ and all $\left(h_{n+1}, \ldots, h_{p}\right) \in H^{p-n}$,

$$
\mu^{\otimes n}\left(\left\{\left(h_{1}, \ldots, h_{n}\right) \in H^{n} \mid d\left(h_{1} \cdots h_{p} \eta, \xi_{h_{1} \cdots h_{n}}^{M}\right) \geq e^{-n \alpha_{6}}\right\}\right) \leq e^{-n \alpha_{6}} .
$$

Averaging with respect to the law $\mu^{\otimes(p-n)}$, one gets

$$
\mu^{\otimes p}\left(\left\{\left(h_{1}, \ldots, h_{p}\right) \in H^{p} \mid d\left(h_{1} \cdots h_{p} \eta, \xi_{h_{1} \cdots h_{n}}^{M}\right) \geq e^{-n \alpha_{6}}\right\}\right) \leq e^{-n \alpha_{6}} .
$$

In the special case where $p=n$, this can be written as

$$
\mu^{\otimes p}\left(\left\{\left(h_{1}, \ldots, h_{p}\right) \in H^{p} \mid d\left(h_{1} \cdots h_{p} \eta, \xi_{h_{1} \cdots h_{p}}^{M}\right) \geq e^{-p \alpha_{6}}\right\}\right) \leq e^{-p \alpha_{6}} .
$$

Combining these last two inequalities, one gets

$$
\mu^{\otimes p}\left(\left\{\left(h_{1}, \ldots, h_{p}\right) \in H^{p} \mid d\left(\xi_{h_{1} \cdots h_{p}}^{M}, \xi_{h_{1} \cdots h_{n}}^{M}\right) \geq 2 e^{-n \alpha_{6}}\right\}\right) \leq 2 e^{-n \alpha_{6}} .
$$

To conclude, one just has to take $\alpha_{7}=\frac{1}{2} \alpha_{6}$ and $n$ large enough.

Corollary 4.18 (Convergence of the density points). There exist a constant $\alpha_{8}>0$ and an integer $n_{0} \geq 1$ such that, for all $n \geq n_{0}$,

$$
\beta\left(\left\{b \in B \mid \forall p \geq n, d\left(\xi_{b_{0} \cdots b_{p-1}}^{M}, \xi_{b}\right) \leq e^{-p \alpha_{8}}\right\}\right) \geq 1-e^{-n \alpha_{8}} .
$$

Proof. Using the equality $\xi_{b}=b_{0} \cdots b_{n-1} \xi_{T^{n} b}$ for $\beta$-almost every $b \in B$ and applying Corollary 4.16 with the point $\eta=\xi_{T^{n} b}$, one gets the existence of a constant $\alpha_{6}>0$ such that, for $n$ large,

$$
\beta\left(\left\{b \in B \mid d\left(\xi_{b_{0} \cdots b_{n-1}}^{M}, \xi_{b}\right) \geq e^{-n \alpha_{6}}\right\}\right) \leq e^{-n \alpha_{6}} .
$$

Hence

$$
\beta\left(\left\{b \in B \mid \exists p \geq n, d\left(\xi_{b_{0} \cdots b_{p-1}}^{M}, \xi_{b}\right) \geq e^{-p \alpha_{6}}\right\}\right) \leq \sum_{p \geq n} e^{-p \alpha_{6}}=\frac{e^{-n \alpha_{6}}}{1-e^{-\alpha_{6}}} .
$$

One concludes by again taking $\alpha_{8}=\frac{1}{2} \alpha_{6}$ and $n$ large enough. 


\subsection{The law of the angles.}

The aim of this section is the law of the angles. This law is the asymptotic law with respect to the conditional probability measures $\beta_{n, b}^{U}$ as $n$ goes to $\infty$ of the density points $\xi_{b_{0}^{\prime} \cdots b_{n-1}^{\prime}}^{m}$. It plays a crucial role in the drift argument of section 8.1 .

Let us consider the dynamical system $\left(B^{\theta}, \mathcal{B}^{\theta}, \beta^{\theta}, T^{\theta}\right)$ introduced in section 3.3 where $Z=Z_{\mu}$ and $\theta: B \rightarrow Z_{\mu}$ is the function defined in equation (4.1). The space $B^{\theta}$ is the product $B \times Z_{\mu}$, the $\sigma$-algebra $\mathcal{B}^{\theta}$ is the product $\sigma$-algebra, the measure $\beta^{\theta}$ is the product $\beta \otimes \lambda_{Z_{\mu}}$ and the transformation $T^{\theta}$ is given, for $(b, z) \in B^{\theta}$, by $T^{\theta}(b, z)=\left(T b, \theta(b)^{-1} z\right)$.

Let $\mathcal{Q}_{n}^{\theta}$ be the $\sigma$-algebra $\mathcal{Q}_{n}^{\theta}:=\left(T^{\theta}\right)^{-n}\left(\mathcal{B}^{\theta}\right)$, let $C$ be a ball of radius $R$ in $\mathfrak{a}_{\mu}$ and set $U$ to be the open neighborhood of the identity in $Z_{\mu}$ given by $U:=\omega_{\mu}^{-1}(C)$. We choose $R$ so that every open ball in $\mathfrak{b}_{\mu}$ of radius $R-2\|\psi\|_{\infty}$ meets $\Delta_{\mu}$. This condition will ensure, at the very end of this section, that the denominator of the ratio $F_{n, 2}(a)$ is uniformly bounded below. Let $\mathcal{Q}_{n}^{U}$ be the $\sigma$-algebra which is the restriction of $\mathcal{Q}_{n}^{\theta}$ to $B^{U}:=B \times U$ and, for $\beta^{U}$-almost every $c=(b, z)$ in $B \times U$, let $\beta_{n, c}^{U}$ be the conditional probability measure of $\beta^{U}$ at $c$ along $\mathcal{Q}_{n}^{U}$.

The following theorem identifies the asymptotic law of the density points $\xi_{b_{n-1}^{\prime}}^{M} \cdots b_{0}^{\prime}$ as the $\mu$-stationary probability measure $\nu_{\mu}$ and the law of the density points for the inverses $\xi_{b_{0}^{\prime} \cdots b_{n-1}^{\prime}}^{m}=\xi_{\left(b_{n-1}^{\prime}\right)^{-1} \ldots\left(b_{0}^{\prime}\right)^{-1}}^{M}$ as the $\check{\mu}$-stationary probability measure $\nu_{\check{\mu}}$.

Theorem 4.19 (Law of the angles). For $\beta^{U}$-almost every $c=(b, z)$ in $B^{U}$, one has, for any continuous function $\varphi$ on $\mathcal{P}_{\mu}$,

$$
\int_{B^{U}} \varphi\left(\xi_{b_{n-1}^{\prime}}^{M} \ldots b_{0}^{\prime}\right) \mathrm{d} \beta_{n, c}^{U}\left(b^{\prime}, z^{\prime}\right) \underset{n \rightarrow \infty}{\longrightarrow} \int_{\mathcal{P}_{\mu}} \varphi \mathrm{d} \nu_{\mu}
$$

and, for any continuous function $\varphi$ on $\mathcal{P}_{\check{\mu}}$,

$$
\int_{B^{U}} \varphi\left(\xi_{b_{0}^{\prime} \cdots b_{n-1}^{\prime}}^{m}\right) \mathrm{d} \beta_{n, c}^{U}\left(b^{\prime}, z^{\prime}\right) \underset{n \rightarrow \infty}{\longrightarrow} \int_{\mathcal{P}_{\check{\mu}}} \varphi \mathrm{d} \nu_{\check{\mu}}
$$

Proof of Theorem 4.19. We will only prove (4.7) since the proof of (4.8) is similar. Set

$$
q_{n}:=[\log n]^{2} \text { and } p_{n}:=n-q_{n} .
$$

Equation (4.7) is a consequence of the following two equations:

$$
\begin{gathered}
\int_{B^{U}}\left|\varphi\left(\xi_{b_{n-1}^{\prime} \cdots b_{0}^{\prime}}^{M}\right)-\varphi\left(\xi_{b_{n-1}^{\prime} \cdots b_{p_{n}}^{\prime}}^{M}\right)\right| \mathrm{d} \beta_{n, c}^{U}\left(b^{\prime}, z^{\prime}\right) \underset{n \rightarrow \infty}{\longrightarrow} 0, \\
\int_{U} \varphi\left(\xi_{b_{n-1}^{\prime} \cdots b_{p_{n}}^{\prime}}^{M}\right) \mathrm{d} \beta_{n, c}^{U}\left(b^{\prime}, z^{\prime}\right) \underset{n \rightarrow \infty}{\longrightarrow} \nu_{\mu}(\varphi) .
\end{gathered}
$$

To prove (4.10), we only have to check that, for any $\varepsilon>0$,

$$
\beta_{n, c}^{U}\left(\left\{\left(b^{\prime}, z^{\prime}\right) \in B^{U} \mid d\left(\xi_{b_{n-1}^{\prime}}^{M} \cdots b_{0}^{\prime}, \xi_{b_{n-1}^{\prime} \cdots b_{p_{n}}^{\prime}}^{M}\right) \geq \varepsilon\right\}\right) \underset{n \rightarrow \infty}{\longrightarrow} 0 .
$$

According to (3.8), the left-hand side of (4.12) equals

$$
\frac{\mu^{\otimes n}\left(\left\{\left(h_{1}, \ldots, h_{n}\right) \in Q_{n, c}^{U} \mid d\left(\xi_{h_{n} \cdots h_{1}}^{M}, \xi_{h_{n} \cdots h_{p_{n}+1}}^{M}\right) \geq \varepsilon\right\}\right)}{\mu^{\otimes n}\left(Q_{n, c}^{U}\right)},
$$


where, setting $z_{\mathbb{R}}:=\omega_{\mu}(z)$,

$$
Q_{n, c}^{U}=\left\{h \in H^{n} \mid \sigma_{\mathbb{R}}\left(h_{1}, \ldots, h_{n}, \xi_{T^{n} b}\right) \in \theta_{\mathbb{R}, n}(b)-z_{\mathbb{R}}+C\right\} .
$$

On one hand, by Proposition 4.17, the numerator in (4.13) is, for $n$ large, bounded above by $e^{-\alpha_{7} q_{n}}$. Hence by our choice (4.9) of $q_{n}$, it is bounded above by any negative power of $n$. On the other hand, according to the law of the iterated logarithm for $\theta_{\mathbb{R}}$ (Corollary 4.12), for $\beta$-almost every $b \in B$, one has

$$
\left\|\theta_{\mathbb{R}, n}(b)-n \sigma_{\mu}\right\|=O(\sqrt{n \log \log n}) .
$$

Thus, by our choice of $R$ and by the local limit theorem (Proposition 4.7), the denominator in (4.13) is, for $n$ large, bounded below by a negative power of $n$. This proves (4.10).

Let us now deal with (4.11). For $a$ in $B$, set

$$
Q_{n, c, a}^{U}=\left\{h \in H^{p_{n}} \mid \sigma_{\mathbb{R}}\left(h_{1} \cdots h_{p_{n}} a_{q_{n}-1} \cdots a_{0}, \xi_{T^{n} b}\right) \in \theta_{\mathbb{R}, n}(b)-z_{\mathbb{R}}+C\right\} .
$$

Again by (3.7), the left-hand side of (4.11) equals

$$
\int_{B} \varphi\left(\xi_{a_{0} \cdots a_{q_{n}-1}}^{M}\right) \frac{\mu^{\otimes p_{n}}\left(Q_{n, c, a}^{U}\right)}{\mu^{\otimes n}\left(Q_{n, c}^{U}\right)} \mathrm{d} \beta(a) .
$$

As, for $\beta$-almost any $a$ in $B, \xi_{a_{0} \cdots a_{q-1}}^{M} \underset{q \rightarrow \infty}{\longrightarrow} \xi_{a}$ (for example by Corollary 4.18) and $\xi_{*} \beta=\nu_{\mu}$, (4.11) follows from Lemma 4.20 below.

Lemma 4.20. The sequence of functions

$$
F_{n}: a \mapsto \frac{\mu^{\otimes p_{n}}\left(Q_{n, c, a}^{U}\right)}{\mu^{\otimes n}\left(Q_{n, c}^{U}\right)}
$$

goes to 1 in $\mathrm{L}^{1}(B, \mathcal{B}, \beta)$ as $n \rightarrow \infty$.

Proof. For $n>0, a$ in $B$ and $h_{1}, \ldots, h_{p_{n}}$ in $H$, we have

$$
\begin{aligned}
\sigma_{\mathbb{R}}\left(h_{1} \cdots h_{p_{n}} a_{q_{n}-1} \cdots a_{0}, \xi_{T^{n} b}\right)=\sigma_{\mathbb{R}}\left(h_{1} \cdots h_{p_{n}}, a_{q_{n}-1}\right. & \left.\cdots a_{0} \xi_{T^{n} b}\right) \\
& +\sigma_{\mathbb{R}}\left(a_{q_{n}-1} \cdots a_{0}, \xi_{T^{n} b}\right) .
\end{aligned}
$$

Now, by the large deviation principle (Proposition 4.8), there exist subsets $E_{n}$ of $B$ and $\alpha>0$ such that $\beta\left(E_{n}^{c}\right)=O\left(e^{-\alpha q_{n}}\right)$ and that, for all $a$ in $E_{n}$,

$$
\sigma_{\mathbb{R}}\left(a_{q_{n}-1} \cdots a_{0}, \xi_{T^{n} b}\right)-q_{n} \sigma_{\mu}=O\left((\log n)^{2}\right) .
$$

As above, by Corollary 4.12 and Proposition 4.7, one has

$$
\int_{E_{n}^{c}} F_{n} \mathrm{~d} \beta \leq \frac{\beta\left(E_{n}^{c}\right)}{\mu^{\otimes n}\left(Q_{n, c}^{U}\right)} \underset{n \rightarrow \infty}{\longrightarrow} 0 .
$$

Set

$$
v_{n}=\theta_{\mathbb{R}, n}(b)-n \sigma_{\mu}-z_{\mathbb{R}}
$$

and, for $a$ in $E_{n}$,

$$
w_{n}=\sigma_{\mathbb{R}}\left(a_{q_{n}-1} \cdots a_{0}, \xi_{T^{n} b}\right)-q_{n} \sigma_{\mu} .
$$

By Corollary 4.12 and (4.14), uniformly for $a \in E_{n}$, we have

$$
\left\|v_{n}\right\|=O(\sqrt{n \log \log n}) \text { and }\left\|w_{n}\right\|=O\left((\log n)^{2}\right)
$$


so that, by Proposition 4.7 on $E_{n}$ the function $F_{n}$ is uniformly equivalent to the product $F_{n, 1} F_{n, 2}$ where

$$
F_{n, 1}(a)=\frac{e^{-\frac{1}{2 p_{n}} \Phi_{\mu}\left(v_{n}-w_{n}\right)}}{e^{-\frac{1}{2 n} \Phi_{\mu}\left(v_{n}\right)}} \quad, \quad F_{n, 2}(a)=\frac{\Pi_{\mu}\left(v_{n}-w_{n}-p_{n} v_{\mu}-\psi\left(\eta_{n}\right)+C\right)}{\Pi_{\mu}\left(v_{n}-n v_{\mu}-\psi\left(\xi_{T^{n} b}\right)+C\right)}
$$

and $\eta_{n}=a_{q_{n}-1} \cdots a_{0} \xi_{T^{n} b}$. By (4.16), we have

$$
\lim _{n \rightarrow \infty} \frac{q_{n}\left\|v_{n}\right\|^{2}}{n^{2}}=\lim _{n \rightarrow \infty} \frac{\left\|v_{n}\right\|\left\|w_{n}\right\|}{n}=\lim _{n \rightarrow \infty} \frac{\left\|w_{n}\right\|^{2}}{n}=0 ;
$$

hence $F_{n, 1}(a) \underset{n \rightarrow \infty}{\longrightarrow} 1$ uniformly for $a$ in $E_{n}$. When $\mathcal{S}=\{\infty\}$, the measure $\Pi_{\mu}=\pi_{\mu}$ is invariant by all the translations of $\mathfrak{a}_{\mu}$ and $F_{n, 2}=1$.

In general, $\Pi_{\mu}$ is invariant only by the translations of the cocompact subgroup $\Delta_{\mu}$ of $\mathfrak{b}_{\mu}$. However, according to (4.5) and (4.15), the element $w_{n}+\psi\left(\eta_{n}\right)-$ $\psi\left(\xi_{T^{n} b}\right)-q_{n} v_{\mu}$ belongs to $\Delta_{\mu}$. Hence one still has $F_{n, 2}=1$.

\subsection{Conditional behavior of the random walk.}

Using the "law of the angles" (Theorem 4.19), in this section we control the norms and the attracting directions in a given representation of $H$ of most of the words with respect to the conditional probability measures $\beta_{n, c}^{U}$.

Proposition 4.21 below is similar to Corollary 5.5 of 4 . The main difference is that we are no longer dealing with products of independent identically distributed elements of $H$.

Proposition 4.21 (Drift control). Let $V$ be an irreducible representation of $H$. For every $\alpha>0$, there exists $r_{0} \geq 1$ such that, for $\beta^{U}$-almost every $c=(b, z) \in B^{U}$, there exists $n_{0} \geq 1$ such that for $n \geq n_{0}$, for any $v$ in $V \backslash\{0\}$ and any $\eta$ in $\mathcal{P}_{\mu}$, one has, with $c^{\prime}=\left(b^{\prime}, z^{\prime}\right)$,

$$
\beta_{n, c}^{U}\left(\left\{c^{\prime} \in B^{U} \mid\left\|b_{0}^{\prime} \cdots b_{n-1}^{\prime} v\right\| \geq \frac{1}{r_{0}}\left\|b_{0}^{\prime} \cdots b_{n-1}^{\prime}\right\|\|v\|\right\}\right) \geq 1-\alpha
$$

and

$$
\beta_{n, c}^{U}\left(\left\{c^{\prime} \in B^{U} \mid d\left(\mathbb{Q}_{\mathcal{S}} b_{0}^{\prime} \cdots b_{n-1}^{\prime} v, b_{0}^{\prime} \cdots b_{n-1}^{\prime} \mathbb{P}\left(V_{\eta}\right)\right) \leq \alpha\right\}\right) \geq 1-\alpha .
$$

Recall that, in section 4.3 we have defined the map

$$
\mathcal{P}_{\mu} \rightarrow \operatorname{Gr}(\mathrm{V}) ; \eta \mapsto V_{\eta}
$$

as the map sending some $\eta=h \eta_{\Theta_{\mu}}$ to the space $h V^{U_{\mu}}$, where $V^{U_{\mu}}$ is the space of $U_{\mu}$-invariant vectors in $V$.

Proof of Proposition 4.21. We first prove (4.17). For any $x$ in $\mathbb{P}(V)$, set

$$
\mathcal{Q}_{x}:=\left\{\zeta \in \mathcal{P}_{\breve{\mu}} \mid x \subset\left(V_{\zeta}^{*}\right)^{\perp}\right\} .
$$

As $\mathcal{Q}_{x}$ is a proper algebraic subvariety of $\mathcal{P}_{\breve{\mu}}$, we have $\nu_{\breve{\mu}}\left(\mathcal{Q}_{x}\right)=0$. For $\varepsilon>0$, set

$$
\mathcal{Q}_{x}^{\varepsilon}=\left\{\zeta \in \mathcal{P}_{\breve{\mu}} \mid d\left(x, \mathbb{P}\left(\left(V_{\zeta}^{*}\right)^{\perp}\right)\right) \leq \varepsilon\right\} .
$$

According to (4.4) in Lemma 4.1, to prove (4.17) it suffices to establish that, for $\beta^{U}$-almost any $c=(b, z)$ in $B^{U}$,

$$
\lim _{\varepsilon \rightarrow 0} \limsup _{n \rightarrow \infty} \sup _{x \in \mathbb{P}(V)} \beta_{n, c}^{U}\left\{\left(b^{\prime}, z^{\prime}\right) \in B^{U} \mid \xi_{b_{0}^{\prime} \cdots b_{n-1}^{\prime}}^{m} \in \mathcal{Q}_{x}^{\varepsilon}\right\}=0 .
$$


We shall prove this statement by contradiction. By Theorem 4.19, for $\beta^{U}$-almost every $c=(b, z)$ in $B^{U}$, (4.8) holds for any continuous function $\varphi$ on $\mathcal{P}_{\breve{\mu}}$. Assume (4.19) does not hold for such a $c$. Then, there would exist sequences $\left(\varepsilon_{k}\right)$ of positive numbers, $\left(n_{k}\right)$ of integers and points $x_{k} \in \mathbb{P}(V)$ with $\varepsilon_{k} \rightarrow 0$ and $n_{k} \rightarrow \infty$ and

$$
\rho=\inf _{k} \beta_{n_{k}, c}^{U}\left\{\left(b^{\prime}, z^{\prime}\right) \in B^{U} \mid \xi_{b_{0}^{\prime} \ldots b_{n_{k}-1}^{\prime}}^{m} \in \mathcal{Q}_{x_{k}}^{\varepsilon_{k}}\right\}>0 .
$$

After extracting a subsequence, one can suppose $x_{k} \rightarrow x$ for some point $x \in \mathbb{P}(V)$. As $\nu_{\breve{\mu}}\left(\mathcal{Q}_{x}\right)=0$, there exists $\varepsilon>0$ such that $\nu_{\breve{\mu}}\left(\mathcal{Q}_{x}^{\varepsilon}\right)<\frac{1}{2} \rho$. Thus, if we set, for $\zeta$ in $\mathcal{P}_{\check{\mu}}$

$$
\varphi(\zeta)=\max \left(0,1-\frac{1}{\varepsilon} d\left(x, \mathbb{P}\left(\left(V_{\zeta}^{*}\right)^{\perp}\right)\right)\right),
$$

the non-negative continuous function $\varphi$ satisfies $\int_{\mathcal{P}_{\check{\mu}}} \varphi \mathrm{d} \nu_{\check{\mu}}<\frac{1}{2} \rho$. Now, on the one hand, by (4.8) we have

$$
\int_{B^{U}} \varphi\left(\xi_{b_{0}^{\prime} \cdots b_{n_{k}-1}^{\prime}}^{m}\right) \mathrm{d} \beta_{n_{k}, c}^{U}\left(b^{\prime}, z^{\prime}\right) \underset{k \rightarrow \infty}{\longrightarrow} \int_{\mathcal{P}_{\check{\mu}}} \varphi \mathrm{d} \nu_{\check{\mu}}<\frac{1}{2} \rho
$$

and on the other hand, by the triangle inequality, as soon as $k$ is large, $\mathcal{Q}_{x_{k}}^{\varepsilon_{k}} \subset \mathcal{Q}_{x}^{\varepsilon / 2}$. Hence $\varphi \geq \frac{1}{2}$ on $\mathcal{Q}_{x_{k}}^{\varepsilon_{k}}$ and

$$
\int_{B^{U}} \varphi\left(\xi_{b_{0}^{\prime} \cdots b_{n_{k}-1}^{\prime}}^{m}\right) \mathrm{d} \beta_{n_{k}, c}^{U}\left(b^{\prime}, z^{\prime}\right) \geq \frac{1}{2} \rho
$$

whence a contradiction. This proves (4.19).

We now prove (4.18). For any $W:=V_{\eta} \subset V$, set

$$
\mathcal{Q}_{W}:=\bigcup_{y \subset W} \mathcal{Q}_{y} \text { and } \mathcal{Q}_{W}^{\varepsilon}:=\bigcup_{y \subset W} \mathcal{Q}_{y}^{\varepsilon},
$$

where the unions are over the lines $y \in \mathbb{P}(V)$ included in $W$. As $W=V_{\eta}, \mathcal{Q}_{W}$ is a proper algebraic subvariety of $\mathcal{P}_{\breve{\mu}}$, and we still have $\nu_{\breve{\mu}}\left(\mathcal{Q}_{W}\right)=0$.

According to (4.3) in Lemma 4.1, to prove (4.18) it suffices to combine (4.19) with the fact that, for $\beta^{U}$-almost any $c=(b, z)$ in $B^{U}$,

$$
\lim _{\varepsilon \rightarrow 0} \limsup _{n \rightarrow \infty} \sup _{W \subset V} \beta_{n, c}^{U}\left\{\left(b^{\prime}, z^{\prime}\right) \in B^{U} \mid \xi_{b_{0}^{\prime} \cdots b_{n-1}^{\prime}}^{m} \in \mathcal{Q}_{W}^{\varepsilon}\right\}=0,
$$

where sup is over all the subspaces $W$ of the form $V_{\eta}$ for some $\eta$ in $\mathcal{P}_{\mu}$. The proof of (4.20) is exactly the same as that of (4.19).

\section{DyNAMiCs ON HOMOgENEOUS SPACES}

The first two sections of this chapter describe the class of $\mathcal{S}$-adic groups with which we will be working. The other sections contain a collection of technical facts on the dynamics on homogeneous spaces that we will use in the proof of Theorem 2.7.

We still let $\{\infty, 2,3,5, \ldots\}$ be the set of prime numbers including $\infty, \mathcal{S}$ be a finite subset of this set and $\mathbb{Q}_{\mathcal{S}}$ be the algebra $\prod_{p \in \mathcal{S}} \mathbb{Q}_{p}$. 


\subsection{Semiconnected groups.}

The reader who is mainly interested in real Lie groups may skip this section in which we describe the structure of $\mathcal{S}$-adic Lie groups. All these structure results are well known for real Lie groups.

Definition 5.1. An $\mathcal{S}$-adic Lie group $G$ is a locally compact group which contains an open subgroup $U$ isomorphic to a group of the form $\left(\prod_{p \in \mathcal{S}} G_{p}\right) / \Delta$ where, for each $p \in \mathcal{S}, G_{p}$ is a $p$-adic Lie group and $\Delta$ is a discrete normal subgroup of this product (see [3]).

Let $G$ be an $\mathcal{S}$-adic Lie group. We will denote by $\mathfrak{g}_{p}$ the Lie algebra of $G_{p}$ and by $\mathfrak{g}$ the Lie algebra of $G$ which is the direct sum of the Lie algebras $\mathfrak{g}_{p}$. We will consider $\mathfrak{g}$ as a $\mathbb{Q}_{\mathcal{S}}$-module. Then, any submodule of $\mathfrak{g}$ is closed and is a direct sum of $\mathbb{Q}_{p}$-vector subspaces of $\mathfrak{g}_{p}$. We set $\mathfrak{g}_{f}=\bigoplus_{p<\infty} \mathfrak{g}_{p}$ for the non-archimedean part of $\mathfrak{g}$, and we let $G_{\infty}$ be the connected analytic subgroup of $G$ with Lie algebra $\mathfrak{g}_{\infty}$. We will denote by $\operatorname{Ad}_{\mathfrak{g}}, \operatorname{Ad}_{\mathfrak{g}_{p}}$, etc., the adjoint action of $G$ on $\mathfrak{g}, \mathfrak{g}_{p}$, etc.

If $G^{\prime}$ is a closed subgroup of $G$, it is an $\mathcal{S}$-adic Lie subgroup and its Lie algebra is a submodule of $\mathfrak{g}$ (see [26, Prop. 1.5]).

We choose a good norm $\|$.$\| on each of the p$-adic Lie algebras $\mathfrak{g}_{p}$ and, for $v=$ $\left(v_{p}\right)_{p \in \mathcal{S}}$ in $\mathfrak{g}$, we set $\|v\|:=\max _{p \in \mathcal{S}}\left\|v_{p}\right\|$.

5.1.1. Exponential maps. When $G$ is not a real Lie group, one has to pay attention to the fact that exponential maps are only defined in a neighborhood of 0 and that they are not unique.

When $S=\{\infty\}$, we define a standard open set $\Omega$ of $G$ as being a relatively compact open neighborhood of $e$ which is the diffeomorphic image of some convex symmetric open neighborhood of 0 in $\mathfrak{g}$ by the exponential map.

When $\mathcal{S}=\{p\}$ for some finite $p$, we say that an element $g$ of $G$ admits a logarithm if one has $g^{p^{n}} \underset{n \rightarrow \infty}{\longrightarrow} e$ : indeed, for such a $g$, the map $n \mapsto g^{n}$ extends as a continuous morphism $\mathbb{Z}_{p} \rightarrow G$ and one can define the logarithm log $g$ of $g$ as being the derivative at 0 of this morphism. Now, $G$ is said to be a standard group if there exists a $\mathbb{Q}_{p}$-Lie algebra $\mathfrak{l}$ and a compact open sub- $\mathbb{Z}_{p}$-algebra $O$ of $\mathfrak{l}$ such that the Baker-Campbell-Hausdorff series converges on $O$ and $G$ is isomorphic to the $p$-adic Lie group $O$, equipped with the group law defined by this formula. In this case, $\mathfrak{l}$ identifies canonically with $\mathfrak{g}$, every element of $G$ admits a logarithm and the logarithm map induces an isomorphism $G \rightarrow O$. If $G$ is any $p$-adic Lie group, it admits a standard open subgroup (see [16, Theorem 8.29]). If $\Omega$ is such a subgroup and if $O$ is the associated compact open sub- $\mathbb{Z}_{p}$-algebra of $\mathfrak{g}$, we denote by $\exp _{\Omega}: O \rightarrow \Omega$ the inverse of the logarithm map $\Omega \rightarrow O$.

In general, a standard open subset $\Omega$ of $G$ is an open neighborhood of $e$ of the form $\Omega=\prod_{p \in \mathcal{S}} \Omega_{p}$, where $\Omega_{\infty}$ is a standard open subset of $G_{\infty}$ and, for any $p \neq \infty$ in $\mathcal{S}, \Omega_{p}$ is a standard $p$-adic Lie group embedded in $G$ such that $\Omega_{\infty} \Omega_{\infty} \cap \prod_{p<\infty} \Omega_{p}=\{e\}$. We then define the exponential map $\exp _{\Omega}$ as the product of the exponential maps of $\Omega_{p}, p \in \mathcal{S}$. This is a diffeomorphism from a relatively compact open subset $O=\prod_{p \in \mathcal{S}} O_{p}$ of $\mathfrak{g}$ onto $\Omega$. The standard open subsets of $G$ form a basis of neighborhoods of $e$ in $G$. We set $\Omega_{f}=\prod_{p<\infty} \Omega_{p}$.

Note that if $\Omega$ and $\Omega^{\prime}$ are standard open subsets of $G$, the maps $\exp _{\Omega}$ and $\exp _{\Omega^{\prime}}$ coincide in some neighborhood of 0 in $\mathfrak{g}$. 
We will need our exponential map to enjoy a nice equivariant property under the adjoint action. This equivariance property is automatic for a real Lie group since the exponential map is uniquely defined.

Lemma 5.2. Let $G$ be an $\mathcal{S}$-adic Lie group, $\Omega \subset G$ a standard open set and $\exp _{\Omega}$ : $O \rightarrow \Omega$ the corresponding exponential map. For every compact subset $K \subset G$, there exists an open subset $O_{K}$ which is contained in $O$ and in all the translates $\operatorname{Ad} g^{-1}(O), g \in K$, and such that one has the equivariance property

$$
\exp _{\Omega}(\operatorname{Ad} g(v))=g \exp _{\Omega}(v) g^{-1} \quad \text { for any } v \in O_{K}, g \in K \text {. }
$$

Proof. We may assume that $K$ contains $e$. The intersection $\Omega_{K}:=\bigcap_{g \in K} g^{-1} \Omega g$ is an open neighborhood of $e$ in $G$. We just choose $O_{K}$ to be the open set $O_{K}:=$ $\log \left(\Omega_{K}\right)$.

5.1.2. Unipotent one-parameter subgroups. Assume now $G$ is a closed subgroup of a product, for $p$ in $\mathcal{S}$, of $p$-adic Lie groups. A one-parameter subgroup $\varphi$ of $G$, i.e. a continuous morphism $\varphi: \mathbb{Q}_{p} \rightarrow G ; t \mapsto \varphi(t)$ for some $p \in \mathcal{S}$, is said to be Ad-unipotent if, for any $t$ in $\mathbb{Q}_{p}, \varphi(t)$ belongs to the $p$-adic factor of the product and $\operatorname{Ad}_{\mathfrak{g}_{p}}(\varphi(t))$ is a unipotent automorphism of $\mathfrak{g}_{p}$. Its derivative is a nilpotent derivation of $\mathfrak{g}_{p}$. When $G$ is a $p$-adic Lie group with $p$ finite, every one-parameter subgroup $\varphi: \mathbb{Q}_{p} \rightarrow G ; t \mapsto \varphi_{t}$ is Ad-unipotent (see [26, Corollary 1.2]).

Remark 5.3. This definition is the one used by Ratner in 26]. It actually depends on the closed embedding of $G$ in a product of $p$-adic Lie groups (think for example to the group $\mathbb{Q}_{p} \times(\mathbb{R} / \mathbb{Z}), p<\infty$, which admits automorphisms that do not preserve $\mathbb{Q}_{p}$ ). It would perhaps be more comfortable to work with one-parameter subgroups $\varphi: \mathbb{Q}_{p} \rightarrow G$ such that, for any $t, \operatorname{Ad}_{\mathfrak{g}_{p}}(\varphi(t))$ is a unipotent automorphism of $\mathfrak{g}_{p}$ and, for $q \neq p, \operatorname{Ad}_{\mathfrak{g}_{q}}(\varphi(t))=0$. But there does not yet exist a published reference of Ratner's Theorem 5.15 for subgroups spanned by such one-parameter subgroups. The reader will note that all the one-parameter subgroups which will be constructed in this paper are Ad-unipotent for any closed embedding of $G$ in a product of $p$-adic Lie groups (see Lemma 5.4 below and Lemma 5.12).

Let us give a construction of Ad-unipotent one-parameter subgroups of $G$.

Lemma 5.4 (Construction of unipotent subgroups). Let $G$ be an $\mathcal{S}$-adic Lie group, $G^{\prime}$ be a normal closed subgroup of $G$ with Lie algebra $\mathfrak{g}^{\prime}, g$ be an element of $G$, $p \in \mathcal{S}$ and $V \subset \mathfrak{g}_{p}^{\prime}$ denote the sum of the generalized eigenspaces of $\operatorname{Ad}_{\mathfrak{g}_{p}^{\prime}}$ which are associated to eigenvalues with modulus $<1$. Then, there exists a unique $\mathrm{Ad} g$ equivariant map $\varphi: V \rightarrow G^{\prime}$ such that, for any $v$ in $V$, the map $\mathbb{Q}_{p} \rightarrow G ; t \mapsto$ $\varphi(t v)$ is a one-parameter subgroup and that $\varphi$ is equal to an exponential map in the neighborhood of 0 . If $G$ is a closed subgroup of a product of p-adic Lie groups, $p \in \mathcal{S}$, for any $v$ in $V$, the one-parameter subgroup $t \mapsto \varphi(t v)$ is Ad-unipotent.

Proof. Let us construct this map. We will write $g$ for $\operatorname{Ad} g$. We can assume $p<\infty$, the real case being trivial. Let $\Omega$ be a standard open subset of $G$ with exponential map $\exp _{\Omega}: O \rightarrow \Omega$. We assume $\exp _{\Omega}\left(O \cap \mathfrak{g}^{\prime}\right) \subset G^{\prime}$. By Lemma 5.2, there exists an open subgroup $U \subset O_{p} \cap V$ such that $g U \subset O_{p}$ and that $\exp _{\Omega}(u)=g^{-1} \exp _{\Omega}(g u) g$ for any $u$ in $U$. After eventually replacing $U$ by $\bigcap_{k>0} g^{-k} U$, we can assume $g U \subset U$. Now, for $k$ in $\mathbb{Z}$, let $U_{k}=g^{-k} U$ and define a continuous map $\varphi_{k}: U_{k} \rightarrow G^{\prime}$ by setting $\varphi_{k}(u)=g^{-k} \exp _{\Omega}\left(g^{k} u\right) g^{k}$ for any $u$ in $U_{k}$. We claim that, for any $k$, one 
has $\varphi_{k}=\varphi_{k-1}$ on $U_{k-1}=g U_{k}$. Indeed, let $u$ be in $U_{k}$. As $g^{k} u$ belongs to $U$, we have

$$
\varphi_{k+1}(u)=g^{-k}\left(g^{-1} \exp _{\Omega}\left(g g^{k} u\right) g\right) g^{k}=g^{-k} \exp _{\Omega}\left(g^{k} u\right) g^{k}=\varphi_{k}(u) .
$$

Therefore, as $V=\bigcup_{k \in \mathbb{Z}} U_{k}$, the map $\varphi: V \rightarrow G^{\prime}$ whose restriction to any $U_{k}$, $k \in \mathbb{Z}$, is $\varphi_{k}$ satisfies our requirements. Uniqueness is clear.

Now, note that, by uniqueness, if $G$ is a closed subgroup of a product $\Pi_{q \in \mathcal{S}} H_{q}$, where, for any $q$ in $\mathcal{S}, H_{q}$ is a $q$-adic Lie group, we have $\varphi(V) \subset H_{p}$. Besides, for any $v$ in $V$, as $g^{k} v \underset{k \rightarrow \infty}{\longrightarrow} 0$, one has

$$
\left(\operatorname{Ad} g^{k}\right)(\operatorname{ad} v)\left(\operatorname{Ad} g^{-k}\right) \underset{k \rightarrow \infty}{\longrightarrow} 0
$$

and $\operatorname{ad} v$ is a nilpotent endomorphism of $\mathfrak{g}$. Again by uniqueness, we have $\operatorname{Ad}(\varphi(v))$ $=\exp (\operatorname{ad} v)$ and the result follows.

5.1.3. Semiconnected components. We now introduce the notion of a semiconnected component (see [26, p. 288]). For real Lie groups, a semiconnected component is nothing but the connected component.

Assume $G$ is a closed subgroup of a product, for $p$ in $\mathcal{S}$, of $p$-adic Lie groups. Let $\Omega$ be a standard open set in $G$. For any closed subgroup $G^{\prime}$ of $G$, we denote by $G_{\Omega}^{\prime}$ the intersection $G_{\Omega}^{\prime}:=G^{\prime} \cap \Omega_{f}$, by $G_{u}^{\prime}$ the subgroup of $G^{\prime}$ generated by all the one-parameter Ad-unipotent subgroups of $G$ which are contained in $G^{\prime}$ and by $\mathfrak{g}_{u}^{\prime}$ the Lie algebra of $G_{u}^{\prime}$. We denote by $G_{\infty}^{\prime}$ the real Lie subgroup of $G^{\prime}$ with Lie algebra $\mathfrak{g}_{\infty}^{\prime}$. This group $G_{\infty}^{\prime}$ is also equal to the connected component of $G^{\prime}$. The groups $G_{u}^{\prime}$ and $G_{\infty}^{\prime}$ are normal in $G^{\prime}$.

Definition 5.5 ([26]). The $\Omega$-semiconnected component of $G^{\prime}$ is the subgroup $G_{\Omega}^{\prime} G_{u}^{\prime} G_{\infty}^{\prime}$ of $G^{\prime}$

The subgroup $G^{\prime}$ is said to be semiconnected if $G^{\prime}=G_{\Omega}^{\prime} G_{u}^{\prime} G_{\infty}^{\prime}$ for some standard open subset $\Omega$ of $G$. A semiconnected component of $G^{\prime}$ is a semiconnected open subgroup of $G^{\prime}$ which contains $G_{u}^{\prime}$.

When $\mathcal{S}=\{\infty\}$, if a subgroup $\Gamma$ normalizes a closed subgroup $G^{\prime}$ of $G$, then it normalizes its connected component. In general, we will need an analogue property to hold under a suitable assumption on $\Gamma$. Recall that $\overline{\operatorname{Ad}(\Gamma)}^{Z}$ denotes the Zariski closure of $\operatorname{Ad} \Gamma$ in $\operatorname{GL}(\mathfrak{g})$, that is, the product of the Zariski closures of the $\operatorname{Ad}_{\mathfrak{g}_{p}}(\Gamma)$ in the $\operatorname{GL}\left(\mathfrak{g}_{p}\right), p \in \mathcal{S}$, and that it is said to be semisimple if all the groups $\frac{\operatorname{Ad}_{\mathfrak{g}_{p} \Gamma}}{}$, $p \in \mathcal{S}$, are semisimple.

Lemma 5.6 (Invariant semiconnected component). Let $G$ be an $\mathcal{S}$-adic Lie group that is a closed subgroup of a product, for $p$ in $\mathcal{S}$, of $p$-adic Lie groups. Let $\Gamma$ be a compactly generated subgroup of $G$ such that $\overline{\operatorname{Ad}(\Gamma)}$ is semisimple. Then, if $G^{\prime}$ is a closed subgroup of $G$ which is normalized by $\Gamma$, there exist arbitrarily small standard open subsets $\Omega$ of $G$ such that $\Gamma$ normalizes an $\Omega$-semiconnected open subgroup of $G^{\prime}$.

Proof. Let $\mathfrak{g}^{\prime}$ be the Lie algebra of $G^{\prime}$ and $\mathfrak{l} \subset \mathfrak{g}^{\prime}$ be the subspace of those $v$ in $\mathfrak{g}^{\prime}$ whose $A d \Gamma$-orbit is bounded. Then $\mathfrak{l}$ is a subalgebra of $\mathfrak{g}^{\prime}$ and the group $\overline{\operatorname{Ad}_{\mathfrak{l}} \Gamma}$ is compact. As $\Gamma$ is compactly generated, by Lemma 5.2, there exists an arbitrarily small standard open subset $\Omega$ of $G$ with exponential map $\exp _{\Omega}: O \rightarrow \Omega$ such that $O \cap \mathfrak{l}$ is $A d \Gamma$-invariant and that, for any $v$ in $O \cap \mathfrak{l}$ and $g$ in $\Gamma$, one has 
$\exp _{\Omega}(g v)=g \exp _{\Omega}(v) g^{-1}$. In particular, if $L_{\infty}$ is the connected Lie subgroup of $G$ with Lie algebra $\mathfrak{l}_{\infty}$, the group $\exp _{\Omega}\left(O \cap \mathfrak{l}_{f}\right) L_{\infty}$ is normalized by $\Gamma$ and has Lie algebra $\mathfrak{l}$.

Since the group $\overline{\operatorname{Ad}(\Gamma)}^{Z}$ is semisimple, $\mathfrak{l}$ admits a (unique) $\Gamma$-invariant complementary submodule $\mathfrak{v}$ in $\mathfrak{g}^{\prime}$. As the $\Gamma$-orbits of non-zero vectors in $\mathfrak{v}$ are unbounded, the module $\mathfrak{v}$ is spanned by submodules $V$ satisfying the assumptions of Lemma 5.4 for some $g$ in $\Gamma$ (see [2]). Therefore, by this lemma, the Lie algebra of the group $G_{u}^{\prime}$ contains $\mathfrak{v}$. In other words, the $\Omega$-semiconnected group $G^{\prime \prime}=\exp _{\Omega}\left(O \cap \mathfrak{l}_{f}\right) L_{\infty} G_{u}^{\prime}$ is normalized by $\Gamma$.

Recall that weakly regular $\mathcal{S}$-adic Lie groups have been defined in Definition 2.1. We will need to relate this notion to the one of regular $\mathcal{S}$-adic Lie groups.

Definition 5.7 (Ratner, [26]). If $p<\infty$, a $p$-adic Lie group $G$ is said to be regular if the kernel of the adjoint map $\operatorname{Ker}\left(\operatorname{Ad}_{\mathfrak{g}}\right)$ is equal to the center $Z(G)$ of $G$ and if the finite subgroups of $G$ have uniformly bounded order.

If $p=\infty$, every real Lie group is said to be regular.

An $\mathcal{S}$-adic Lie group is said to be regular if it is isomorphic to a closed subgroup of a product of regular $p$-adic Lie groups.

According to [26, Cor. 1.3], regular groups are weakly regular. The following proposition is a kind of converse that will be useful to extend the level of generality of Ratner's Theorem 5.15.

Proposition 5.8. Let $G$ be a weakly regular semiconnected p-adic Lie group. Then $G$ contains an open finite index subgroup which is regular.

Proposition 5.8 will be proved in $[10$.

\subsection{Good exponentials.}

In this section we define a class of groups which will play an important role in our proof: the groups with "good exponentials". The reader who is only interested in real Lie groups may skip the beginning of this section and proceed to Proposition 5.13

We will need a local exponential map on $G$ with stronger equivariance properties than in Lemma 5.2 .

Definition 5.9. Let $G$ be an $\mathcal{S}$-adic Lie group and $\Gamma \subset G$ a subgroup. We say that $(G, \Gamma)$ has good exponentials if there exists a standard open subset $\Omega$ of $G$ with exponential map $\exp _{\Omega}: O \rightarrow \Omega$ satisfying the following equivariance property: for any $v \in O$ and $g \in \Gamma$ such that $\operatorname{Ad} g(v) \in O$, one has

$$
\exp _{\Omega}(\operatorname{Ad} g(v))=g \exp _{\Omega}(v) g^{-1} .
$$

Such an exponential map $\exp _{\Omega}$ will be called a $(G, \Gamma)$-good exponential map.

Example 5.10. Such an open set $\Omega$ may not exist when $G$ is not weakly regular, even if this group $G$ is compactly generated. For instance, it does not exist when $G=\Gamma$ is the amalgamated product of two copies of $\operatorname{SL}\left(2, \mathbb{Q}_{p}\right)$ over the open subgroup $\mathrm{SL}\left(2, \mathbb{Z}_{p}\right)$. 
Good exponential maps often exist:

Proposition 5.11 (Good exponential maps). Let $G$ be an $\mathcal{S}$-adic Lie group and $\Gamma \subset G$ a subgroup such that $\overline{\mathrm{Ad}(\Gamma)}^{Z}$ is semisimple.

a) Assume that $(i): G$ is a real Lie group, or $(i i): G$ is a closed subgroup of $\prod_{p \in \mathcal{S}} \mathrm{GL}\left(d_{p}, \mathbb{Q}_{p}\right)$, or $($ iii $): G$ is weakly regular and $\Gamma$ is compactly generated. Then $(G, \Gamma)$ has good exponentials.

b) Let $G^{\prime}$ be a closed subgroup of $G$ containing $\Gamma$. If $(G, \Gamma)$ has good exponentials, then $\left(G^{\prime}, \Gamma\right)$ has good exponentials.

c) Let $S$ be a closed normal subgroup of $G, \underline{G}:=G / S$ and $\underline{\Gamma}:=\Gamma S / S$. If $(G, \Gamma)$ has good exponentials, then $(\underline{G}, \underline{\Gamma})$ has good exponentials.

Proof. a) (i) When $G$ is real, there exists only one exponential map.

(ii) One uses the usual series for the exponential of matrices.

(iii) By Lemma 5.6, there exists a standard open subset $\Omega$ of $G$, with exponential map $\exp _{\Omega}: O \rightarrow \Omega$, such that some $\Omega$-semiconnected open subgroup $G^{\prime}$ of $G$ is normalized by $\Gamma$. By Proposition 5.8 , after eventually shrinking $\Omega$, we may assume $G^{\prime}$ is regular. We can also assume, for any $v$ in $O$, that the matrix exponential series converges at ad $v$ and

$$
\operatorname{Ad}\left(\exp _{\Omega} v\right)=\exp (\operatorname{ad} v) .
$$

Since $G^{\prime}$ is regular, the order of the finite subgroups of $G^{\prime}$ is bounded by some constant $n$. We set $O^{\prime}=(n !) O$ and $\Omega^{\prime}=\exp _{\Omega}\left(O^{\prime}\right)$ and we claim that $\exp _{\Omega^{\prime}}=$ $\left.\exp _{\Omega}\right|_{\Omega^{\prime}}$ is $(G, \Gamma)$-good. Indeed, let $v$ be in $O^{\prime}$ and $g$ be in $\Gamma$ with $g v \in O^{\prime}$. We set $w=\frac{1}{n !} v$ so that $w, g w \in O$. Let $h=g \exp _{\Omega}(w) g^{-1} \exp _{\Omega}(g w)^{-1}$. By (5.2), we get $\operatorname{Ad} h=e$; hence, $G^{\prime}$ being regular, $h$ is a central element of $G^{\prime}$. Now, there exists an integer $m$ such that $\exp _{\Omega}(m g w)=g \exp _{\Omega}(m w) g^{-1}$ so that $h^{m}=e$ and hence $h^{n !}=e$, that is, precisely $\exp _{\Omega}(g v)=g \exp _{\Omega}(v) g^{-1}$, which should be proved.

b) This is clear.

c) We write $\mathfrak{g}=\mathfrak{s} \oplus \mathfrak{t}$, where $\mathfrak{t}$ is a $\Gamma$-invariant complementary subspace of the Lie algebra $\mathfrak{s}$ of $S$ in $\mathfrak{g}$ and we introduce the projection $p: G \rightarrow \underline{G}$. We fix a standard open subset $\Omega$ of $G$ whose exponential map $\exp _{\Omega}: O \rightarrow \Omega$ is $(G, \Gamma)$-good. We identify the Lie algebra of $\underline{G}$ with $\mathfrak{t}$ and set $\underline{O}:=O \cap \mathfrak{t}$. The good exponential map $\exp _{\underline{\Omega}}: \underline{O} \rightarrow \underline{G}$ is then defined, for $v \in \underline{O}$, by $\exp _{\underline{\Omega}}(v):=p\left(\exp _{\Omega}(v)\right)$.

If $\Gamma$ is a subgroup of $G$, we introduce the closed cone of $\Gamma$-unstable elements of the Lie algebra $\mathfrak{g}$,

$$
N_{\Gamma}(\mathfrak{g}):=\left\{v \in \mathfrak{g} \mid \exists\left(g_{n}\right) \subset \Gamma \quad \operatorname{Ad} g_{n}(v) \underset{n \rightarrow \infty}{\longrightarrow} 0\right\} .
$$

Its elements are ad-nilpotent elements of $\mathfrak{g}$. When $(G, \Gamma)$ has good exponentials, we can extend the construction in Lemma 5.4.

Lemma 5.12 (Construction of unipotent subgroups (bis)). Let $G$ be an $\mathcal{S}$-adic Lie group and $\Gamma \subset G$ a subgroup of $G$. Assume that $(G, \Gamma)$ has good exponentials. Then there exists a unique map $\exp _{N}: N_{\Gamma}(\mathfrak{g}) \longrightarrow G$ satisfying the following equivariance and compatibility properties: for any $v \in N_{\Gamma}(\mathfrak{g}), g \in \Gamma$, one has

$$
\exp _{N}(\operatorname{Ad} g(v))=g \exp _{N}(v) g^{-1},
$$

and for any good exponential map $\exp _{\Omega}: O \rightarrow \Omega$, any $v \in O \cap N_{\Gamma}(\mathfrak{g})$, one has

$$
\exp _{N}(v)=\exp _{\Omega}(v) \text {. }
$$


If $G$ is a closed subgroup of a product of $p$-adic Lie groups, $p \in \mathcal{S}$, for any $v$ in $V$, the one-parameter subgroup $t \mapsto \varphi(t v)$ is Ad-unipotent.

Proof. For any $v$ in $N_{\Gamma}(\mathfrak{g})$, there exists $g \in \Gamma$ such that $\operatorname{Ad}^{-1}(v) \in O$. We then set

$$
\exp _{N}(v)=g \exp _{\Omega}\left(\operatorname{Ad} g^{-1}(v)\right) g^{-1} .
$$

This definition does not depend on the choice of $g$ because of the equivariance property (5.1). By construction, it satisfies the equivariance property (5.4) and the compatibility condition (5.5). Uniqueness is obtained in the same way.

Now, if $v$ belongs to $N_{\Gamma}(\mathfrak{g}) \cap \mathfrak{g}_{p}$ for some $p$ in $\mathcal{S}$, by uniqueness we get $\operatorname{Ad}\left(\exp _{N}(v)\right)$ $=\exp (\operatorname{ad}(v))$, and then $\operatorname{Ad}\left(\exp _{N}(v)\right)$ is unipotent.

To shorten notation, when there is no ambiguity, we will write $e^{v}$ or $\exp (v)$ for $\exp _{\Omega}(v)$ or for $\exp _{N}(v)$.

Let us use these notions to proceed to a construction that will play a key role in many of our proofs. Recall that if $\mu$ is a probability measure on $G$, we let $\Gamma_{\mu}$ denote the subgroup of $G$ spanned by the support of $\mu$ and we say $\mu$ is Ad-semisimple if the group $H_{\mu}={\overline{\mathrm{Ad}} \Gamma_{\mu}}^{Z}$ is semisimple. In this case, we may freely use the notation and results from sections 4.2 and 4.3 for the probability measure $\operatorname{Ad}_{*} \mu$ on $H_{\mu}$. Thus, we get a map $\xi: B \rightarrow \mathcal{P}_{\mu}$ and, for any $H_{\mu}$-irreducible subrepresentation $\mathfrak{v}$ of $\mathfrak{g}$, we get an $H_{\mu}$-equivariant map $\mathcal{P}_{\mu} \rightarrow \operatorname{Gr}(\mathfrak{v}) ; \eta \mapsto \mathfrak{v}_{\eta}$. When $\mathfrak{v}$ is not irreducible, we set, for $\eta$ in $\mathcal{P}_{\mu}, \mathfrak{v}_{\eta}=\sum_{\mathfrak{w}} \mathfrak{w}_{\eta}$, where $\mathfrak{w}$ varies among the $H_{\mu}$-irreducible subrepresentations of $\mathfrak{v}$. This is the space of invariant vectors for the action in $\mathfrak{v}$ of the unipotent radical of the stabilizer of $\eta$ in $H_{\mu}$. We set $\mathfrak{v}_{b}=\mathfrak{v}_{\xi_{b}}$ for $\beta$-almost every $b$ in $B$.

Proposition 5.13 (Construction of $\left.V_{b}\right)$. Let $G$ be an $\mathcal{S}$-adic Lie group and $\mu$ be an Ad-semisimple Borel probability measure on $G$ such that $\left(G, \Gamma_{\mu}\right)$ has good exponentials and $\int_{G} \log \|\operatorname{Ad} g\| \mathrm{d} \mu(g)<\infty$. Let $\mathfrak{g}^{\prime}$ be a subalgebra of $\mathfrak{g}$ which is $H_{\mu}$-invariant. We set $\mathfrak{v}$ to be the sum of the irreducible sub- $H_{\mu}$-representations of $\mathfrak{g}^{\prime}$ in which the image of $\Gamma_{\mu}$ is unbounded. The following properties hold:

a) For any $\eta$ in $\mathcal{P}_{\mu}, \mathfrak{v}_{\eta}$ is an ad-nilpotent subalgebra of $\mathfrak{g}^{\prime}$.

b) For $\beta$-almost any $b$ in $B$, one has $\mathfrak{v}_{b}=\operatorname{Ad} b_{0} \mathfrak{v}_{T b}$ and the norm of the restriction of $\operatorname{Ad}\left(b_{0} \cdots b_{n-1}\right)^{-1}$ to $\mathfrak{v}_{b}$ goes to 0 as $n \rightarrow \infty$. In particular, $\mathfrak{v}_{b} \subset N_{\Gamma_{\mu}}(\mathfrak{g})$.

c) For $\beta$-almost any $b$ in $B$, the set $V_{b}:=\exp _{N}\left(\mathfrak{v}_{b}\right)$ is a Lie subgroup of $G$ and $V_{b}=b_{0} V_{T b} b_{0}^{-1}$.

Remark 5.14. Define a $\mathbb{Q}_{\mathcal{S}}$-unipotent group to be a product of $\mathbb{Q}_{p}$-unipotent groups for $p$ in $\mathcal{S}$. Then, one could prove that, for $\beta$-almost any $b$ in $B, V_{b}$ is isomorphic to the unique $\mathbb{Q}_{\mathcal{S}}$-unipotent group with Lie algebra $\mathfrak{v}_{b}$. We shall not use this fact.

Proof of Proposition 5.13, a) It suffices to prove this fact when $\eta=\eta_{\Theta_{\mu}}$, the fixed point of $P_{\mu}$ in $\mathcal{P}_{\mu}$. We set $\mathfrak{v}_{0}=\mathfrak{v}_{\eta_{\Theta}}$. By construction, $\mathfrak{v}_{0}$ is the $\mathbb{Q}_{\mathcal{S}}$-module spanned by the set $E$ of $U_{\mu}$-invariant vectors $v$ in $\mathfrak{g}^{\prime}$ such that there exists a dominant weight $\chi$ of $A$ whose restriction to $A_{\mu}$ is non-trivial and satisfies $a v=\chi(a) v$ for any $a$ in $A_{\mu}$. Now, as $H_{\mu}$ is semisimple, if $\chi$ and $\chi^{\prime}$ are dominant weights of $A$ who have non-trivial restriction to $A_{\mu}$, so does $\chi \chi^{\prime}$. Therefore, for any $v, v^{\prime}$ in $E$, we have $\left[v, v^{\prime}\right] \in E$ and $\mathfrak{v}_{0}$ is a Lie algebra.

b) The equivariance property follows from the one of the map $\xi$. Since $\mu$ has a finite first order moment, by Birkhoff's ergodic theorem and Proposition 4.4. for $\beta$-almost any $b$ in $B$, for any dominant weight $\chi$ of $A$ whose restriction to $A_{\mu}$ is not 
trivial, we have $\chi^{\omega}\left(\theta_{\mathbb{R}, n}(b)\right) \underset{n \rightarrow \infty}{\longrightarrow} \infty$. Now, the result follows from the definition of $\mathfrak{v}_{b}$ and Lemma 4.2 .

c) The equivariance property of $V_{b}$ is a consequence of the ones of $\mathfrak{v}_{b}$ and of $\exp _{N}$. In order to check that $V_{b}$ is a subgroup of $G$, we write, for $\beta$-almost any $b$ in $B, \mathfrak{v}_{b}=\bigoplus_{p \in \mathcal{S}} \mathfrak{v}_{p, b}$ as the decomposition of $\mathfrak{v}_{b}$ into $p$-adic components and set, for $p$ in $\mathcal{S}, V_{p, b}=\exp _{N}\left(\mathfrak{v}_{p, b}\right)$. As these sets commute with each other, it suffices to prove that each $V_{p, b}$ is a subgroup.

If $p=\infty$, then $V_{\infty, b}$ is the connected Lie subgroup of $G$ with Lie algebra $\mathfrak{v}_{\infty, b}$, and the result follows from the structure theory of real nilpotent Lie groups.

If $p<\infty$, we will prove that $V_{p, b}$ is an increasing union of compact open subgroups. Let $\Omega$ be a $\left(G, \Gamma_{\mu}\right)$-good standard open subset of $G$, with exponential map $\exp _{\Omega}: O \rightarrow \Omega$. For any subalgebra $\mathfrak{l}$ of $\mathfrak{g}_{p}, \exp _{\Omega}(O \cap \mathfrak{l})$ is a compact subgroup of $G$. Now, for $\beta$-almost any $b$ in $B$, there exists a sequence $\left(n_{k}\right)$ of natural integers such that the sequence $b_{0} \cdots b_{n_{k}-1}\left(O \cap \mathfrak{v}_{p, T^{n_{k}}}\right)$ is an increasing sequence of compact subalgebras which exhausts $\mathfrak{v}_{b}$. Hence, by the equivariance property of $\exp _{N}$, the sequence

$$
\exp _{N}\left(b_{0} \cdots b_{n_{k}-1}\left(O \cap \mathfrak{v}_{p, T^{n_{k} b}}\right)\right)=b_{0} \cdots b_{n_{k}-1} \exp _{N}\left(O \cap \mathfrak{v}_{p, T^{n_{k} b}}\right)\left(b_{0} \cdots b_{n_{k}-1}\right)^{-1}
$$

is an increasing sequence of compact subgroups which exhausts $V_{b}$.

\subsection{Ratner's measures.}

In this section we recall the classification of measures which are invariant under groups spanned by Ad-unipotent one-parameter subgroups due to Ratner.

We keep the notation of sections 2.1 and 5.1. Let $G$ be a weakly regular $\mathcal{S}$-adic Lie group, $\Lambda$ a discrete subgroup of $G$ and $X=G / \Lambda$.

For a Borel probability measure $\alpha$ on $X$, we set $G_{\alpha}$ to be the stabilizer of $\alpha$ in $G$ and hence $G_{\alpha, u}$ to be the subgroup of $G_{\alpha}$ generated by the one-parameter Ad-unipotent subgroups of $G_{\alpha}$. We introduce the set $\mathcal{E}$ of Ratner's probability measures:

$$
\mathcal{E}:=\left\{\alpha \in \mathcal{H} \mid \operatorname{supp}(\alpha) \text { is a } G_{\alpha} \text {-orbit and } \alpha \text { is } G_{\alpha, u} \text {-ergodic }\right\} .
$$

We have Ratner's Theorem:

Theorem 5.15 (Ratner [26]). Let $G$ be a weakly regular $\mathcal{S}$-adic Lie group, $\Lambda$ be a discrete subgroup of $G$ and $H$ be a subgroup of $G$ such that $H=H_{u}$. Any $H$-invariant $H$-ergodic probability measure on $X=G / \Lambda$ belongs to $\mathcal{E}$.

More precisely, Ratner has proved this theorem when the $\mathcal{S}$-adic Lie group $G$ is regular. It can be extended to any weakly regular $\mathcal{S}$-adic Lie group thanks to our Proposition 5.8

\subsection{Fixed points and orbits of the centralizer.}

The following general lemma compares the set of fixed points of a subgroup in a homogeneous space and the orbits of its centralizer.

We will use this lemma to prove Lemma 7.9

Lemma 5.16. Let $G$ be a second countable $\mathcal{S}$-adic Lie group, $\Lambda, \Delta \subset G$ discrete subgroups of $G$ and $C$ the centralizer of $\Delta$ in $G$. We assume that $G$ is a real Lie group or that the group $\Delta$ is finitely generated. Then the set $X^{\Delta}$ of fixed points of 
$\Delta$ in $X=G / \Lambda$ is a countable union of $C$-orbits. Moreover, these orbits are open and closed in $X^{\Delta}$.

Proof of Lemma 5.16, Our assumptions ensure that the centralizer $\mathfrak{c}$ of $\Delta$ in $\mathfrak{g}$ is the Lie algebra of $C$. Since $\Delta$ and $C$ commute, the set $X^{\Delta}$ is a union of $C$ orbits. The main point is to prove that, for every $x$ in $X^{\Delta}$, the $C$-orbit $C x$ is a neighborhood of $x$ in $X^{\Delta}$. We have to find a small open neighborhood $U$ of $e$ in $G$ such that $U x \cap X^{\Delta} \subset C x$.

We pick a finite subset $F$ of $\Delta$ such that, in the space of endomorphisms of $\mathfrak{g}$, $\operatorname{Ad} F$ spans the same linear subspace as $\operatorname{Ad} \Delta$. Thus, $\mathfrak{c}$ is the centralizer of $F$ in $\mathfrak{g}$. By Lemma [5.2 we can choose a standard open subset $\Omega$ of $G$ with exponential map $\exp _{\Omega}: O \rightarrow \Omega$ such that, for any $v$ in $O$ and $f$ in $F$ with $f v \in O$, one has $\exp _{\Omega}(f v)=f \exp _{\Omega}(v) f^{-1}$, that $\exp _{\Omega}(O \cap \mathfrak{c}) \subset C$ and that the natural map $\Omega \rightarrow X ; g \mapsto g x$ is one-to-one. Set $O^{\prime}=O \cap \bigcap_{f \in F} f^{-1} O$. For any $v$ in $O^{\prime}$, if $\exp _{\Omega}(v) x$ is in $X^{\Delta}$, one has $v=f v$ for all $f \in F$. Hence $v$ is in $\mathfrak{c}$ and one has $\exp _{\Omega}\left(O^{\prime}\right) x \cap X^{\Delta} \subset C x$, as required.

This proves that the $C$-orbits are open in $X^{\Delta}$. Hence they are also closed and, since $X$ is second countable, there are only countably many $C$-orbits in $X^{\Delta}$.

\subsection{Stationary probability measures on homogeneous spaces.}

In the course of the proof of Theorem 2.7 which describes the stationary probability measures on $G / \Lambda$, we will also need to understand stationary probability measures on various $G$-spaces.

Let $V$ be a finite-dimensional $\mathbb{Q}_{\mathcal{S}}$-module, that is, a product $\prod_{p \in \mathcal{S}} V_{p}$ of finite dimensional vector spaces $V_{p}$ over $\mathbb{Q}_{p}, p \in \mathcal{S}$, and let $Y$ be the set of Radon measures $\lambda_{W}$ on $V$ which are a Haar measure on some closed $\mathbb{Q}_{\mathcal{S}}$-submodule $W=$ $\prod_{p \in \mathcal{S}} W_{p} \subset V$.

For any subgroup $\Gamma$ of $\operatorname{GL}(V)=\prod_{p} \operatorname{GL}\left(V_{p}\right)$, we set $\bar{\Gamma}^{Z}$ for the Zariski closure of $\Gamma$ and $\bar{\Gamma}^{Z, n c}$ for the intersection of the kernels of the algebraic representations $\rho: \bar{\Gamma}^{Z} \rightarrow \mathrm{GL}\left(d, \mathbb{Q}_{\mathcal{S}}\right)$ for which $\rho(\Gamma)$ is bounded.

Let $\mu$ be a probability measure on $\operatorname{GL}(V)$ and recall that $\Gamma_{\mu}$ denotes the closed subgroup generated by the support of $\mu$.

Lemma 5.17 (Stationary measures in $V$ ). Let $\mu$ be a probability measure on $\prod_{p} \mathrm{GL}\left(V_{p}\right)$ and $V^{\Gamma_{\mu}}$ and $Y^{\Gamma_{\mu}}$ be the sets of $\Gamma_{\mu}$-invariant elements in $V$ and $Y$, respectively.

We assume ${\overline{\Gamma_{\mu}}}^{Z}$ is semisimple and ${\overline{\Gamma_{\mu}}}^{Z}={\overline{\Gamma_{\mu}}}^{Z, n c}$. Then:

(i) Any $\mu$-stationary probability measure on $V$ is supported by $V^{\Gamma_{\mu}}$.

(ii) Any $\mu$-stationary probability measure on $Y$ is supported by $Y^{\Gamma_{\mu}}$.

Remark 5.18. This is not always the case when the Zariski closure of $\Gamma_{\mu}$ is only assumed to be reductive. For instance, for $V=\left(\mathbb{Q}_{p}\right)^{2}, p \neq \infty$, and $\mu=\frac{1}{2}\left(\delta_{a_{1}}+\delta_{a_{2}}\right)$ with

$$
a_{1}=\left(\begin{array}{cc}
1 & 0 \\
0 & p
\end{array}\right) \text { and } a_{2}=\left(\begin{array}{cc}
1 & p \\
p & p
\end{array}\right)
$$

the semigroup of $\mathrm{GL}\left(2, \mathbb{Q}_{p}\right)$ generated by $a_{1}$ and $a_{2}$ acts strongly irreducibly on $V$. All the vectors of the semiorbit of $(1,0)$ have norm 1 , and the closure of this semiorbit supports a $\mu$-stationary probability measure which is not $\Gamma$-invariant. 
We let $\mathbb{Q}_{\mathcal{S}}^{\times}$be the multiplicative group of $\mathbb{Q}_{\mathcal{S}}$. For $t=\left(t_{p}\right) \in \mathbb{Q}_{\mathcal{S}}$, we set $|t|=\prod_{p \in \mathcal{S}}\left|t_{p}\right|_{p}$

Proof of Lemma 5.17. (i) We may assume that $V$ is irreducible and non-trivial. We want to prove that the only $\mu$-stationary probability measure on $V$ is $\delta_{0}$. Assume $\eta$ is such a probability measure. We will again use the one-sided Bernoulli shift $(B, \mathcal{B}, \beta, T)$ whose alphabet is $(\mathrm{GL}(V), \mu)$. The probability measure $\eta$ being $\mu$ stationary, the transformation $R: B \times V \rightarrow B \times V ;(b, v) \mapsto\left(T b, b_{0} v\right)$ preserves the probability measure $\beta \otimes \eta$. Fix a norm on $V$ and set $\varphi(b, v)=\|v\|, b \in B$, $v \in V$. By [9], for any $v$ in $V \backslash\{0\}$, for $\beta$-almost any $b$ in $B$, one has $\varphi\left(R^{n}(b, v)\right)=$ $\left\|b_{n-1} \cdots b_{0} v\right\| \underset{n \rightarrow \infty}{\longrightarrow} \infty$. By Poincaré's recurrence theorem, we get $\eta=\delta_{0}$.

(ii) For any non-zero family $\mathbf{n}=\left(n_{p}\right)_{p \in \mathcal{S}}$ of non-negative integers, we set $Y_{\mathbf{n}}$ to be the set of $\lambda_{W} \in Y$ with $\operatorname{dim} W_{p}=n_{p}$, for all $p$ in $\mathcal{S}$. The space $Y$ is a finite union of such spaces $Y_{\mathbf{n}}$. We want to prove that there is no $\mu$-stationary probability measure $\eta$ on $Y_{\mathbf{n}} \backslash Y_{\mathbf{n}}^{\Gamma}$.

First case. Assume that all the $n_{p}$ are equal to 1 . In this case, we set $A \subset \mathbb{Q}_{\mathcal{S}}^{\times}$to be the subgroup $A:=\left\{t \in \mathbb{Q}_{\mathcal{S}}^{\times}|| t \mid=1\right\}$ so that the space $Y_{\mathbf{n}}$ is isomorphic to the quotient space $\left(\prod_{p \in \mathcal{S}}\left(V_{p} \backslash\{0\}\right)\right) / A$. The proof then proceeds in the same way as the one for $(i)$ by using the function

$$
\varphi(b, v)=\prod_{p \in \mathcal{S}}\left\|v_{p}\right\|
$$

which is well defined on this set of $A$-orbits.

General case. This can be reduced to the first one by replacing $V$ with the product $\prod_{p \in \mathcal{S}} \Lambda^{n_{p}} V_{p}$ and each $\lambda_{W} \in Y_{\mathbf{n}}$ with the associated Haar measure on $\prod_{p \in \mathcal{S}} \Lambda^{n_{p}} W_{p}$.

Let $G$ be an $\mathcal{S}$-adic Lie group. Recall that a probability measure $\mu$ on $G$ is said to be Ad-semisimple if the group $H_{\mu}=\overline{\mathrm{Ad}}_{\mu}$ ' is semisimple. In this case, there are strong restrictions on the homogeneous spaces of $G$ which carry a $\mu$-stationary probability measure.

Proposition 5.19 (Stationary measures in $G / G^{\prime}$ ). Let $G$ be a weakly regular $\mathcal{S}$-adic Lie group and $\mu$ be an Ad-semisimple probability measure on $G$ such that $\left(G, \Gamma_{\mu}\right)$ has good exponentials, $H_{\mu}=H_{\mu}^{n c}$ and $\int_{G} \log \|\operatorname{Ad} g\| \mathrm{d} \mu(g)<\infty$.

Let $G^{\prime} \subset G$ be a unimodular closed subgroup. Assume there exists a $\mu$-ergodic $\mu$-stationary probability measure $\eta$ on the homogeneous space $G / G^{\prime}$. Then, there exists $x=g G^{\prime}$ in $G / G^{\prime}$ and a semiconnected component $S$ of $g G^{\prime} g^{-1}$ such that the unimodular normalizer $N_{1}(S)$ contains $\Gamma_{\mu}$ and $\eta\left(N_{1}(S) x\right)=1$.

By definition, the unimodular normalizer $N_{1}(S)$ of the unimodular subgroup $S$ is the subgroup of the normalizer of $S$ whose elements preserve the Haar measure of $S$. It contains $g G^{\prime} g^{-1}$.

When $G$ is a real Lie group, the conclusion of Proposition 5.19 implies that the connected component of $g G^{\prime} g^{-1}$ is normalized by $\Gamma_{\mu}$.

Proof of Proposition 5.19 when $G$ is a real Lie group. We will use Lemma 5.17 with $V=\mathfrak{g}$ and $Y$ the set of Haar measures $\lambda_{W}$ on sub- $\mathbb{Q}_{\mathcal{S}}$-modules $W \subset \mathfrak{g}$. Since $G^{\prime}$ is unimodular, any Haar measure $y=\lambda_{\mathfrak{g}^{\prime}} \in Y$ is a $G^{\prime}$-invariant element of $Y$. The orbit $G y \subset Y$ then supports a $\mu$-stationary probability measure: this 
is the image $\eta^{\prime}$ of $\eta$ by the natural map $G / G^{\prime} \rightarrow G y$. We may assume that $y$ is in the support of $\eta^{\prime}$. By Lemma 5.17, this $\mu$-stationary probability measure $\eta^{\prime}$ is supported by the set of $\Gamma_{\mu}$-invariant vectors. In particular, $y$ is $\Gamma_{\mu}$-invariant. Since $\eta^{\prime}$ is also $\mu$-ergodic, it is a Dirac mass: $\eta^{\prime}=\delta_{y}$. This implies that $\Gamma_{\mu}$ is included in the unimodular normalizer $N_{1}\left(\mathfrak{g}^{\prime}\right)$ of $\mathfrak{g}^{\prime}$ and that the probability measure $\eta$ is supported by an orbit of $N_{1}\left(\mathfrak{g}^{\prime}\right)$.

This ends the proof when $G$ is a real Lie group, since in this case $N_{1}\left(\mathfrak{g}^{\prime}\right)$ is also the unimodular normalizer of the connected component $S$ of $G^{\prime}$.

\subsection{Stationary measures for $\mathcal{S}$-adic groups.}

The reader who is only interested in real Lie groups may skip this section.

The strategy for $\mathcal{S}$-adic Lie groups is the same as the one for real Lie groups. The additional difficulty comes from the existence of the following example which tells us that we will once more have to use the existence of $\eta$ to conclude.

Example 5.20. The normalizer of the Lie algebra $\mathfrak{g}^{\prime}$ of a subgroup $G^{\prime} \subset G$ does not always normalize a semiconnected component of $G^{\prime}$.

An example is $G=\operatorname{SL}\left(d, \mathbb{Q}_{p}\right)$ and $G^{\prime}=\operatorname{SL}\left(d, \mathbb{Z}_{p}\right)$, for $p<\infty$.

Another example is $G=\operatorname{SL}\left(d, \mathbb{Q}_{p}\right) \ltimes \mathbb{Q}_{p}^{2}$ and $G^{\prime}=\mathbb{Q}_{p} \times \mathbb{Z}_{p} \subset \mathbb{Q}_{p}^{2}$ for which the normalizer of a semiconnected component of $G^{\prime}$ is not even open in the normalizer of $\mathfrak{g}^{\prime}$.

We shall need the following.

Lemma 5.21. Let $G$ be an $\mathcal{S}$-adic Lie group that is a closed subgroup of a product, for $p$ in $\mathcal{S}$, of p-adic Lie groups. Assume $G^{\prime}$ is a closed normal subgroup of $G$ and the adjoint action of $G$ on $G^{\prime}$ is unimodular. Then, so is the adjoint action of $G$ on $G_{u}^{\prime}$.

Proof. Let $\mathfrak{g}^{\prime}$ be the Lie algebra of $G^{\prime}$ and $\mathfrak{g}_{u}^{\prime}$ be the one of $G_{u}^{\prime}$. By Lemma 5.4, for any $g$ in $G$, the sum of the generalized eigenspaces of $\mathrm{Ad} g$ which are associated to eigenvalues with modulus $\neq 1$ is contained in $\mathfrak{g}_{u}^{\prime}$. Hence, we get $\left|\operatorname{det}^{\prime} \mathfrak{g}^{\prime}(\operatorname{Ad} g)\right|=$ $\left|\operatorname{det}_{\mathfrak{g}_{u}^{\prime}}(\operatorname{Ad} g)\right|$. The result follows.

Proof of Proposition 5.19 in the general case. We may assume that the base point $x_{0}$ of $X:=G / G^{\prime}$ belongs to the support of $\eta$. Reasoning as in the real case we get $\eta\left(N_{1}\left(\mathfrak{g}^{\prime}\right) x_{0}\right)=1$ and $\Gamma_{\mu} \subset N_{1}\left(\mathfrak{g}^{\prime}\right)$, where $\mathfrak{g}^{\prime}$ is the Lie algebra of $G^{\prime}$, so that we can assume from now on that $N_{1}\left(\mathfrak{g}^{\prime}\right)=G$. In the same way, as, by Lemma [5.21, the action of $G^{\prime}$ on $\mathfrak{g}_{u}^{\prime}$ is unimodular, we can also assume $N_{1}\left(\mathfrak{g}_{u}^{\prime}\right)=G$ and hence, $G$ being weakly regular, $N_{1}\left(G_{u}^{\prime}\right)=G$. Let $\mathfrak{v}$ be the sum of the $\Gamma_{\mu}$-irreducible sumodules of $\mathfrak{g}^{\prime}$ on which $\operatorname{Ad} \Gamma_{\mu}$ is unbounded. We want to construct the group $S$ by applying the same strategy as the one used in the proof of Lemma 5.6. We will prove that $\mathfrak{v} \subset \mathfrak{g}_{u}^{\prime}$.

Since $\left(G, \Gamma_{\mu}\right)$ has good exponentials and $\mu$ has a finite first moment, we can apply Proposition 5.13 to $\mathfrak{g}^{\prime}$ and the probability measure $\check{\mu}$ on $G$. For $\beta$-almost every $b$ in $B$, we get a subalgebra $\mathfrak{v}_{\breve{b}}$ of $\mathfrak{g}^{\prime}$ and a subgroup $V_{\breve{b}}=\exp _{N}\left(\mathfrak{v}_{\breve{b}}\right)$ of $G$ such that, for $\beta$-almost any $b$ in $B$, the norm of the restriction of $\operatorname{Ad}\left(b_{n-1} \cdots b_{0}\right)$ to $\mathfrak{v}_{\breve{b}}$ goes to 0 as $n \rightarrow \infty$. We claim that, for $\beta$-almost any $b$ in $B, V_{\breve{b}}$ is contained in $G^{\prime}$. Indeed, equip the space $B \times G / G^{\prime}$ with the map $R:(b, x) \mapsto\left(T b, b_{0} x\right)$ which 
preserves the probability measure $\beta \otimes \eta$. For $\beta \otimes \eta$-almost any $(b, x)$ in $B \times G / G^{\prime}$, set

$$
\varphi(b, x)=\inf \left\{\|v\| \| v \in \mathfrak{v}_{\check{b}} \exp _{N}(v) x \neq x\right\} \in[0, \infty] .
$$

As $\mathfrak{v}_{\breve{b}} \subset \mathfrak{g}^{\prime}$, one has $\varphi(b, x)>0$, and we shall prove that $\varphi=\infty$ almost everywhere. But, for $\beta \otimes \eta$-almost every $(b, x)$, one has, for any $n, \varphi\left(R^{n}(b, x)\right) \leq$ $\left\|b_{n-1} \cdots b_{0} \mid \mathfrak{v}_{\dot{b}}\right\| \varphi(b, x)$, and therefore, if $\varphi(b, x)<\infty, \varphi\left(R^{n}(b, x)\right) \underset{n \rightarrow \infty}{\longrightarrow} 0$, which contradicts Poincaré's recurrence theorem. Thus, $\varphi=\infty$ and, for $\beta \otimes \eta$-almost any $(b, x), V_{\breve{b}} x=x$. In other words, for $\beta$-almost any $b$ in $B$, we have $\mathfrak{v}_{\breve{b}} \subset \mathfrak{g}_{u}^{\prime}$. In particular, as, for any Borel subset $E$ of $B$ with $\beta(E)=1$, the set $\bigcup_{b \in E} \mathfrak{v}_{\breve{b}}$ spans $\mathfrak{v}$, we get $\mathfrak{v} \subset \mathfrak{g}_{u}^{\prime}$.

We can now finish the construction of the group $S$. Let $\mathfrak{l}^{\prime}$ be the space of $\Gamma_{\mu^{-}}$ invariant vectors of $\mathfrak{g}^{\prime}$. Since $H_{\mu}=H_{\mu}^{n c}$, one has $\mathfrak{g}^{\prime}=\mathfrak{l}^{\prime} \oplus \mathfrak{v}$. Let $\Omega$ be a standard open subset of $G$ with $\left(G, \Gamma_{\mu}\right)$-good exponential map $\exp _{\Omega}: O \rightarrow \Omega$. Then, $\Gamma_{\mu}$ centralizes $\exp _{\Omega}\left(O \cap \mathfrak{l}^{\prime}\right)$. Assume $\Omega$ is small enough to have $\exp _{\Omega}\left(O \cap \mathfrak{g}^{\prime}\right) \subset \exp _{\Omega}(O \cap$ $\left.\mathfrak{l}^{\prime}\right) G_{u}^{\prime}$ so that $\Gamma_{\mu}$ normalizes $\exp _{\Omega}\left(O \cap \mathfrak{g}^{\prime}\right) G_{u}^{\prime}$. We set $S=\exp _{\Omega}\left(O \cap \mathfrak{g}^{\prime}\right) G_{\infty}^{\prime} G_{u}^{\prime}$. As $G_{\infty}^{\prime}$ and $G_{u}^{\prime}$ are normal subgroups of $G$ and $\Omega$ normalizes $\exp _{\Omega}\left(O \cap \mathfrak{g}^{\prime}\right), S$ is normalized by $\Omega$. Hence the normalizer $N_{1}(S)$ of $S$ is open in $G$. In particular, for $\eta$-almost any $x$ in $X$, we have $\eta\left(N_{1}(S) x\right)>0$ and, as $\Gamma_{\mu}$ normalizes unimodularly $S$, this orbit is $\Gamma_{\mu}$-invariant. As $\eta$ is ergodic, we get $\eta\left(N_{1}(S) x_{0}\right)=1$, which should be proved.

\subsection{Convergence of cocompact lattices.}

Here we prove a general fact on the restriction of the Chabauty topology to the set of cocompact lattices. We will use this fact in section 5.9 .

The following proposition tells us that for cocompact lattices "algebraic convergence" coincides with "geometric convergence".

Proposition 5.22 (Chabauty topology for cocompact lattices). Let $G$ be a compactly generated locally compact group and $\Lambda_{\infty}$ be a cocompact lattice in $G$. Then the group $\Lambda_{\infty}$ is finitely generated.

Let $\left(\Lambda_{n}\right)$ be a sequence of lattices in $G$ which converges to $\Lambda_{\infty}$ in the Chabauty topology. Then, there exists $n_{0}$ and a compact subset $C$ of $G$ such that, for $n \geq n_{0}$, $\Lambda_{n}$ is cocompact in $G$ and $G=C \Lambda_{n}$.

Remark 5.23. When $G$ is not compactly generated such a compact "upper bound" $C$ for suitable fundamental domains of $\Lambda_{n}$ may not exist, even if $\Lambda_{n}$ avoids a fixed neighborhood of $e$ in $G$ and the covolume of $\Lambda_{n}$ converges to the covolume of $\Lambda$.

Proof of Proposition 5.22. Let $B$ be a symmetric relatively compact open subset generating $G$ and containing the identity element of $G$ so that we have $G=$ $\bigcup_{k \geq 1} B^{k}$. We may assume that $B$ contains a fundamental domain for $\Lambda_{\infty}$, i.e. that $G=B \Lambda_{\infty}$.

As $\overline{B^{2}}$ is compact, there exists a finite subset $F_{\infty}$ of $\Lambda_{\infty}$ such that

$$
\overline{B^{2}} \subset B F_{\infty} .
$$

By induction on $k \geq 1$, one gets the inclusions

$$
B^{k} \subset B\left(F_{\infty}\right)^{k-1} .
$$


Indeed, one has $B^{k+1}=B B^{k} \subset B B\left(F_{\infty}\right)^{k-1} \subset B\left(F_{\infty}\right)^{k}$. Let $\Lambda_{\infty}^{\prime}$ be the subgroup of $\Lambda_{\infty}$ generated by $F_{\infty}$. One gets $G=B \Lambda_{\infty}^{\prime}$, and $\Lambda_{\infty}^{\prime}$ is thus a cocompact lattice in $G$. Hence $\Lambda_{\infty}^{\prime}$ has finite index in $\Lambda_{\infty}$ and $\Lambda_{\infty}$ is finitely generated.

Since the lattice $\Lambda_{n}$ converges to $\Lambda_{\infty}$ in the Chabauty topology, there exist finite subsets $F_{n} \subset \Lambda_{n}$ which converge to $F_{\infty}$. Using inclusion (5.7) in which $\overline{B^{2}}$ is compact and $B$ is open, we deduce that, for $n$ large enough, one has

$$
\overline{B^{2}} \subset B F_{n} \text {. }
$$

The same induction argument gives, for all $k \geq 1$,

$$
B^{k} \subset B\left(F_{n}\right)^{k-1}
$$

hence, $G=B \Lambda_{n}$. The result follows.

\subsection{Lattices in $\mathcal{S}$-adic Lie groups.}

The reader who is only interested in real Lie groups may skip this section. In Corollary 5.24 we show how to extend Proposition 5.22 to $\mathcal{S}$-adic Lie groups. We also prove a version of the Auslander Theorem for lattices in $\mathcal{S}$-adic Lie groups. We will use these facts in section 5.9 .

A weakly regular semiconnected $\mathcal{S}$-adic Lie group may fail to be compactly generated, as for instance $G=\mathbb{R} \times \mathbb{Q}_{p}$, which contains the lattice $\Lambda=\mathbb{Z}\left[\frac{1}{p}\right]$. Even though the lattices in $G$ are not finitely generated, the conclusion of Proposition 5.22 is still true for these groups.

Corollary 5.24. Let $G$ be a weakly regular semiconnected $\mathcal{S}$-adic Lie group such that $G_{u}$ is nilpotent, and let $\Lambda_{\infty}$ be a lattice in $G$ and $\left(\Lambda_{n}\right)$ be a sequence of lattices in $G$ which converges to $\Lambda_{\infty}$ in the Chabauty topology. Then there exists a compact subset $C \subset G$ such that, for all $n \geq 1, G=C \Lambda_{n}$.

Note that, by [8, Prop. 5.1], lattices in $G$ are necessarily cocompact. To get Corollary 5.24 from Proposition 5.22 , we will need to use some facts on the structure of these groups $G$.

Lemma 5.25. Let $Z$ be an abelian $\mathcal{S}$-adic Lie group which is isomorphic to a product of vector spaces over the fields $\mathbb{Q}_{p}, p \in \mathcal{S}$. Let $K$ be a compact abelian group. If there exists a morphism $Z \rightarrow K$ with dense image, then $K$ is connected.

Proof. As any non-connected group admits proper open subgroups, it suffices to prove that, if $K$ is finite, $K$ is trivial. But in this case the morphism $Z \rightarrow K$ is onto and its kernel is a finite index subgroup of $Z$. By assumption, such a subgroup equals $Z$, which should be proved.

We can now begin the study of cocompact groups in nilpotent semiconnected groups.

Lemma 5.26. Let $G$ be a nilpotent weakly regular $\mathcal{S}$-adic Lie group with $G=G_{u}$ and $G_{\infty}=\{e\}$. Then $G$ admits no proper cocompact closed subgroups.

Proof. We argue by induction on the dimension of $G$. If it is zero, there is nothing to prove. If not, we consider the center $Z$ of $G$. By [26, Prop. 2.1], it is isomorphic to a product of vector spaces over $\mathbb{Q}_{p}, p \in \mathcal{S}$. Let $H$ be a closed cocompact subgroup of $G$ and set $L=\overline{Z H}$. As $H$ is contained in $L, L / H$ is compact. As $[L, L] \subset H$, $H$ is normal in $L$. Now, the morphism $Z \rightarrow L / H$ has dense image so that, by 
Lemma 5.25 $L / H$ is connected. As $G_{\infty}=\{e\}, L / H$ is trivial; that is, $Z \subset H$. Now, we may view $H$ as a closed cocompact subgroup of $G / Z$ which, by [26. Prop. 2.1 , is still a weakly regular group. By induction, we get $H=G$, which should be proved.

From this, we deduce

Lemma 5.27. Let $G$ be a weakly regular semiconnected $\mathcal{S}$-adic Lie group such that $G_{u}$ is nilpotent and let $H \subset G$ be a closed cocompact subgroup containing $G_{\infty}$. Then $H$ contains $G_{u}$.

Proof. Let $\Omega$ be a standard open subset of $G$. We assume $G$ is $\Omega$-semiconnected. We have $G=\Omega_{f} G_{u, f} G_{\infty}$. By [26, Prop. 2.1 and Th. 2.1], we may write $G_{u, f}$ as the union of an increasing sequence of compact subgroups $\left(K_{n}\right)$. As $\Omega_{f}$ is compact, we may suppose, for any $n, \Omega_{f}$ normalizes $K_{n}$.

We may view $H$ as a closed cocompact subgroup of $\Omega_{f} G_{u, f}$. We claim that the group $L=H G_{u, f}$ is closed. Indeed, for any $n$, the group $\left(H \cap\left(\Omega_{f} K_{n}\right)\right) K_{n}$ is closed since it is compact and we have $H G_{u, f}=\bigcup_{n}\left(H \cap\left(\Omega_{f} K_{n}\right)\right) K_{n}$. Now, $\Omega_{f} G_{u, f}$ having no real factor, by [26, Cor 1.1], the union of an increasing sequence of closed subgroups of $\Omega_{f} G_{u, f}$ is still closed. Hence $L=H G_{u, f}$ is closed.

In particular, $H \cap G_{u, f}$ is a cocompact subgroup of $G_{u, f}$. By Lemma 5.26, we have $G_{u, f} \subset H$, which should be proved.

This gives

Lemma 5.28. Let $G$ be a weakly regular semiconnected $\mathcal{S}$-adic Lie group such that $G_{u}$ is nilpotent. There exists a compactly generated open subgroup $G^{\prime}$ of $G$ such that, for any cocompact closed subgroup $H$ of $G$, one has $G=G^{\prime} H$.

Proof. Let $G^{\prime}$ be an open compactly generated subgroup of $G$ which maps onto the compact group $G / G_{u} G_{\infty}$ and let $H$ be a cocompact closed subgroup of $G$. By Lemma 5.27, we have $G_{u} \subset \overline{G_{\infty} H}$; hence, $G^{\prime}$ being open and containing $G_{\infty}$, $G_{u} \subset G^{\prime} H$. We get $G=G^{\prime} G_{u} \subset G^{\prime} H$, which should be proved.

Proof of Corollary [5.24. Let $G^{\prime}$ be as in Lemma 5.28. The intersections $\Lambda_{n}^{\prime}:=$ $\Lambda_{n} \cap G^{\prime}$ and $\Lambda_{\infty}^{\prime}=\Lambda_{\infty} \cap G^{\prime}$ are lattices in $G^{\prime}$. By [8, Prop. 5.1], these lattices are cocompact. By Proposition 5.22, there exists a compact subset $C$ of $G^{\prime}$ such that, for all $n \geq 1$, one has $G^{\prime}=C \Lambda_{n}^{\prime}$. But then, one also has $G=C \Lambda_{n}$.

We end this section with a few structure results on semiconnected groups and their lattices that we will need in the course of the proof of Proposition 5.32 . We recall that the amenable radical of a locally compact group is its largest amenable closed normal subgroup.

Lemma 5.29 (Structure of the amenable radical). Let $G$ be a weakly regular semiconnected $\mathcal{S}$-adic Lie group with Lie algebra $\mathfrak{g}$. Let $\mathfrak{r}$ be the smallest ideal of $\mathfrak{g}$ containing the solvable radical $\mathfrak{r}_{0}$ of $\mathfrak{g}$ such that $\mathfrak{g}=\mathfrak{r}+\mathfrak{g}_{u}$, let $\mathfrak{l}:=\mathfrak{g} / \mathfrak{r}$ and $R$ be the kernel of the adjoint map $G \rightarrow$ Aut $(\mathfrak{l})$. Then $R$ is the amenable radical of $G$. If $\Omega$ is a standard open subset of $G$ such that $G$ is $\Omega$-semiconnected and $R_{\Omega}$ is the $\Omega$-semiconnected component of $R$, then $R_{\Omega}$ is normal in $G$ and $R / R_{\Omega}$ centralizes an open finite index subgroup of $G / R_{\Omega}$. 
Proof. By [26, Sect. 2] one has $\mathfrak{r}_{u}=\mathfrak{r} \cap \mathfrak{g}_{u}$. By the definition of $R$, for any $r$ in $R$ and $X$ in $\mathfrak{g}_{u}$, one has $\operatorname{Ad} r(X)-X \in \mathfrak{r}_{u}$; hence for any $g$ in $G_{u}$, one has $\mathrm{rgr}^{-1} \in g R_{u}$. Therefore $\left[G_{u}, R\right] \subset R_{u} \subset R_{\Omega}$ and $G_{u}$ normalizes $R_{\Omega}$. Let $\exp _{\Omega}: O \rightarrow \Omega$ be the exponential map of $\Omega$. We have $R \cap \Omega=\exp _{\Omega}(O \cap \mathfrak{r})$ so that $\Omega$ normalizes $R \cap \Omega$ and hence $R_{\Omega}$. As $G=\Omega G_{u} G_{\infty}$ and, by standard real Lie groups theory, $G_{\infty} \subset G_{u} R_{\infty}, G$ normalizes $R_{\Omega}$. Besides, $G_{1}=G_{u} R_{\Omega}$ is a finite index open subgroup of $G$ and $\left[G_{1}, R\right] \subset R_{\Omega}$; that is, $R / R_{\Omega}$ centralizes $G_{1} / R_{\Omega}$. In particular, setting $R_{1}:=R \cap G_{1}$, we get $\left[R_{1}, R_{1}\right] \subset R_{\Omega}$ and, as $R_{\Omega}$ is a compact extension of a solvable group, $R_{1}$ is amenable. Since $R_{1}$ has finite index in $R, R$ is amenable. Now, $G / R$ is isomorphic to an open finite index subgroup of $\operatorname{Aut}(\mathfrak{l})$. As the group $\operatorname{Aut}(\mathfrak{l})$ is semisimple with no anisotropic factor, the amenable radical of $G / R$ is trivial. Hence $R$ is the amenable radical of $G$.

For semiconnected groups, we have the following analogue of Auslander's Projection Theorem.

Lemma 5.30 (Projection of lattices). Let $G$ be a weakly regular semiconnected $\mathcal{S}$-adic Lie group with amenable radical $R$ and let $\Lambda$ be a lattice in $G$. Then $\Lambda R / R$ is a lattice in $G / R$ and $R \cap \Lambda$ is a lattice in $R$.

Proof. The proof follows along the same lines as the one of Auslander's Theorem in the real case (see [24, Th. 8.24]) by using the structure results of [26, Sect. 2] and Lemma 5.29. We fix a standard open subset $\Omega$ of $G$ such that $G$ is $\Omega$-semiconnected. By Lemma [5.29, we can assume $[G, R] \subset R_{\Omega}$. We let $\mathfrak{r}$ be the Lie algebra of $R$, $\mathfrak{r}_{0} \subset \mathfrak{r}$ be the solvable radical of $\mathfrak{g}$ and $N_{u}$ be the subgroup of $G$ spanned by the Ad-unipotent one-parameter subgroups of $G$ whose derivative belongs to $\mathfrak{r}_{0}$. By 26. Prop. 2.1 and Th. 2.1], the group $N_{u}$ is isomorphic to a product of algebraic unipotent groups over $\mathbb{Q}_{p}, p \in \mathcal{S}$. Let $R_{0, \infty}$ be the radical of $G_{\infty}$, that is, the closed connected subgroup of $G_{\infty}$ with Lie algebra $\mathfrak{r}_{0, \infty}$, and set $R_{2}:=N_{u} R_{0, \infty}$. Let $F$ be the closure of the group $\Lambda R_{2}$ in $G$. Reasoning as in the proof of [24, Th. 8.24], one proves that the Lie algebra $\mathfrak{f}$ of $F$ is solvable. As $R_{\Omega}$ is a compact extension of $R_{2}$ and, by Lemma 5.29. $R_{\Omega}$ is a normal subgroup of $G, F R_{\Omega}$ is a closed subgroup of $G$ which is the closure of $\Lambda R_{\Omega}$. By Borel's density theorem and Lemma 5.29, the Lie algebra $(\mathfrak{f}+\mathfrak{r}) / \mathfrak{r}=\mathfrak{f} /(\mathfrak{f} \cap \mathfrak{r})$ is a solvable ideal of the semisimple Lie algebra $\mathfrak{g} / \mathfrak{r}$. Therefore it is trivial and $\mathfrak{f}$ is contained in $\mathfrak{r}$; that is, $R_{\Omega}$ is open, hence closed, in $F R_{\Omega}$ and $F R_{\Omega}=\Lambda R_{\Omega}$. In other words, the image of $\Lambda$ in $G / R_{\Omega}$ is discrete and $\Lambda \cap R_{\Omega}$ is a lattice in $R_{\Omega}$. Now the group $G / R_{\Omega}$ is a central extension of $G / R$, which is isomorphic to an open finite index subgroup of $\operatorname{Aut}(\mathfrak{g} / \mathfrak{r})$. Thus, again reasoning as in the proof [24, Th. 8.24], we get that $\Lambda \cap R$ is a lattice in $R$.

\subsection{Quotient space and injectivity radius.}

The following proposition compares the injectivity radius in a quotient $\underline{X}=X / S$ of $X$ with the injectivity radius in $X$. It will be used in the proof of Proposition 7.8 .

Fix a standard open subset $\Omega$ of $G$ and $r_{0}>0$ such that $B\left(0, r_{0}\right) \subset O=\log \Omega$. We recall from (2.1) that, for every $x$ in $X$, the injectivity radius $r_{X}(x)$ of $X$ at $x$ is the upper bound of the radii $r \leq r_{0}$ for which the map $\mathfrak{g} \mapsto X ; v \mapsto \exp _{\Omega}(v) x$ is injective on the ball $B(0, r) \subset \mathfrak{g}$. Let $S$ be a closed normal subgroup of $G$ such that $S \cap \Lambda$ is a lattice in $S$. Then, we set $X \rightarrow \underline{X}:=X / S=G / S \Lambda$ and define the injectivity radius $r_{\underline{X}}$ by using the Lie algebra $\mathfrak{g} / \mathfrak{s}$ of $G / S$. 
Definition 5.31. We will say that $r_{X}$ controls $r_{X}$ if $r_{X}$ is bounded below when $r_{X}$ is bounded below, i.e., with quantifiers,

$$
\forall \varepsilon>0 \quad \exists \alpha>0 \quad \forall x \in X \quad\left(r_{X}(x) \geq \varepsilon \Longrightarrow r_{\underline{X}}(\underline{x}) \geq \alpha\right) .
$$

Proposition 5.32 (Control of the injectivity radius). Let $G$ be a weakly regular $\mathcal{S}$-adic Lie group, $\Lambda$ be a discrete subgroup of $G, X=G / \Lambda$ and $S \subset G$ be a semiconnected closed normal subgroup of $G$ such that $S \cap \Lambda$ is a lattice in $S$ and the adjoint action of $G$ on $S$ is unimodular. Let $\underline{X}:=G / S \Lambda$. Then the injectivity radius of $X$ controls the injectivity radius of $\underline{X}$.

Remark 5.33. It is important to assume that the action of $G$ on $S$ by conjugation is volume preserving. For example, if $G$ is the real Lie group $G:=\mathbb{R}^{2} \rtimes \mathbb{R}^{\times}$where the adjoint action of $t \in \mathbb{R}^{\times}$on $\mathbb{R}^{2}$ is given by the diagonal matrix $\operatorname{diag}\left(t, t^{-1}\right)$, if $\Lambda$ is the discrete subgroup $\Lambda:=\mathbb{Z}^{2}$ and if $S$ is the normal subgroup $S:=\mathbb{R} \times\{0\}$, one has $\inf _{X} r_{X}>0$ while $\inf _{\underline{X}} r_{\underline{X}}=0$.

The following corollary is an immediate consequence of Proposition 5.32 and of the definition of exponential $\mu$-unstability of the cusps (see Section 2.3).

Corollary 5.34. Let $G, \Lambda, S, X, \underline{X}$ be as in Proposition 5.32. Let $\mu$ be a probability measure on $G$. If the cusps of $X$ are exponentially $\mu$-unstable, then so are the cusps of $\underline{X}$.

In order to prove Proposition [5.32, one must first control the injectivity radius in the quotient of $X$ by the amenable radical $R$ of $S$.

Lemma 5.35. Let $G$ be a weakly regular $\mathcal{S}$-adic Lie group, $\Lambda$ be a discrete subgroup of $G$ and $S$ be a semiconnected normal subgroup of $G$ such that $S \cap \Lambda$ is a lattice in $S$ and the adjoint action of $G$ on $S$ is unimodular. Let $R$ be the amenable radical of $S$. Then the injectivity radius of $G / \Lambda$ controls the injectivity radius of $G / R \Lambda$.

Proof. By Lemma 5.30, $\Lambda \cap R$ is a lattice in $R$. Let $\Omega$ be a standard open subset of $G$ such that $S$ is $\Omega$-semiconnected and let $R_{\Omega}$ be the $\Omega$-semiconnected component of $R$. By Lemma $5.29, R_{\Omega}$ is normal in $S$.

Assume by contradiction that there exists a sequence $x_{n}=g_{n} \Lambda$ in $X$ and $\varepsilon_{0}>0$ such that, setting $x_{n}^{\prime}:=g_{n} R \Lambda$ for the images in $X^{\prime}:=G / R \Lambda$,

$$
\inf _{n \geq 1} r_{X}\left(x_{n}\right) \geq \varepsilon_{0} \text { and } r_{X^{\prime}}\left(x_{n}^{\prime}\right) \underset{n \rightarrow \infty}{\longrightarrow} 0 .
$$

By definition, $r_{X}\left(x_{n}\right)$ is bounded by the systole of the lattice $\Lambda_{n}:=g_{n} \Lambda g_{n}^{-1}$, i.e. one has

$$
r_{X}\left(x_{n}\right) \leq \inf \left\{\|v\| \leq r_{0} \mid v \in \mathfrak{g} \backslash\{0\}, \quad e^{v} \in \Lambda_{n}\right\} .
$$

We set $\Delta=\Lambda \cap R, \Delta_{n}=\Lambda_{n} \cap R=g_{n} \Delta g_{n}^{-1}$ and $\Gamma_{n}=\Delta_{n} \cap R_{\Omega}$. Since the actions of $G$ by conjugation on $\mathfrak{s}$ and the one of $\operatorname{Aut}(\mathfrak{s} / \mathfrak{r})$ on $\mathfrak{s} / \mathfrak{r}$ are unimodular, so is the action of $G$ on $R$, and the lattices $\Delta_{n}, n \geq 1$, all have the same covolume in $R$. As $R_{\Omega}$ is an open subgroup of $R$, for any $n, \Gamma_{n}$ is a lattice in $R_{\Omega}$.

Now, the first condition in (5.8) implies that these lattices $\Delta_{n}$ avoid a fixed neighborhood of $e$ in $R$. According to Mahler-Chabauty's compactness theorem (see [13]), after eventually extracting a subsequence, we can assume the sequence of lattices $\Delta_{n}$ converges in Chabauty's topology towards a lattice $\Delta_{\infty}$ of $R$. As $R_{\Omega}$ is open in $R, \Gamma_{n}$ converges to the lattice $\Gamma_{\infty}=\Delta_{\infty} \cap R_{\Omega}$ of $R_{\Omega}$. 
By Corollary 5.24 and Lemma 5.29, there exists a compact subset $C$ of $R_{\Omega}$ such that

$$
R_{\Omega}=C \Gamma_{n} \quad \text { for all } n \geq 1 .
$$

Since the number of disjoint $\varepsilon$-balls in $C$ is uniformly bounded, looking at representatives in $C$ modulo $\Gamma_{n}$ of powers $r^{N}$ of an element $r \in R_{\Omega}$, one deduces the folowing fact.

For any $\varepsilon>0$, there exists $N \geq 1$ such that for all $r \in R_{\Omega}$, one can find distinct integers $N_{1}, N_{2}$ bounded by $N$, and $v \in \mathfrak{r}$ with $\|v\| \leq \varepsilon$ such that

$$
r^{N_{1}} \in e^{v} r^{N_{2}} \Delta_{n} .
$$

The second condition in (5.8) tells us that there exists a sequence

$$
\lambda_{n}=e^{w_{n}} r_{n} \in \Lambda_{n}, \text { with } r_{n} \in R, w_{n} \in \mathfrak{g} \backslash \mathfrak{r} \text { and } \lim _{n \rightarrow \infty} w_{n}=0 .
$$

Since the lattices $\Delta_{n}$ have the same covolume in $R$, the subgroups $\Delta_{n} / \Gamma_{n}=$ $\Delta_{n} R_{\Omega} / R_{\Omega}, n \geq 1$, of the discrete group $R / R_{\Omega}$ have a uniformly bounded index. Hence, after raising (5.11) to a fixed power, we can assume, for any $n, r_{n} \in R_{\Omega} \Delta_{n}$. Using (5.10) with $\varepsilon=\varepsilon_{0} / 4$ and $r=r_{n}$, and after again eventually extracting a subsequence, we can suppose there exist distinct integers $N_{1}, N_{2}$, such that, for every $n \geq 1$, one can write

$$
r_{n}^{N_{1}} \in e^{v_{n}} r_{n}^{N_{2}} \Delta_{n} \text { with } v_{n} \in \mathfrak{r},\left\|v_{n}\right\| \leq \varepsilon_{0} / 4 .
$$

Hence, using (5.11), for $n$ large enough, one has

$$
\lambda_{n}^{N_{1}} \in e^{v_{n}^{\prime}} \lambda_{n}^{N_{2}} \Delta_{n} \quad \text { with } \quad v_{n}^{\prime} \in \mathfrak{g},\left\|v_{n}^{\prime}\right\| \leq \varepsilon_{0} / 2 .
$$

In particular, the element $e^{v_{n}^{\prime}}$ belongs to $\Lambda_{n}$, hence according to (5.8) and (5.9), one has $v_{n}^{\prime}=0$. That is,

$$
\lambda_{n}^{N_{1}-N_{2}} \in \Delta_{n}
$$

Since $R$ is normal in $G$, this tells us that, for $n$ large, one has

$$
e^{\left(N_{1}-N_{2}\right) w_{n}} \in R
$$

Hence $w_{n}=0$ for $n$ large enough, which is a contradiction.

To deal with the general case of Proposition 5.32 we will study the quotient $G / R$.

Lemma 5.36. Let $G$ be an $\mathcal{S}$-adic Lie group and $L$ be a closed normal subgroup of $G$. Assume the Lie algebra $\mathfrak{l}$ of $L$ is semisimple with no anisotropic factor and the adjoint map $L \rightarrow \operatorname{Aut}(\mathfrak{l})$ is an isomorphism onto a finite index subgroup. Let $C$ be the centralizer of $L$ in $G$ so that $C L$ has finite index in $G$. Then, if $\Lambda$ is a discrete subgroup of $G$ such that $L \cap \Lambda$ is a lattice in $L$, the subgroup $(C \cap \Lambda)(L \cap \Lambda)$ has finite index in $\Lambda$.

Proof. Consider the adjoint map $\varphi: G \rightarrow \operatorname{Aut}(\mathfrak{l})$. As $\varphi(\Lambda)$ normalizes $\varphi(\Lambda \cap L)$, by [8, Cor. 4.8] (which directly follows from Borel's density theorem) $\varphi(\Lambda \cap L)$ has finite index in $\varphi(\Lambda)$, which should be proved. 
We can now give the

Proof of Proposition 5.32. In this proof, we may freely replace $G$ or $\Lambda$ by a finite index subgroup. In particular, let as above $R$ be the amenable radical of $S$. We can assume $G=C S$, where $C$ is the group of elements $g$ of $G$ whose image in $G / R$ commutes with $S / R$. As, by Lemma [5.29 and [26, Cor. 2.1], the image of $S$ in $G / R$ satisfies the assumptions of Lemma [5.36, we can also assume that $\Lambda=(\Lambda \cap C)(\Lambda \cap S)$.

Now, by Lemma 5.30, $R \cap \Lambda$ is a lattice in $R$ and the quotient space $X_{1}=G / R \Lambda$ identifies with $C / R(C \cap \Lambda) \times S / R(S \cap \Lambda)$. Set $X_{2}=C / R(C \cap \Lambda)=G / S \Lambda$ and $X_{3}=S / R(S \cap \Lambda)=G / C \Lambda$. For any $x_{1}=\left(x_{2}, x_{3}\right)$ in $X_{1}$, one has

$$
r_{X_{1}}\left(x_{1}\right) \leq \min \left(r_{X_{2}}\left(x_{2}\right), r_{X_{3}}\left(x_{3}\right)\right) .
$$

By Lemma 5.35, for any $\varepsilon>0$, there exists $\alpha>0$ such that, if $x$ is in $X=G / \Lambda$ and $r_{X}(x) \geq \varepsilon$, then if $x_{1}$ is the image of $x$ in $X_{1}$, one has $r_{X_{1}}\left(x_{1}\right) \geq \alpha$. Thus, if $x_{2}$ is the image of $x$ in $G / S \Lambda$, by (5.12), we get $r_{X_{2}}\left(x_{2}\right) \geq \alpha$, which should be proved.

\section{The First Return Markov CHAin}

The results of this chapter are particularly useful when the space $X=G / \Lambda$ is not compact. To deal with this non-compactness issue, we study the random walk on $X$ thanks to a regeneration method, i.e. through the Markov chain given by the first return map in a "suitable subset" of $X$. This method will apply to more general $G$-spaces than $X$. Indeed, we will apply it in section 6.6 to the product $X \times X$. The main input of this method is the exponential $\mu$-recurrence of suitable subsets of $X$. These suitable subsets are the sublevel sets $X_{M}$ of a function $u$ satisfying a contraction property with respect to the averaging operator $A_{\mu}$. This method avoids the use of any other arithmetico-geometric information on $X$.

We will first check in section 6.1 that, uniformly for $x \in X_{M}$, the law of the first return time $\tau_{M, x}$ in $X_{M}$ starting from a point $x \in X$ has a finite exponential moment. We will then check in section 6.2 that the laws $\mu_{M, x} \in \mathcal{P}(\Gamma)$ of the corresponding first return cocycle also have a finite exponential moment uniformly for $x$ in $X_{M}$. We will then introduce the averaging operator corresponding to this first return cocycle and study the action of this averaging operator in an unbounded irreducible representation $V$ of the group $\Gamma_{\mu}$. In section 6.3 we will give a uniform lower bound for the average logarithmic growth of a non-zero vector in $V$. We will deduce in section 6.4 that the function on $V$ given by a suitably small negative power of the norm satisfies a contraction property with respect to these averaging operators uniformly for $x$ in $X_{M}$. We will introduce in section 6.5 the notion of "positive $\mu$-unstability" and give a criterion to check this condition. We explain in section 6.6 one of the main applications of positive $\mu$-unstability: we will prove that, when the diagonal is positively $\mu$-unstable, the limit probability measures of any atom-free stationary probability measure do not charge the relative stable leaves. In section 6.7 we explain why, when $\Lambda$ is a lattice, the cusps of $X=G / \Lambda$ are exponentially $\mu$-unstable. Using the exponential $\mu$-unstability of the cusps of $X=G / \Lambda$, we check, in section 6.8 , the criterion which ensures the positive $\mu$-unstability of any $\Gamma$-invariant homogeneous subset of $X=G / \Lambda$.

All the results of this chapter will be used in chapter 7 in order to prove Proposition 7.8 . 
To our knowledge, the first occurrence of exponential $\mu$-recurrence in the context of homogeneous spaces is due to Eskin and Margulis [17. We then repeatedly used this phenomenon without explicitly characterizing it in [4]. The systematic approach which is developed here is greatly influenced by Meyn and Tweedie [22]

\subsection{Exponential $\mu$-recurrence.}

In this section we describe the notion of an exponentially $\mu$-recurrent subset.

Let $G$ be a locally compact second countable group, $\mathcal{G}$ its Borel $\sigma$-algebra, $\mu$ a Borel probability measure on $G$ and $(B, \mathcal{B}, \beta, T)$ the one-sided Bernoulli shift with alphabet $(G, \mathcal{G}, \mu)$.

Let $(X, \mathcal{X})$ be a standard Borel space endowed with a Borel action of $G$. The measurable family of probability measure $X \rightarrow \mathcal{P}(X) ; x \mapsto \mu * \delta_{x}$ induces a Markov operator $A_{\mu}$ on $X$ given by, for every non-negative Borel function $\varphi$ on $X$ and every $x$ in $X$,

$$
A_{\mu} \varphi(x)=\int_{G} \varphi(g x) \mathrm{d} \mu(g) .
$$

Let $Y \subset X$ be a Borel subset. For $x \in X$, we set $\sigma_{Y, x}: B \rightarrow \mathbb{N} \cup\{\infty\}$ to be the first hitting time of $Y$ and $\tau_{Y, x}: B \rightarrow \mathbb{N}_{\geq 1} \cup\{\infty\}$ to be the first return time in $Y$, given by, for $b$ in $B$,

$$
\begin{aligned}
\sigma_{Y, x}(b) & =\inf \left\{n \geq 0 \mid b_{n-1} \cdots b_{0} x \in Y\right\}, \\
\tau_{Y, x}(b) & =\inf \left\{n \geq 1 \mid b_{n-1} \cdots b_{0} x \in Y\right\} .
\end{aligned}
$$

To understand our choices in these definitions, the reader has to keep in mind that, for the trajectory $b$, the " $n^{\text {th }}$-jump" is given by $b_{n-1}$.

A function $\tau: B \rightarrow \mathbb{N} \cup\{\infty\}$ is a stopping time if, for all $0 \leq i<\infty$, the function $\mathbf{1}_{\{\tau=i\}}$ is independent from the family of functions $b_{j}$ with $j \geq i$. For instance, the first hitting time function $\sigma_{Y, x}$ and the first return time function $\tau_{Y, x}$ defined in (6.1) are stopping times.

Definition 6.1. We say that $Y$ is exponentially $\mu$-recurrent if, for some $a_{0}<1$, one has

$$
C_{0}:=\sup _{x \in Y} \int_{B} a_{0}^{-\tau_{Y, x}(b)} \mathrm{d} \beta(b)<\infty .
$$

This means that the first return time in $Y$ has a finite exponential moment uniformly for $x$ in $Y$. We choose such a constant $a_{0}<1$ and introduce the following function:

$$
u_{Y}: X \rightarrow[0, \infty] ; x \mapsto u_{Y}(x)=\int_{B} a_{0}^{-\sigma_{Y, x}(b)} \mathrm{d} \beta(b),
$$

which is an expected exponential of the first hitting time in $Y$.

Lemma 6.2. Let $Y$ be an exponentially $\mu$-recurrent subset of $X$. Then one has the inequality

$$
A_{\mu} u_{Y} \leq a_{0} u_{Y}+C_{0}
$$

Proof. If $x$ is in $Y$, one has $A_{\mu} u_{Y}(x)=\int_{B} a_{0}^{-\tau_{Y, x}(b)} \mathrm{d} \beta(b) \leq C_{0}$.

If $x$ is not in $Y$, one has $\sigma_{Y, b_{0} x}(T b)=\sigma_{Y, x}(b)-1$ for $\beta$-almost every $b$ in $B$, and hence $A_{\mu} u_{Y}(x)=a_{0} u_{Y}(x)$. 
The following proposition is a kind of converse to Lemma 6.2. It gives us a way to construct exponentially $\mu$-recurrent subsets.

Let $u: X \rightarrow[0, \infty]$ be a non-negative Borel function on $X$ and set, for $M<\infty$,

$$
X_{M}:=\{x \in X \mid u(x) \leq M\} .
$$

We assume that there exists $a<1$ and $C>0$ such that

$$
A_{\mu} u \leq a u+C \text {. }
$$

For $x \in X$, we write $\tau_{M, x}:=\tau_{X_{M}, x}$ for the first return time in $X_{M}$.

The following proposition tells us in particular that, for large $M$, the set $X_{M}$ is exponentially $\mu$-recurrent.

Proposition 6.3. Let $G, \mu, B, X, u, a, C$ be as above and satisfy the contraction hypothesis (6.4). Let $a_{0}$ be a constant such that $a<a_{0}<1$ and let $M \geq M_{0}:=$ $\frac{C+1}{a_{0}-a}$. Then, for all $x$ in $X$, one has

$$
\int_{B} a_{0}^{-\tau_{M, x}(b)} \mathrm{d} \beta(b) \leq \max (M, u(x)) .
$$

In particular, if $u(x)$ is finite, then the function $\tau_{M, x}$ is finite $\beta$-almost everywhere on $B$.

The results of this section were inspired by [22, Ch. 15]. The main difference with 22 is that we do not assume " $\varphi$-irreducibility" of the Markov chain, since this property is not satisfied in our applications.

Proof. We fix $x$ in $X$ such that $u(x)<\infty$. We first note that, by an induction argument using (6.4), for all $i \geq 1$, one has

$$
\int_{B} u\left(b_{i-1} \cdots b_{0} x\right) \mathrm{d} \beta(b) \leq a^{i} u(x)+\left(1+a+\cdots+a^{i-1}\right) C .
$$

Hence all the integrals involved in the computations below are finite. To simplify notation, we will write $\tau$ for $\tau_{M, x}$. The main point of the proof is to consider the following averaged weighted Birkhoff sums for $n \geq 1$ :

$$
U_{n}(x):=\int_{B} \sum_{1 \leq i \leq \min (\tau, n)} a_{0}^{1-i} u\left(b_{i-1} \cdots b_{0} x\right) \mathrm{d} \beta(b) .
$$

These sums can be rewritten as

$$
U_{n}(x)=\sum_{i=1}^{n} a_{0}^{1-i} \int_{\{\tau \geq i\}} u\left(b_{i-1} \cdots b_{0} x\right) \mathrm{d} \beta(b) .
$$

Since the first hitting time is a stopping time, the function $\mathbf{1}_{\{\tau \geq i\}}$ is independant from $b_{i-1}$. Integrating first in $b_{i-1}$ and using (6.4), one gets

$$
\begin{aligned}
U_{n}(x) & =\sum_{i=1}^{n} a_{0}^{1-i} \int_{\{\tau \geq i\}}\left(A_{\mu} u\right)\left(b_{i-2} \cdots b_{0} x\right) \mathrm{d} \beta(b) \\
& \leq \sum_{j=0}^{n} a_{0}^{-j} \int_{\{\tau \geq j+1\}}\left(a u\left(b_{j-1} \cdots b_{0} x\right)+C\right) \mathrm{d} \beta(b) .
\end{aligned}
$$

As $M \geq \frac{C+1}{a_{0}-a}$, one has

$$
a u(y)+C \leq a_{0} \max (u(y), M)-1,
$$


hence

$$
U_{n}(x) \leq a_{0} \max (u(x), M)-1+\sum_{j=1}^{n} a_{0}^{-j} \int_{\{\tau \geq j+1\}}\left(a_{0} u\left(b_{j-1} \cdots b_{0} x\right)-1\right) \mathrm{d} \beta(b)
$$

and

$$
U_{n}(x) \leq a_{0} \max (u(x), M)+U_{n}(x)-\sum_{j=0}^{n} a_{0}^{-j} \beta(\{\tau \geq j+1\}) .
$$

Therefore, substracting $U_{n}(x)$ from both sides and letting $n$ go to infinity, one gets

$$
\sum_{j=0}^{\infty} a_{0}^{-j} \beta(\{\tau \geq j+1\}) \leq a_{0} \max (u(x), M) .
$$

In particular, since $u(x)$ is finite, $\tau$ is finite $\beta$-almost everywhere and one has

$$
\int_{B} \frac{a_{0}^{-\tau}-1}{a_{0}^{-1}-1} \mathrm{~d} \beta \leq a_{0} \max (u(x), M),
$$

and hence

$$
\int_{B} a_{0}^{-\tau(b)} \mathrm{d} \beta(b) \leq \max (u(x), M)
$$

as required.

Remark 6.4. Similarly we could say that $Y$ is linearly $\mu$-recurrent if one has $\sup _{x \in Y} \int_{B} \tau_{Y, x}(b) \mathrm{d} \beta(b)<\infty$. By the same method as above, one could characterize the linearly $\mu$-recurrent subsets $Y$ of $X$ as being the sublevel sets of the non-negative functions $u: X \rightarrow[0, \infty]$ satisfying $A_{\mu} u \leq u-1+C \mathbf{1}_{Y}$ for some constant $C>0$ (see [22, Ch. 11]). But this property would not be strong enough to prove unstability results as in section 6.5 below.

\subsection{Law of the first return cocycle.}

In this section, we give a sufficient condition for the existence of finite exponential moments for the law of the first return cocycle.

We keep the notation of section 6.1. Let $Y$ be a subset of $X$. For any points $x$ in $X$ and $b$ in $B$ such that the first return time $\tau(b)=\tau_{Y, x}(b)$ is finite, we define the first return cocycle to be the product $b_{\tau-1} \cdots b_{0}$.

For a point $x \in X$ such that $\beta\left(\left\{b \in B \mid \tau_{Y, x}(b)<\infty\right\}\right)=1$, we set

Definition 6.5. The laws of the first return cocycle are the probability measures $\mu_{Y, x} \in \mathcal{P}(G)$ which are the image of $\beta$ by the first return cocycle $B \rightarrow G ; b \mapsto$ $b_{\tau-1} \cdots b_{0}$. In other words, $\mu_{Y, x}=\int_{B} \delta_{b_{\tau-1} \cdots b_{0}} \mathrm{~d} \beta(b)$.

Hence, the Markov operator $A_{Y}$ describing the first return on this set $Y$ for our random walk on $X$ is the Markov operator given by the transition probabilities $Y \rightarrow \mathcal{P}(Y) ; x \mapsto \mu_{Y, x} * \delta_{x}$. When $Y=X_{M}$ as in section 6.1, we will write $A_{M}:=$ $A_{X_{M}}$.

Definition 6.6. A submultiplicative function on $G$ is a Borel function $N: G \rightarrow$ $[1, \infty)$ such that

$$
N\left(g_{1} g_{2}\right) \leq N\left(g_{1}\right) N\left(g_{2}\right) \text { for all } g_{1}, g_{2} \text { in } G \text {. }
$$


For example, when $\rho: G \rightarrow \mathrm{GL}(V)$ is a continuous finite-dimensional representation of $G$ in a $\mathbb{Q}_{p}$-vector space $V$, where $p$ is a prime number or $p=\infty$, and when $\|$.$\| is an algebra norm on \operatorname{End}(V)$, the function $g \mapsto N(g)=\|\rho(g)\|+\left\|\rho\left(g^{-1}\right)\right\|$ is submultiplicative.

Given a submultiplicative function on $G$, we will say that $\mu$ has finite exponential moments (with respect to $N$ ) if there exists $\delta>0$ such that

$$
\int_{G} N(g)^{\delta} \mathrm{d} \mu(g)<\infty .
$$

The following proposition tells us that, if $\mu$ has finite exponential moments, the laws of the first return cocycle in an exponentially $\mu$-recurrent subset $Y$ of $X$ also have finite exponential moments uniformly for $x \in Y$.

Proposition 6.7. Let $G$ be a locally compact second countable group, $N$ a submultiplicative function on $G$ and $\mu$ a Borel probability measure on $G$ with finite exponential moments with respect to $N$ as in (6.5). Let $X$ be a standard Borel space endowed with a Borel action of $G$ and $Y$ be an exponentially $\mu$-recurrent subset of $X$. Then there exists $\delta>0$ such that

$$
\sup _{x \in Y} \int_{G} N(g)^{\delta} \mathrm{d} \mu_{Y, x}(g)<\infty .
$$

Remark 6.8. We will apply this proposition to the sublevel sets $Y=X_{M}$ of a function $u$ on $X$ satisfying the contraction hypothesis (6.4) when $M$ is large enough.

Proof. Just apply the following technical lemma to the functions $\tau=\tau_{Y, x}$, using the definition of $\mu_{Y, x}$ and the fact that $Y$ is exponentially $\mu$-recurrent.

Lemma 6.9. Let $G$ be a locally compact second countable group, $\mathcal{G}$ its Borel $\sigma$ algebra, $N: G \rightarrow[1, \infty)$ a submultiplicative function on $G$ and $\mu$ a Borel probability measure on $G$ with a finite exponential moment

$$
\int_{G} N(g)^{\delta_{0}} \mathrm{~d} \mu(g) \leq I_{0}<\infty
$$

for some constants $\delta_{0}>0$ and $I_{0} \geq 1$. Let $(B, \mathcal{B}, \beta, T)$ be the one-sided Bernoulli shift with alphabet $(G, \mathcal{G}, \mu)$ and let $\tau: B \rightarrow \mathbb{N}_{\geq 1}$ be a Borel function with a finite exponential moment

$$
\int_{B} e^{t_{0} \tau(b)} \mathrm{d} \beta(b) \leq D_{0}<\infty
$$

for some constants $t_{0}>0$ and $D_{0} \geq 1$.

Then there exists $\delta=\delta\left(\delta_{0}, I_{0}, t_{0}\right)>0$ and $D=D\left(\delta_{0}, I_{0}, t_{0}, D_{0}\right) \geq 1$ such that

$$
\int_{B} N\left(b_{\tau(b)-1} \cdots b_{0}\right)^{\delta} \mathrm{d} \beta(b) \leq D<\infty .
$$

Proof. We will choose

$$
\delta=\frac{t_{0} \delta_{0}}{2\left(t_{0}+\log I_{0}\right)} \quad \text { and } \quad D=\frac{D_{0}^{1-\delta / \delta_{0}}}{e^{t_{0} / 2}-1} .
$$

We want to dominate the integral $I_{\delta}:=\int_{B} N\left(b_{\tau(b)-1} \cdots b_{0}\right)^{\delta} \mathrm{d} \beta(b)$. By (6.7), one has the upper bound, for every $k \geq 1$,

$$
\beta(\{\tau=k\}) \leq D_{0} e^{-k t_{0}} .
$$


Hence, successively using Hölder inequality, the submultiplicativity of $N$ and majoration (6.6), one gets

$$
\begin{aligned}
I_{\delta} & =\sum_{k=1}^{\infty} \int_{B} \mathbf{1}_{\{\tau=k\}}(b) N\left(b_{k-1} \cdots b_{0}\right)^{\delta} \mathrm{d} \beta(b) \\
& \leq \sum_{k=1}^{\infty} \beta(\{\tau=k\})^{1-\delta / \delta_{0}}\left(\int_{B} N\left(b_{k-1} \cdots b_{0}\right)^{\delta_{0}} \mathrm{~d} \beta(b)\right)^{\delta / \delta_{0}} \\
& \leq \sum_{k=1}^{\infty}\left(D_{0} e^{-k t_{0}}\right)^{1-\delta / \delta_{0}} I_{0}^{k \delta / \delta_{0}}=D_{0}^{1-\delta / \delta_{0}} \sum_{k=1}^{\infty} e^{-k t_{0} / 2}=D .
\end{aligned}
$$

This completes the proof of Lemma 6.9.

\subsection{Growth of the first return cocycle.}

In this section we give a lower bound for the expected logarithmic growth of a vector under the action of a random walk on the linear group up to some integrable stopping time.

Let $G$ be a locally compact second countable group, $\mathcal{G}$ its Borel $\sigma$-algebra, $\rho$ : $G \rightarrow \mathrm{GL}(V)$ a continuous finite dimensional representation of $G$ in a normed $\mathbb{Q}_{p^{-}}$ vector space $V$, where $p$ is a prime number or $\infty$, and let $N$ be the submultiplicative function $N: G \rightarrow[1, \infty) ; g \mapsto N(g):=\|\rho(g)\|+\left\|\rho\left(g^{-1}\right)\right\|$. Let $\mu$ be a Borel probability measure on $G$ with finite first moment:

$$
L:=\int_{G} \log N(g) \mathrm{d} \mu(g)<\infty .
$$

We set

$$
L_{0}=L_{0}(\mu):=\inf _{w \in V \backslash 0} \int_{G} \log \frac{\|g w\|}{\|w\|} \mathrm{d} \mu(g) \geq-L .
$$

Let $(B, \mathcal{B}, \beta, T)$ be the one-sided Bernoulli shift whose alphabet is the space $(G, \mathcal{G}, \mu)$. For any Borel function $\tau: B \rightarrow \mathbb{N}_{\geq 1}$ we let, as in Definition 6.5, $\mu_{\tau} \in$ $\mathcal{P}(G)$ be the image of $\beta$ by the map $b \mapsto b_{\tau(b)-1} \cdots b_{0}$. We recall that $\tau$ is a stopping time if, for all $i$, the function $\mathbf{1}_{\{\tau=i\}}$ is independent from the family of functions $b_{j}$ with $j \geq i$.

Lemma 6.10. We assume that $\mu \in \mathcal{P}(G)$ has finite first moment and that $\tau: B \rightarrow$ $\mathbb{N}$ is a $\beta$-integrable stopping time. Then $\mu_{\tau}$ has finite first moment, i.e. one has $\int_{G} \log N(g) \mathrm{d} \mu_{\tau}(g)<\infty$. Moreover, for all $v \in V \backslash 0$, one has

$$
\int_{G} \log \frac{\|g v\|}{\|v\|} \mathrm{d} \mu_{\tau}(g) \geq L_{0} \int_{B} \tau \mathrm{d} \beta
$$

In section 6.4 we will use Lemma 6.10 with $\tau$ equal to a first return time and with $L_{0}>0$. In this case, one has $\tau \geq 1$, and the right-hand side of (6.9) is bounded below by $L_{0}>0$. 
Proof. The proof is a variation of Wald's Lemma. Since $\tau$ is a stopping time, the functions $\mathbf{1}_{\{\tau \geq i\}}$ and $b_{i-1}$ are independent. Hence, one has

$$
\begin{aligned}
\int_{G} \log N(g) \mathrm{d} \mu_{\tau}(g) & =\int_{B} N\left(b_{\tau(b)-1} \cdots b_{0}\right) \mathrm{d} \beta(b) \\
& \leq \int_{B} \sum_{i=1}^{\tau(b)} \log N\left(b_{i-1}\right) \mathrm{d} \beta(b) \\
& =\sum_{i=1}^{\infty} \int_{\{\tau \geq i\}} \log N\left(b_{i-1}\right) \mathrm{d} \beta(b) \\
& =L \sum_{i=1}^{\infty} \beta(\{\tau \geq i\})=L \int_{B} \tau \mathrm{d} \beta<\infty,
\end{aligned}
$$

since $\tau$ is $\beta$-integrable.

This also proves that the left-hand side $M_{\infty}$ of (6.9) is well defined and, by the dominated convergence theorem, that, as $n \rightarrow \infty$, it is the limit of $M_{n}$, where

$$
M_{n}:=\int_{\{\tau \leq n\}} \log \frac{\left\|b_{\tau(b)-1} \cdots b_{0} v\right\|}{\|v\|} \mathrm{d} \beta(b) .
$$

As above one has

$$
M_{n}=\sum_{i=1}^{n} \int_{\{\tau \geq i\}} \log \frac{\left\|b_{i-1} \cdots b_{0} v\right\|}{\left\|b_{i-2} \cdots b_{0} v\right\|} \mathrm{d} \beta(b) .
$$

Again using the independance of $\mathbf{1}_{\{\tau \geq i\}}$ and $b_{i-1}$, integrating first in $b_{i-1}$ and using (6.8) with $g=b_{i-1}$ and $w=b_{i-2} \cdots b_{0} v$, one gets

$$
M_{n} \geq \sum_{i=1}^{n} L_{0} \beta(\{\tau \geq i\})=L_{0} \int_{B} \min (\tau, n) \mathrm{d} \beta .
$$

Hence, letting $n$ go to infinity, one gets $M_{\infty} \geq L_{0} \int_{B} \tau \mathrm{d} \beta$, as required.

Remark 6.11. If $V$ is a representation of $G$ such that the subgroup spanned by the image of $\operatorname{supp}(\mu)$ has semisimple Zariski closure $H$ in $\operatorname{GL}(V)$ and has unbounded action in any $H$-submodule of $V$, by Furstenberg and Kesten's Theorem on the positivity of the first Lyapounov exponent (see Proposition 4.4), there exists $n_{0} \geq 1$ such that, for all $n \geq n_{0}$, the constant $L_{0}\left(\mu^{* n}\right)$ is positive; i.e. if we replace $\mu$ by a suitable power $\mu^{* n}$ we may assume that $L_{0}>0$.

\subsection{Contraction property for the first return cocycle.}

In this section, we first recall the contraction property for linear random walks. Then we prove that this contraction property is also satisfied for the law of the first return cocycle associated to an exponentially $\mu$-recurrent subset.

Let $G$ be a locally compact second countable group and $\rho: G \rightarrow \operatorname{GL}(V)$ a continuous finite-dimensional representation of $G$ in a normed $\mathbb{Q}_{p}$-vector space $V$, where $p$ is either a prime number or $\infty$. Set $\varphi$ to be the function $\varphi: V \backslash 0 \rightarrow$ $\mathbb{R}^{*} ; v \mapsto\|v\|^{-1}$ and $N$ to be the submultiplicative function $N: G \rightarrow[1, \infty) ; g \mapsto$ $N(g):=\|\rho(g)\|+\left\|\rho\left(g^{-1}\right)\right\|$. 
Lemma 6.12. Let $\mu$ be a Borel probability measure on $G$ with finite exponential moments

$$
\int_{G} N(g)^{\delta_{0}} \mathrm{~d} \mu(g) \leq I_{0}<\infty
$$

and with

$$
\inf _{w \in V \backslash 0} \int_{G} \log \frac{\|g w\|}{\|w\|} \mathrm{d} \mu(g) \geq L_{0}>0
$$

for some constants $\delta_{0}>0, I_{0} \geq 1$ and $L_{0}>0$.

Then there exists $\delta_{1}=\delta_{1}\left(\delta_{0}, I_{0}, L_{0}\right)$ such that, for any $\delta \in\left(0, \delta_{1}\right]$, one has, with $a:=1-L_{0} \delta / 2<1$,

$$
A_{\mu}\left(\varphi^{\delta}\right) \leq a \varphi^{\delta}
$$

Proof. This lemma is an extension of Lemma 4.2 of [17]. We will repeat the proof which is based on an asymptotic expansion of order 2 of $e^{-\delta \log (\|g v\| /\|v\|)}$, as $\delta \rightarrow 0$, in order to check that the constants $\delta$ and $a$ can be chosen uniformly for all probability measures $\mu$ satisfying (6.10) and (6.11).

We set $\delta_{1}:=\min \left(\frac{\delta_{0}}{2}, \frac{L_{0} \delta_{0}^{2}}{4 I_{0}}\right)$. We want to dominate the integral

$$
I:=\int_{G} \frac{\|g v\|^{-\delta}}{\|v\|^{-\delta}} \mathrm{d} \mu(g)
$$

To this aim, we use the inequalities, for all $x$ in $\mathbb{R}$,

$$
e^{x} \leq 1+x+\frac{x^{2}}{2} e^{|x|} \text { and } x^{2} \leq e^{|x|},
$$

and we compute

$$
\begin{aligned}
I & =\int_{G} e^{-\delta \log \frac{\|g v\|}{\|v\|}} \mathrm{d} \mu(g) \\
& \leq 1-\delta \int_{G} \log \frac{\|g v\|}{\|v\|} \mathrm{d} \mu(g)+\frac{\delta^{2}}{2} \int_{G}(\log N(g))^{2} N(g)^{\frac{\delta_{0}}{2}} \mathrm{~d} \mu(g) \\
& \leq 1-\delta L_{0}+\frac{2 \delta^{2}}{\delta_{0}^{2}} \int_{G} N(g)^{\delta_{0}} \mathrm{~d} \mu(g)=1-\delta L_{0}+\frac{2 \delta^{2}}{\delta_{0}^{2}} I_{0} \leq 1-\frac{\delta}{2} L_{0},
\end{aligned}
$$

which is the required inequality.

As a corollary, we get the following

Proposition 6.13. Let $\mu$ be a Borel probability measure with finite exponential moments on $G$ and with

$$
\inf _{w \in V \backslash 0} \int_{G} \log \frac{\|g w\|}{\|w\|} \mathrm{d} \mu(g)>0 .
$$

Let $X$ be a standard Borel space endowed with a Borel action of $G$ and $Y$ be an exponentially $\mu$-recurrent subset of $X$. Then for $\delta>0$ small enough, there exists $a_{0}<1$ such that, for all $x$ in $Y$ and all $v \in V$, one has

$$
\int_{G}\|g v\|^{-\delta} \mathrm{d} \mu_{Y, x}(g) \leq a_{0}\|v\|^{-\delta} .
$$

Proof. This is a consequence of Lemma 6.12 applied to all the probability measures $\mu_{Y, x}$ with $x$ in $Y$. Indeed, the uniform upper bound (6.10) for all these probability measures $\mu_{Y, x}$ is given in Proposition 6.7, while the uniform lower bound (6.11) for all these probability measures $\mu_{Y, x}$ is given in Lemma 6.10. 


\subsection{A criterion for positive $\mu$-unstability.}

In this section we introduce the notion of positive $\mu$-unstability and give a handleable criterion for a subset to be positively $\mu$-unstable (Proposition 6.16).

Let $G$ be a locally compact second countable group, $\mu$ be a Borel probability measure on $G$ and $X$ be a locally compact second countable space endowed with a continuous action of $G$.

Definition 6.14. A closed $\Gamma_{\mu}$-invariant subset $Y$ of $X$ is said to be positively $\mu$ unstable if, for every $\varepsilon>0$ and any compact subset $Z \subset X \backslash Y$, there exists a closed subset $F \equiv F_{Z, \varepsilon}$ of $X \backslash Y$ such that, for all $x \in Z$, for all $n \geq 1$, one has

$$
\frac{1}{n} \sum_{0 \leq k<n}\left(\mu^{* k} * \delta_{x}\right)(F) \geq 1-\varepsilon
$$

Here is a straightforward consequence of Definition 6.14

Lemma 6.15. Let $n_{0} \geq 1$. A closed $\Gamma_{\mu}$-invariant subset $Y$ of $X$ is positively $\mu$-unstable if and only if it is positively $\mu^{* n_{0}}$-unstable.

Proof. On the one hand, for a subset $F$ of $X$, and $n \geq 1$, the bound

$$
\frac{1}{n} \sum_{0 \leq k<n}\left(\mu^{* k} * \delta_{x}\right)\left(F^{c}\right) \leq \varepsilon
$$

implies $\frac{1}{n} \sum_{0 \leq k<n}\left(\mu^{* k n_{0}} * \delta_{x}\right)\left(F^{c}\right) \leq n_{0} \varepsilon$.

On the other hand, let $K$ be a compact subset of $\Gamma_{\mu}$ such that $\mu^{* \ell}(K) \geq 1-\varepsilon$, for all $0 \leq \ell<n_{0}$. For every closed subset $F$ of $X \backslash Y, K F$ is also a closed subset of $X \backslash Y$ and, for every probability measure $\nu$ on $X$,

$$
\frac{1}{n_{0}} \sum_{0 \leq \ell<n_{0}}\left(\mu^{* \ell} * \nu\right)(K F) \geq(1-\varepsilon) \nu(F) .
$$

The results follows by applying this inequality to the probability measure $\nu=$ $\frac{1}{n} \sum_{0 \leq k<n} \mu^{* k n_{0}} * \delta_{x}$.

For any Borel function $u: X \rightarrow[0, \infty]$ satisfying $A_{\mu} u \leq a u+C$ for some $a<1, C>0$, we set, for $M<\infty$, as in (6.3), $X_{M}:=\{x \in X \mid u(x) \leq M\}$ and $A_{M}:=A_{X_{M}}$ the first return Markov operator on $X_{M}$; i.e., the Markov operator with transition probabilities $x \mapsto \mu_{X_{M}, x} * \delta_{x}$ as in Definition 6.5

Proposition 6.16. Assume that, for any compact subset $Z \subset X$, there exists a lower semicontinuous function $u: X \rightarrow[0, \infty]$ which is bounded on $Z$ and such that

$$
A_{\mu} u \leq a u+C
$$

for some constants $a<1, C>0$. Let $A_{M}:=A_{X_{M}}$ be the first return Markov operator on the sublevel sets $X_{M}:=\{x \in X \mid u(x) \leq M\}$.

Let $Y$ be a closed $\Gamma_{\mu}$-invariant subset of $X$. Assume that, when $M$ is large enough, for any compact subset $Z \subset X_{M} \backslash Y$, there exists a lower semicontinuous function $v_{M}: X_{M} \rightarrow[0, \infty]$ which is bounded on $Z$, infinite on $Y \cap X_{M}$ and such that

$$
A_{M} v_{M} \leq a_{M} v_{M}+C_{M}
$$

for some constants $a_{M}<1, C_{M}>0$.

Then $Y$ is positively $\mu$-unstable. 
Proof. We denote as usual by $(B, \mathcal{B}, \beta, T)$ the one-sided Bernoulli shift with alphabet $(G, \mathcal{G}, \mu)$, and we recall that the operator $A_{M}$ is given by, for any non-negative Borel function $\varphi$ on $X_{M}$ and any $x$ in $X_{M}$,

$$
\left(A_{M} \varphi\right)(x)=\int_{B} \varphi\left(b_{\tau(b)-1} \cdots b_{0} x\right) \mathrm{d} \beta(b),
$$

where $\tau=\tau_{M, x} \geq 1$ is the first return time in $X_{M}$.

We will prove the following statement, which in view of our assumptions, implies the result: for any $\varepsilon>0$ and $M_{0}>0$, there exists $M>0$ such that, for any $M_{0}^{\prime}>0$ there exists $M^{\prime}>0$ such that, for any $x \in X_{M_{0}}$ with $v_{M}(x) \leq M_{0}^{\prime}$ and any $n \geq 1$, inequality (6.13) is satisfied with

$$
F=X_{M, M^{\prime}}=\left\{y \in X_{M} \mid v_{M}(y) \leq M^{\prime}\right\} .
$$

We will choose

$$
M \geq \frac{16\left(M_{0}+C\right)}{(1-a) \varepsilon^{2}} \quad \text { and } \quad M^{\prime} \geq \frac{16\left(M_{0}^{\prime}+C_{M}\right)}{\left(1-a_{M}\right) \varepsilon^{2}} .
$$

We will first use inequality (6.14) to control the density of the visits in $X_{M}$. For $x$ in $X_{M_{0}}$ and $n \geq 1$, one has

$$
\frac{1}{n} \sum_{k=0}^{n-1}\left(A_{\mu}^{k} u\right)(x) \leq \frac{1}{(1-a) n} u(x)+\frac{C}{1-a} \leq \frac{M_{0}+C}{(1-a)} \leq \varepsilon^{2} M / 16,
$$

which can be rewritten as

$$
\int_{B} \frac{1}{n} \sum_{k=0}^{n-1} u\left(b_{k-1} \cdots b_{0} x\right) \mathrm{d} \beta(b) \leq \varepsilon^{2} M / 16 .
$$

By applying Chebyshev's inequality twice, from this we get

$$
\beta\left(\left\{b \in B \mid \# I_{M}^{n}(b) \geq(1-\varepsilon / 4) n\right\}\right) \geq 1-\varepsilon / 4,
$$

where

$$
I_{M}^{n}(b):=\left\{k \in[0, n-1] \mid u\left(b_{k-1} \cdots b_{0} x\right) \leq M\right\} .
$$

If, for $t \geq 0$, we set $\lceil t\rceil$ for the smallest integer $p \geq t$ and we denote by $\tau_{M, x}^{t}(b)$ the $\lceil t\rceil^{\text {th }}$-return time in $X_{M}$ starting from $x$ on the trajectory $b$, we can rewrite (6.5) as

$$
\beta\left(\left\{b \in B \mid \tau_{M, x}^{(1-\varepsilon / 4) n}(b)<n\right\}\right) \geq 1-\varepsilon / 4 .
$$

Following similar computations, we will now use inequality 6.15 to control the density of the visits in $X_{M, M^{\prime}}$ among the return times in $X_{M}$. Since $v_{M}(x) \leq M_{0}^{\prime}$, for $n \geq 1$, one has

$$
\frac{1}{n} \sum_{k=0}^{n-1}\left(A_{M}^{k} v_{M}\right)(x) \leq \frac{1}{\left(1-a_{M}\right) n} v_{M}(x)+\frac{C_{M}}{1-a_{M}} \leq \varepsilon^{2} M^{\prime} / 16,
$$

which can also be rewritten as

$$
\int_{B} \frac{1}{n} \sum_{k=0}^{n-1} v_{M}\left(b_{\tau_{M, x}^{k}(b)-1} \cdots b_{0} x\right) \mathrm{d} \beta(b) \leq \varepsilon^{2} M^{\prime} / 16 .
$$

Again by Chebyshev's inequality, this gives

$$
\beta\left(\left\{b \in B \mid \# J_{M, M^{\prime}}^{n}(b) \geq(1-\varepsilon / 4) n\right\}\right) \geq 1-\varepsilon / 4,
$$


where

$$
J_{M, M^{\prime}}^{n}(b):=\left\{k \in[0, n-1] \mid v_{M}\left(b_{\tau_{M, x}^{k}(b)-1} \cdots b_{0} x\right) \leq M^{\prime}\right\} .
$$

By (6.16) and (6.17), the set of $b$ in $B$ such that

$$
\tau_{M, x}^{(1-\varepsilon / 4) n}(b)<n \quad \text { and } \quad \# J_{M, M^{\prime}}^{\lceil(1-\varepsilon / 4) n\rceil}(b) \geq(1-\varepsilon / 4)\lceil(1-\varepsilon / 4) n\rceil
$$

has measure $\geq 1-\varepsilon / 2$. Thus, setting

$$
K_{M, M^{\prime}}^{n}(b):=\left\{k \in[0, n-1] \mid b_{k-1} \cdots b_{0} x \in X_{M, M^{\prime}}\right\},
$$

we get

$$
\beta\left(\left\{b \in B \mid \# K_{M, M^{\prime}}^{n}(b) \geq(1-\varepsilon / 2) n\right\}\right) \geq 1-\varepsilon / 2,
$$

from which we deduce

$$
\frac{1}{n} \sum_{k=0}^{n-1}\left(\mu^{* k} * \delta_{x}\right)\left(X_{M, M^{\prime}}\right) \geq(1-\varepsilon / 2)^{2} \geq 1-\varepsilon,
$$

which is the required inequality (6.13).

\subsection{Measure of the relative stable leaves.}

In this section we prove that, for a $G$-space $X$, the positive $\mu$ unstability of the diagonal in $X \times X$ ensures that the limit probability measures $\nu_{b}$ of any atom-free stationary probability measure $\nu$ on $X$ do not charge the relative stable leaves.

Let $G$ be a locally compact second countable group, $\mathcal{G}$ its Borel $\sigma$-algebra, $\mu$ a Borel probability measure on $G, \Gamma_{\mu}$ the smallest closed subgroup of $G$ containing the support of $\mu$ and $(B, \mathcal{B}, \beta, T)$ the one-sided Bernoulli shift with alphabet $(G, \mathcal{G}, \mu)$.

Let $X$ be a locally compact second countable space endowed with a continuous action of $G$. Let $\nu$ be a Borel probability measure on $X$. We recall that $\nu$ is said to be atom-free if, for every $x$ in $X$, one has $\nu(\{x\})=0$. We assume that $\nu$ is $\mu$-stationary, i.e. that $\mu * \nu=\nu$.

Let us denote by $b \mapsto \nu_{b}$ the family of limit probability measures of $\nu$ so that, for every non-negative Borel function $f$ on $X$ and $\beta$-almost every $b$ in $B$, one has $\int_{X} f \mathrm{~d} \nu_{b}=\lim _{n \rightarrow \infty} \int_{X} f \mathrm{~d}\left(b_{0} \cdots b_{n}\right)_{*} \nu$. The existence of this family is due to Furstenberg, and, for $\beta$-almost every $b$ in $B$, one has $\nu_{b}=b_{0 *} \nu_{T b}$ (see [4, Sect. $3.2])$.

Here is a criterion which ensures that these limit probability measures are atomfree. This criterion reinforces [4, Prop. 3.9].

Proposition 6.17 (The $\nu_{b}$ 's are atom-free). Let $G$ be a locally compact second countable group, $X$ be a locally compact second countable space endowed with a continuous action of $G$ and $\mu$ be a Borel probability measure on $G$. Assume that the diagonal $\Delta_{X}$ of $X \times X$ is positively $\mu$-unstable.

Then, for any atom-free $\mu$-stationary Borel probability measure $\nu$ on $X$, for $\beta$ almost every $b$ in $B$, the probability measure $\nu_{b}$ is also atom-free.

Proof of Proposition 6.17, We begin the proof with a special case.

First step. If, for $\beta$ almost every $b, \nu_{b}$ is a Dirac mass, then so is $\nu$.

We assume in this first step that, for $\beta$-almost every $b$ in $B$, the probability measure $\nu_{b}$ is a Dirac mass $\delta_{\kappa(b)}$ where $\kappa: B \rightarrow X$ is a $\mathcal{B}$-measurable map. We will then prove by contradiction that $\nu$ is a Dirac mass. To get a contradiction, we study 
the random walk associated to $\mu$ on $X \times X$. We will use the existence of $\kappa$ and the Chacon-Ornstein ergodic theorem to see that this random walk converges towards the diagonal $\Delta_{X}$, which contradicts the positive $\mu$-instability of the diagonal. To make this precise, we need more notation.

For $g$ in $G$ and $b=\left(b_{0}, b_{1}, \ldots\right)$ in $B$, we set $g b=\left(g, b_{0}, b_{1}, \ldots\right)$. One then has, for $\mu$-almost every $g$ in $G$ and $\beta$-almost every $b$ in $B, \kappa(g b)=g \kappa(b)$.

We endow $B=G^{\mathbb{N}}$ with the product topology. According to Lusin's theorem, for every $\varepsilon>0$ there exists a compact subset $K \subset B$ such that $\beta(K) \geq 1-\varepsilon$ and the restriction of $\kappa$ to $K$ is continuous.

We introduce the transfer operator $L_{\mu}$ on $B$ given by, for $\varphi$ in $\mathrm{L}^{1}(B, \beta)$ and for $\beta$-almost every $b$ in $B$,

$$
\left(L_{\mu} \varphi\right)(b)=\int_{G} \varphi(g b) d \mu(g) .
$$

Since $L_{\mu}$ is the adjoint of the shift $T$, it is ergodic. The Chacon-Ornstein ergodic theorem [14 applied to the function $\varphi=\mathbf{1}_{K}$ tells us that, for $b$ outside a $\beta$-null subset $N \subset B$, one has the equality

$$
\lim _{n \rightarrow \infty} \frac{1}{n} \sum_{n \leq k<2 n}\left(L_{\mu}^{k} \mathbf{1}_{K}\right)(b)=\beta(K) \geq 1-\varepsilon .
$$

Enlarging the $\beta$-null set $N$, we may also assume that, for every $b$ in $B \backslash N$, for every integer $k \geq 0$, and for $\mu^{\otimes k}$-almost every $\left(g_{1}, \ldots, g_{k}\right)$ in $G^{k}$, one has $\kappa\left(g_{1} \cdots g_{k} b\right)=$ $g_{1} \cdots g_{k} \kappa(b)$.

Assume by contradiction that $\nu$ is not a Dirac mass. Then, as $\nu=\kappa_{*} \beta$, the set $E:=\left\{\left(b, b^{\prime}\right) \in B \times B \mid \kappa(b) \neq \kappa\left(b^{\prime}\right)\right\}$ is not $\beta \otimes \beta$-null. Hence one can find two points $b$ and $b^{\prime}$ outside $N$ such that the images $x:=\kappa(b)$ and $x^{\prime}:=\kappa\left(b^{\prime}\right)$ are distinct.

Since the diagonal $\Delta_{X}$ is positively $\mu$-unstable, for every $\varepsilon>0$ there exists a closed subset $F_{\varepsilon}$ of $X \times X \backslash \Delta_{X}$ such that, for all $n \geq 1$, one has

$$
\frac{1}{n} \sum_{n \leq k<2 n}\left(\mu^{* k} * \delta_{\left(x, x^{\prime}\right)}\right)\left(F_{\varepsilon}\right) \geq 1-\varepsilon .
$$

Since the restriction of $\kappa$ to $K$ is continuous, one has

$$
\begin{aligned}
& \exists n_{\varepsilon}>0, \forall n \geq n_{\varepsilon}, \forall g_{1}, \ldots, g_{n} \in G \text { such that } g_{1} \cdots g_{n} b \in K \\
& \text { and } g_{1} \cdots g_{n} b^{\prime} \in K, \text { one has }\left(\kappa\left(g_{1} \cdots g_{n} b\right), \kappa\left(g_{1} \cdots g_{n} b^{\prime}\right)\right) \notin F_{\varepsilon} .
\end{aligned}
$$

By (6.18), there exists an integer $n_{0} \geq n_{\varepsilon}$ such that, for every $n \geq n_{0}$,

$$
\begin{aligned}
& \frac{1}{n} \sum_{n \leq k<2 n} \mu^{\otimes k}\left(\left\{\left(g_{1}, \ldots, g_{k}\right) \in G^{k} \mid g_{1} \cdots g_{k} b \in K\right\}\right) \geq 1-\varepsilon, \\
& \frac{1}{n} \sum_{n \leq k<2 n} \mu^{\otimes k}\left(\left\{\left(g_{1}, \ldots, g_{k}\right) \in G^{k} \mid g_{1} \cdots g_{k} b^{\prime} \in K\right\}\right) \geq 1-\varepsilon .
\end{aligned}
$$

Hence, using (6.20), one gets

$$
\frac{1}{n} \sum_{n \leq k<2 n} \mu^{\otimes k}\left(\left\{\left(g_{1}, \ldots, g_{k}\right) \in G^{k} \mid\left(g_{1} \cdots g_{k} x, g_{1} \cdots g_{k} x^{\prime}\right) \notin F_{\varepsilon}\right\}\right) \geq 1-2 \varepsilon .
$$

This gives a contradiction with (6.19) as soon as $\varepsilon<\frac{1}{3}$. Hence $\nu$ is a Dirac mass.

Second step. We will reduce the general case to the first one. 
We assume that the set $D:=\left\{b \in B \mid \nu_{b}\right.$ has atoms $\}$ satisfies $\beta(D)>0$, and we shall prove that $\nu$ has atoms. Since $\nu_{b}=b_{0 *} \nu_{T b}$, this set $D$ is $T$-invariant. Since $\beta$ is $T$-ergodic, one has $\beta(D)=1$. The same argument also shows that the maximal mass $m_{b}$ of the atoms of $\nu_{b}$ is $\beta$-almost everywhere constant on $B$ and that the number $N_{b}$ of atoms of $\nu_{b}$ whose mass is $m_{b}$ is also $\beta$-almost everywhere constant. We set $m_{0}>0$ for this mass and $N_{0} \geq 1$ for this number of atoms. Let $\nu_{b}^{\prime}$ be the probability measure which is equidistributed on these $N_{0}$ atoms of $\nu_{b}$ whose mass is $m_{0}$. One also has $\nu_{b}^{\prime}=b_{0 *} \nu_{T b}^{\prime}$. Hence the probability $\nu^{\prime}$ on $X$ given by $\nu^{\prime}:=\int_{B} \nu_{b}^{\prime} \mathrm{d} \beta(b)$ is also $\mu$-stationary, and one can write $\nu$ as the sum of $m_{0} N_{0} \nu^{\prime}$ and of a $\mu$-stationary measure with total mass $\left(1-N_{0} m_{0}\right)$. By construction, the probability measures $\nu_{b}^{\prime}$ are the limit probability measures of $\nu^{\prime}$, and it suffices to prove that $\nu^{\prime}$ has atoms.

The group $G$ acts naturally on the set $X^{\prime}$ of finite subsets of $X$ with $N_{0}$ elements. The support $x_{b}^{\prime}$ of $\nu_{b}^{\prime}$ is an element of $X^{\prime}$, and the family $b \mapsto \nu_{b}^{\prime \prime}$ of probability measures on $X^{\prime}$ where $\nu_{b}^{\prime \prime}$ is the Dirac mass at $x_{b}^{\prime}$ still satisfies the equality $\nu_{b}^{\prime \prime}=$ $b_{0 *} \nu_{T b}^{\prime \prime}$, for $\beta$-almost all $b$ in $B$. Hence the probability measure $\nu^{\prime \prime}:=\int_{B} \nu_{b}^{\prime \prime} \mathrm{d} \beta(b)$ on $X^{\prime}$ is $\mu$-stationary.

We want to apply the first case to the action of $G$ on $X^{\prime}$. To this aim, we now check that the diagonal $\Delta_{X^{\prime}}$ of $X^{\prime} \times X^{\prime}$ is also positively $\mu$-unstable. Indeed, let $Z^{\prime}$ be a compact subset of $X^{\prime} \times X^{\prime} \backslash \Delta_{X^{\prime}}$ and $\varepsilon>0$. There exists a compact subset $Z$ of $X \times X \backslash \Delta_{X}$ such that

$$
\begin{aligned}
& \text { for all }\left(x, x^{\prime}\right)=\left(\left\{x_{1}, \ldots, x_{N_{0}}\right\},\left\{x_{1}^{\prime}, \ldots, x_{N_{0}}^{\prime}\right\}\right) \in Z^{\prime} \text {, there } \\
& \text { exists } 1 \leq i \leq N_{0} \text { such that, for all } 1 \leq j \leq N_{0} \text {, one has } \\
& \left(x_{i}, x_{j}^{\prime}\right) \in Z \text {. }
\end{aligned}
$$

Let $F$ be a closed subset of $X \times X \backslash \Delta_{X}$ satisfying (6.13) and

$$
F^{\prime}:=\left\{\left(x, x^{\prime}\right) \in Z^{\prime} \mid \exists 1 \leq i \leq N_{0}, \forall 1 \leq j \leq N_{0}, \quad\left(x_{i}, x_{j}^{\prime}\right) \in F\right\} .
$$

Then, for any $\left(x, x^{\prime}\right)$ in $Z^{\prime}$ and $n \geq 1$ one has, choosing $i$ as in (6.21),

$$
\frac{1}{n} \sum_{0 \leq k<n}\left(\mu^{* k} * \delta_{\left(x, x^{\prime}\right)}\right)\left(F^{\prime c}\right) \leq \frac{1}{n} \sum_{1 \leq j \leq N_{0}} \sum_{0 \leq k<n}\left(\mu^{* k} * \delta_{\left(x_{i}, x_{j}^{\prime}\right)}\right)\left(F^{c}\right) \leq N_{0} \varepsilon .
$$

This proves that $\Delta_{X^{\prime}}$ is positively $\mu$-unstable.

According to the first case, the probability measure $\nu^{\prime \prime}$ is a Dirac mass $\delta_{y}$. Hence, $\nu$ has finite support, which should be proved.

Let $B^{X}$ be $B \times X, \mathcal{B}^{X}$ the product $\sigma$-algebra $\mathcal{B} \otimes \mathcal{X}, \beta^{X}$ the probability measure on $B^{X}$ given by $\beta^{X}:=\int_{B} \delta_{b} \otimes \nu_{b} \mathrm{~d} \beta(b)$ and $T^{X}$ the transformation of $B^{X}$ given, for $(b, x)$ in $B^{X}$, by $T^{X}(b, x)=\left(T b, b_{0}^{-1} x\right)$. As in section 3.2 (see also 4, Sect. 3.1]), this transformation preserves $\beta^{X}$.

Let $d$ be a distance on $X$ inducing the topology of $X$. For $(b, x)$ in $B \times X$, set

$$
W_{b}(x)=\left\{x^{\prime} \in X \mid d\left(b_{p}^{-1} \cdots b_{0}^{-1} x, b_{p}^{-1} \cdots b_{0}^{-1} x^{\prime}\right) \underset{p \rightarrow \infty}{\longrightarrow} 0\right\},
$$

which is the relative stable leaf of $(b, x)$. When $X$ is compact, this leaf does not depend on the choice of $d$, but it might in general.

The following proposition, combined with Proposition 6.17 will be used in the course of the proof of Proposition 7.8

Proposition 6.18. Let $G$ be a locally compact second countable group, $X$ be a locally compact second countable metric space endowed with a continuous action 
of $G$ and $\mu$ be a Borel probability measure on $G$. Let $\nu$ be a $\mu$-stationary Borel probability measure on $X$. Let $L$ be a second countable locally compact group acting continuously on $X$. Assume that the actions of $L$ and $\Gamma_{\mu}$ commute. Then, for $\beta^{X}$-almost every $(b, x)$ in $B^{X}$, one has $\nu_{b}\left(L W_{b}(x) \backslash L x\right)=0$.

Proof. Let $R$ be the transformation of $B \times X \times X$ given by, for $\left(b, x, x^{\prime}\right)$ in $B \times X \times X$,

$$
R\left(b, x, x^{\prime}\right)=\left(T b, b_{0}^{-1} x, b_{0}^{-1} x^{\prime}\right) .
$$

The Borel probability measure

$$
\Lambda=\int_{B} \delta_{b} \otimes \nu_{b} \otimes \nu_{b} \mathrm{~d} \beta(b)
$$

on $B \times X \times X$ is $R$-invariant. Write $L=\bigcup_{n \geq 1} L_{n}$, where $\left(L_{n}\right)$ is an exhaustive sequence of compact subsets of $L$. Let us introduce the $R$-invariant subsets

$$
Z=\left\{\left(b, x, x^{\prime}\right) \in B \times X \times X \mid x^{\prime} \in L W_{b}(x) \backslash L x\right\}
$$

and, for $n \geq 1$,

$$
Z_{n}=\left\{\left(b, x, x^{\prime}\right) \in Z \mid x^{\prime} \in L_{n} W_{b}(x)\right\} .
$$

Let $\varphi_{n}$ be the function on $B \times X \times X$ given by

$$
\varphi_{n}\left(b, x, x^{\prime}\right)=\inf _{\ell \in L_{n}} d\left(\ell x, x^{\prime}\right) .
$$

This function $\varphi_{n}$ is non-zero on $Z_{n}$. By definition, for $\Lambda$-almost every $z$ in $Z_{n}$, one has $\varphi_{n}\left(R^{p}(z)\right) \underset{p \rightarrow \infty}{\longrightarrow} 0$. Hence, according to the Poincaré recurrence theorem, one has $\Lambda\left(Z_{n}\right)=0$. Since this is true for all $n \geq 1$, one has $\Lambda(Z)=0$. This proves our claim.

\subsection{Exponential $\mu$-unstability of the cusps.}

Going back to the framework of homogeneous spaces, in this section we give two equivalent definitions for the exponential $\mu$-unstability of the cusps of $X=G / \Lambda$ and we check that they are satisfied for finite volume spaces.

Let $G$ be an $\mathcal{S}$-adic Lie group, $\Lambda$ a discrete subgroup of $G, X=G / \Lambda$ and $\mu \in \mathcal{P}(G)$ an Ad-semisimple Borel probability measure on $G$ with finite exponential moments in $\mathfrak{g}$.

Fix a standard open subset $\Omega$ of $G$. We recall from Definition 2.1 that, for every $x$ in $X$, we denote by $r_{X}(x)$ the injectivity radius in $x$ that is the supremum of the radii $r \leq r_{0}$ for which the map $\mathfrak{g} \rightarrow X ; w \mapsto \exp _{\Omega}(w) x$ is one-to-one on the ball $B(0, r) \subset \mathfrak{g}$.

Lemma 6.19. The following are equivalent:

(i) For every compact subset $K$ of $X$, there exists an exponentially $\mu$-recurrent (see Definition 6.1) closed set $F \subset X$ containing $K$ on which $r_{X}$ is bounded below, i.e. such that $\inf _{x \in F} r_{X}(x)>0$.

(ii) For every compact subset $K$ in $X$, there exists a lower semicontinuous function $u: X \rightarrow[0, \infty]$ which is bounded on $K$ and such that $r_{X}$ is bounded below on every sublevel set $X_{M}:=\{x \in X \mid u(x) \leq M\}$ and there exist constants a $<1$, $C>0$ with $A_{\mu} u \leq a u+C$.

Definition 6.20. When either of the equivalent conditions of Lemma 6.19 is satisfied, we shall say that the cusps of $X$ are exponentially $\mu$-unstable. 
Proof of Lemma 6.19, (ii) $\Rightarrow$ (i) Given a compact set $K$ in $X$, let $u: X \rightarrow[0, \infty]$ be a function as in (ii). We choose $F$ to be a sublevel set $\{x \in X \mid u(x) \leq M\}$ containing $K$. By Proposition 6.3, $F$ is an exponentially $\mu$-recurrent subset. Since $u$ is lower semicontinuous, $F$ is closed and, by assumption, the injectivity radius is bounded below on $F$.

(i) $\Rightarrow$ (ii) Given a compact set $K$ in $X$, let $F \subset X$ be a closed subset as in (i). The injectivity radius $r_{X}$ is bounded below on $F$ and there exists $a<1$ and $C>0$, such that, for all $x \in F$, one has $\int_{B} a^{-\tau_{F, x}(b)} \mathrm{d} \beta(b) \leq C$.

We choose $u: X \rightarrow[0, \infty]$ to be the expected exponential of the first hitting time in $F, u(x)=\int_{B} a^{-\sigma_{F, x}(b)} \mathrm{d} \beta(b)$, as in (6.2). Since $F$ is closed, by Fatou's Lemma, this function $u$ is lower semicontinuous and, by Lemma 6.2. one has $A_{\mu} u \leq a u+C$. In particular, for every $x$ in $X$, one has

$$
\beta\left(\left\{b \in B \mid \exists 0 \leq j \leq n-1 \quad b_{j-1} \cdots b_{0} x \in F\right\}\right) \geq 1-u(x) a^{n} .
$$

Pick $M>0$ and set $X_{M}:=\{x \in X \mid u(x) \leq M\}$. We have to prove that the injectivity radius is bounded below on $X_{M}$. By (6.23), for $n$ large enough, uniformly for $x$ in $X_{M}$, one has

$$
\beta\left(\left\{b \in B \mid \exists 0 \leq j \leq n-1 \quad b_{j-1} \cdots b_{0} x \in F\right\}\right) \geq 1 / 2 .
$$

Now, choose a compact subset $K$ of $G$ such that, for any $0 \leq j \leq n-1$, one has $\mu^{* j}(K)>1-1 / 2 n$. As a consequence, for any $x$ in $X_{M}$, there exists $g$ in $K$ such that $g x \in F$. But by Lemma 5.2 , there exists $\varepsilon>0$ such that, for any $x$ in $X$ with $r_{X}(x)<\varepsilon$ and for any $g$ in $K$, one has $r_{X}(g x) \leq\|\operatorname{Ad} g\| r_{X}(x)$. Since $r_{X}$ is bounded below on $F$, it is also bounded below on $X_{M}$.

When $\Lambda$ is a non-cocompact lattice, exponential $\mu$-unstability of the cusps of $X$ follows from an extension of a result by Eskin and Margulis which constructs a proper function $u$ on $X$ satisfying (6.4). The following lemma was used in section 2.3 to prove that Theorem 2.5 follows from Theorem 2.7 .

Lemma 6.21. Let $G$ be an $\mathcal{S}$-adic Lie group, $\Lambda$ a lattice of $G, X=G / \Lambda$ and $\mu$ an Ad-semisimple probability measure on $G$ which admits finite exponential moments in $\mathfrak{g}$. Then the cusps of $X$ are exponentially $\mu$-unstable.

Proof. According to [7, Proposition 7.3], which extends Eskin-Margulis' main result in [17], given a compact subset $K$ of $X$ there exists a lower semicontinuous function $u: X \rightarrow[0, \infty]$ which is bounded on $K$ such that, for any $M \in[0, \infty)$, the sublevel set $u^{-1}([0, M])$ is compact and there exist constants $a<1, C>0$ with $A_{\mu}(u) \leq$ $a u+C$. The result follows, as the injectivity radius is bounded below on every compact subset of $X$.

\subsection{Positive $\mu$-unstability of homogeneous subspaces.}

In this section we apply the criterion of section 6.5 to prove that the $\Gamma_{\mu}$-invariant homogeneous subsets of $X=G / \Lambda$ are positively $\mu$-unstable.

Let $G$ be an $\mathcal{S}$-adic Lie group, $\Lambda$ be a discrete subgroup of $G$ and $X=G / \Lambda$. As in section 6.7. we fix a standard open subset $\Omega$ of $G$ with exponential map $\exp _{\Omega}: O \rightarrow \Omega$ and we set $r_{X}$ for the injectivity radius in $X$.

Let $Y \subset X$ be a homogeneous closed subset of $X$ and $S=\{g \in G \mid g Y=Y\}$ be the stabilizer of $Y$. We choose a complementary subspace $\mathfrak{t}$ of the Lie algebra of $S$ in the Lie algebra of $G$. We fix $\varepsilon_{0}>0$ such that $O \cap \mathfrak{t}$ contains the closed ball 
with radius $\varepsilon_{0}$ in $\mathfrak{t}$. For every $x$ in $Y$ we set $r_{X, Y}(x)$ to be the transverse injectivity radius at $x$; that is,

$$
r_{X, Y}(x)=\sup \left(\left\{0<\varepsilon \leq \varepsilon_{0} \mid \begin{array}{l|l}
\exp _{\Omega}\left(B_{\mathfrak{t}}(\varepsilon)\right) x \cap Y \text { con- } \\
\text { tains at most one point }
\end{array}\right\}\right) .
$$

Definition 6.22. We will say that $r_{X}$ controls $r_{X, Y}$ if, for every $\eta>0$, there exists $\gamma>0$ such that, for all $x \in Y$ with $r_{X} \geq \eta$, one has $r_{X, Y} \geq \gamma$.

Remark 6.23. This definition does not depend on the choices of $\mathfrak{t}, \Omega$ and $\varepsilon_{0}$.

From Proposition 6.16, we deduce the following general criterion for positive $\mu$-unstability in the homogeneous setting.

Proposition 6.24. Let $G$ be an $\mathcal{S}$-adic Lie group, $\Lambda$ be a discrete subgroup of $G$, $X=G / \Lambda$ and $\mu \in \mathcal{P}(G)$ be an Ad-semisimple probability measure on $G$ such that $H_{\mu}=H_{\mu}^{n c}$, which admits finite exponential moments in $\mathfrak{g}$. We assume that $\left(G, \Gamma_{\mu}\right)$ has good exponentials and that the cusps of $X$ are exponentially $\mu$-unstable (see Definitions 5.9 and 6.20). Let $Y \subset X$ be a $\Gamma_{\mu}$-invariant homogeneous closed subset of $X$ such that $r_{X}$ controls $r_{X, Y}$. Let $K_{L}$ be a compact subset of the centralizer $L$ of $\Gamma_{\mu}$. Then the subset $K_{L} Y \subset X$ is positively $\mu$-unstable.

In particular, $Y$ itself is positively $\mu$-unstable. Before proving this proposition we state two nice corollaries of it.

Corollary 6.25. Let $G$ be a weakly regular $\mathcal{S}$-adic Lie group, $\Lambda$ a lattice of $G$, $X=G / \Lambda$ and $\mu \in \mathcal{P}(G)$ a compactly supported Ad-semisimple probability measure on $G$ with $H_{\mu}=H_{\mu}^{n c}$. Then every $\Gamma_{\mu}$-invariant homogeneous closed subset $Y$ of $X$ is positively $\mu$-unstable.

Proof of Corollary 6.25. We just check that the assumptions of Proposition 6.24 are satisfied.

By Proposition 5.11, since $G$ is weakly regular and $\Gamma_{\mu}$ is compactly generated, $\left(G, \Gamma_{\mu}\right)$ has good exponentials.

Besides $\Lambda$ being a lattice, on one hand, by Lemma 6.21 the cusps of $X$ are exponentially $\mu$-unstable and, on the other hand, for $\eta>0$, the set $X_{\eta}:=\{x \in X \mid$ $\left.r_{X}(x) \geq \eta\right\}$ is compact and the function $r_{X, Y}$ is bounded below on $X_{\eta}$. Hence $r_{X}$ controls $r_{X, Y}$.

Corollary 6.26. Let $G$ be an $\mathcal{S}$-adic Lie group, $\Lambda$ a discrete subgroup of $G, X=$ $G / \Lambda, \mu \in \mathcal{P}(G)$ an Ad-semisimple probability measure on $G$ with $H_{\mu}=H_{\mu}^{n c}$ which admits finite exponential moments in $\mathfrak{g}, K_{L}$ a compact subset of the centralizer $L$ of $\Gamma_{\mu}$ and

$$
\Delta_{X}^{K_{L}}:=\left\{(x, y) \in X \times X \mid \exists \ell \in K_{L} \quad y=\ell x\right\} .
$$

We assume that $\left(G, \Gamma_{\mu}\right)$ has good exponentials and that the cusps of $X$ are exponentially $\mu$-unstable. Then the subset $\Delta_{X}^{K_{L}}$ of $X \times X$ is positively $\mu$-unstable.

Proof of Corollary 6.26. We just have to check that $r_{X \times X}$ controls the transverse spectral radius $r_{X \times X, \Delta_{X}}$. Indeed, choose $\mathfrak{g} \times\{0\}$ as the complementary subspace $\mathfrak{t}$; then, for $(x, y)$ in $X \times X$, one has $r_{X \times X, \Delta_{X}}(x, y) \geq r_{X}(x)$.

Proof of Proposition 6.24. We aim at applying the criterion of Proposition 6.16. Since the cusps of $X$ are exponentially $\mu$-unstable, if $Z$ is some compact subset of $X$, there exists a lower semicontinuous function $u: X \rightarrow[0, \infty]$ such that $A_{\mu} u \leq a u+C$ 
for some constants $a<1, C>0$, which is bounded on $Z$ and such that, on the sublevel sets $X_{M}:=\{x \in X \mid u(x) \leq M\}$, the injectivity radius $r_{X}$ is bounded below. Let $A_{M}:=A_{X_{M}}$ be the first return Markov operator on $X_{M}$.

For any large enough $M>0$ and any compact subset $Z \subset X_{M} \backslash K_{L} Y$, we aim at constructing a function $v_{M}: X_{M} \rightarrow[0, \infty]$ which is bounded on $Z$, infinite on $K_{L} Y \cap X_{M}$ and such that

$$
A_{M} v_{M} \leq a_{M} v_{M}+C_{M}
$$

for some constants $a_{M}<1, C_{M}>0$.

To this aim, we will apply Proposition 6.13 to the subset $X_{M}$, for large enough $M>0$. We first recall that, by Proposition 6.3. $X_{M}$ is exponentially $\mu$-recurrent. We now check that the assumption (6.12) of Proposition 6.13 is satisfied. First, we fix some $\delta_{0}>0$ such that $\int_{G} N(g)^{\delta_{0}} \mathrm{~d} \mu(g)<\infty$, where, as in section 6.3, $N(g)=\|\operatorname{Ad} g\|+\left\|\operatorname{Ad} g^{-1}\right\|$. Let $\mathfrak{l}$ be the Lie algebra of $L$. As $\left(G, \Gamma_{\mu}\right)$ has good exponentials, $\mathfrak{l}$ is the space of $\Gamma_{\mu}$-invariant elements of $\mathfrak{g}$. Let $\mathfrak{v}$ be its unique $\Gamma_{\mu^{-}}$ invariant complementary subspace. Since, by Lemma 6.15, we can replace $\mu$ by a suitable power, we may assume, according to Furstenberg-Kesten's theorem on the positivity of the first Lyapounov exponent (see Proposition 4.4), that the expected logarithmic growth of any non-zero vector in $\mathfrak{v}$ is uniformly bounded below as in (6.11); that is,

$$
\inf _{w \in \mathfrak{v} \backslash 0} \int_{G} \log \frac{\|g w\|}{\|w\|} \mathrm{d} \mu(g)>0 .
$$

By Proposition 6.13, if $\delta<\delta_{0}$ is small enough, there exists $a_{0}<1$ such that, for all $x$ in $X_{M}$ and all $w \in \mathfrak{v}$, one has

$$
\int_{G}\|g w\|^{-\delta} \mathrm{d} \mu_{M, x}(g) \leq a_{0}\|w\|^{-\delta}
$$

By Proposition 6.7, one can assume

$$
I_{M}=\sup _{x \in X_{M}} \int_{G} N(g)^{2 \delta} \mathrm{d} \mu_{M, x}(g)<\infty .
$$

We can now give the formula which defines the functions $v_{M}$ we are looking for. We fix a $\left(G, \Gamma_{\mu}\right)$-good standard open subset $\Omega$ of $G$ and set $O=\log \Omega$. Let $Z$ be a compact subset of $X_{M} \backslash K_{L} Y$. Let $S$ be the stabilizer of $Y$ in $G, \mathfrak{s}$ be its Lie algebra and choose a $\Gamma_{\mu}$-invariant complementary subspace $\mathfrak{t}^{\prime} \subset \mathfrak{v}$ of $\mathfrak{s} \cap \mathfrak{v}$ so that $\mathfrak{g}=(\mathfrak{s}+\mathfrak{l}) \oplus \mathfrak{t}^{\prime}$. Since $r_{X}$ controls $r_{X, Y}$ and $r_{X}$ is bounded below on $X_{M}, r_{X, Y}$ is also bounded below on $X_{M}$. We can find an open neighborhood $U$ of $e$ in $L$ and $\varepsilon>0$ with $B_{\mathfrak{t}^{\prime}}(\varepsilon) \subset O$ such that, for any $x$ in $X_{M}$ and $\ell$ in $L$, there exists at most one $w$ in $\mathfrak{t}$ with $\|w\| \leq \varepsilon$ and $x \in \exp _{\Omega}(w) U \ell Y$. By shrinking $U$, we can assume $Z \cap \bar{U} K_{L} Y=\emptyset$. Pick $\ell_{1}, \ldots, \ell_{r}$ in $K_{L}$ with $K_{L} \subset U \ell_{1} \cup \cdots \cup U \ell_{r}$.

For $x \in X_{M}$ and $1 \leq i \leq r$, set

$$
v_{i}(x)= \begin{cases}\|w\|^{-\delta} & \text { if } x \in e^{w} U \ell_{i} Y \text { with } w \in \mathfrak{t}^{\prime} \text { and }\|w\| \leq \varepsilon \\ \varepsilon^{-\delta} & \text { else }\end{cases}
$$

and $v_{M}(x)=v_{1}(x)+\cdots+v_{r}(x)$.

By construction, the function $v_{M}: X_{M} \rightarrow\left[\varepsilon^{-\delta}, \infty\right]$ is lower semicontinuous, bounded on $Z$ and infinite on $K_{L} Y$. To conclude, we will check that the function $v_{M}$ satisfies inequality (6.24) with $a_{M}=\frac{1+a_{0}}{2}$ and $C_{M}=r R_{M}^{2 \delta} \varepsilon^{-\delta}$, where $R_{M}:=$ $\left(\frac{2 I_{M}}{1-a_{0}}\right)^{\delta^{-1}}$. 
We fix $1 \leq i \leq r$ and $x$ in $X_{M}$. First, let us note that, for any $g$ in $\Gamma_{\mu}$ with $g x \in X_{M}$, we have

$$
v_{i}(g x) \leq N(g)^{\delta} v_{i}(x) .
$$

Indeed, if $v_{i}(g x) \leq N(g)^{\delta} \varepsilon^{-\delta}$, this follows from the fact that $v_{i}(x) \geq \varepsilon^{-\delta}$. Otherwise, one has $g x \in e^{w} U \ell_{i} Y$ with $w \in \mathfrak{t}^{\prime}$ and $\|w\| \leq N(g)^{-1} \varepsilon$, and therefore, as $\Omega$ is $\left(G, \Gamma_{\mu}\right)$-good, $x \in e^{g^{-1} w} U \ell_{i} Y$ and

$$
v_{i}(x)=\left\|g^{-1} w\right\|^{-\delta} \geq N(g)^{-\delta}\|w\|^{-\delta}=N(g)^{-\delta} v_{i}(g x) .
$$

Now, let us decompose the probability measure $\mu_{M, x}$ on $G$ as a sum $\mu_{M, x}=$ $\mu_{1}+\mu_{2}$ where

$$
\mu_{1}=\mathbf{1}_{\left\{N(g) \leq R_{M}\right\}} \mu \text { and } \mu_{2}=\mathbf{1}_{\left\{N(g)>R_{M}\right\}} \mu .
$$

We will first dominate $A_{\mu_{1}} v_{i}$. On one hand, if $v_{i}(x) \leq R_{M}^{\delta} \varepsilon^{-\delta}$, one has, by (6.27), $\left(A_{\mu_{1}} v_{i}\right)(x) \leq R_{M}^{2 \delta} \varepsilon^{-\delta}$. On the other hand, if $v_{i}(x) \geq R_{M}^{\delta} \varepsilon^{-\delta}$, then, writing $x \in e^{w} U \ell_{i} Y$, with $w \in \mathfrak{t}^{\prime},\|w\| \leq R_{M}^{-1} \varepsilon$, one has again, for all $g$ in $\Gamma_{\mu}$ with norm $\leq R_{M}$ and $g x \in X_{M}, g x \in e^{g w} U \ell_{i} Y$, and hence $v_{i}(g x)=\|g w\|^{-\delta}$ and, according to (6.25),

$$
\left(A_{\mu_{1}} v_{i}\right)(x) \leq a_{0}\|w\|^{-\delta}=a_{0} v_{i}(x) .
$$

In both cases, one has the upper bound

$$
A_{\mu_{1}} v_{i} \leq a_{0} v_{i}+R_{M}^{2 \delta} \varepsilon^{-\delta} .
$$

We will now dominate $A_{\mu_{2}} v_{i}$. Using (6.26), we get

$$
\int_{G} N(g)^{\delta} \mathrm{d} \mu_{2}(g) \leq R_{M}^{-\delta} \int_{G} N(g)^{2 \delta} \mathrm{d} \mu_{M, x}(g) \leq I_{M} R_{M}^{-\delta}=\frac{1-a_{0}}{2} .
$$

By (6.27), this gives

$$
A_{\mu_{2}} v_{i}(x) \leq \frac{1-a_{0}}{2} v_{i}(x) .
$$

Adding (6.28) and (6.29) and summing over $i$, one gets $A_{\mu} v_{M} \leq a_{M} v_{M}+C_{M}$, as required.

\section{BEFORE THE DRIFT}

In this chapter, we begin the proof of Theorem 2.7. We introduce the main notation and we explain the structure of the reduction process.

The main output of this chapter is the fact that, roughly speaking, one can find in $X$ pairs of points close to each other which are generic for $\nu_{b}$ and which are not on the same stable leaf (Corollary 1.11). This is a consequence of the non-degeneracy of the $\nu_{b}$ 's (Proposition 7.8).

We end this chapter by the equivariance properties of the horocyclic flow $\Phi$ and the associated conditional measure map $\sigma$ (Lemmas 7.14 and 7.15).

\subsection{Preliminary reductions.}

In this section, we reduce the proof of Theorem 2.7 to the case where $\nu$ fills $X$, that is, roughly speaking, when the stationary probability measure $\nu$ is not supported by a smaller-dimensional orbit. 
From now on, $G$ is an $\mathcal{S}$-adic Lie group, $\mathfrak{g}$ is its Lie algebra, $\Lambda$ is a discrete subgroup of $G, X=G / \Lambda, \mu$ is an Ad-semisimple probability measure on $G$ and $\nu$ is a $\mu$-ergodic $\mu$-stationary probability measure on $X$. We still let $\Gamma_{\mu}$ be the closed subgroup of $G$ generated by the support of $\mu$ and $H_{\mu} \subset \mathrm{GL}(\mathfrak{g})$ be the Zariski closure of the group $\operatorname{Ad}\left(\Gamma_{\mu}\right)$. We assume that $H_{\mu}=H_{\mu}^{n c}$.

We may assume that $G$ is second countable. Indeed we may always replace $G$ by an open second countable subgroup containing the support of $\mu$, since such a subgroup intersects $\Lambda$ in a lattice.

We also assume that $\left(G, \Gamma_{\mu}\right)$ has good exponentials. Recall from Proposition 5.11 that this is the case as soon as $G$ is a real Lie group or a closed subgroup of some $\operatorname{GL}\left(d, \mathbb{Q}_{\mathcal{S}}\right)$. In particular, if $\mathfrak{l}$ denotes the centralizer of $\Gamma_{\mu}$ in $\mathfrak{g}$ and $L$ the centralizer of $\Gamma_{\mu}$ in $G, \mathfrak{l}$ is the Lie algebra of $L$.

Definition 7.1. We will say that $\nu$ fills $X$ if, for every closed non-open subgroup $G^{\prime}$ of $G$ containing $\Gamma_{\mu}$ and every $x \in X$, one has $\nu\left(G^{\prime} x\right)=0$.

Remark 7.2. There might not exist a smallest open subgroup $G^{\prime} \supset \Gamma_{\mu}$ of $G$ such that $\nu\left(G^{\prime} x\right)=1$. For example, if $G$ is $\operatorname{SL}(2, \mathbb{R}) \times \operatorname{SL}\left(2, \mathbb{Q}_{p}\right), \Lambda$ is the lattice $\operatorname{SL}\left(2, \mathbb{Z}\left[\frac{1}{p}\right]\right)$ in $G, X=G / \Lambda, \mu \in \mathcal{P}(G)$ is a probability measure such that $\Gamma_{\mu}=\operatorname{SL}(2, \mathbb{R})$ and $\nu$ is the $G$-invariant probability measure on $X$, then $\nu$ fills $X$, but for every open subgroup $G^{\prime}$ of $G$, one has $\Gamma_{\mu} \subset G^{\prime}$ and $G^{\prime} \Lambda=G$.

Proposition 7.3. To prove Theorem 2.7, it is enough to prove it when $\nu$ fils $X$.

Proof of Proposition 7.3. Let $G^{\prime}$ be a non-open closed subgroup of $G$ which supports the probability $\mu$ and such that some orbit $X^{\prime}$ of $G^{\prime}$ in $X$ supports the stationary probability measure $\nu$. Choose $G^{\prime}$ to have the smallest possible dimension. This orbit $X^{\prime}$ may not be closed or have finite volume. However, since the injectivity radius of $X^{\prime}$ is bounded below by the injectivity radius of $X$, i.e. since

$$
r_{X^{\prime}}(x) \geq r_{X}(x) \text { for all } x \in X^{\prime},
$$

$\left(G^{\prime}, X^{\prime}, \mu\right)$ inherits exponential $\mu$-unstability of the cusps from $(G, X, \mu)$. By construction, $\nu$ fills $X^{\prime}$. We can then apply Theorem 2.7 to $\left(G^{\prime}, \mu, X^{\prime}, \nu\right)$, and we get that $\nu$ is $\Gamma_{\mu}$-invariant and homogeneous.

\subsection{Construction of $S$.}

In this section we explain how to construct a nice subgroup $S$ of $G$ which preserves $\nu$ and whose normalizer $G^{\prime}$ is open in $G$. Most of the proof of Theorem 2.7 in the following sections will then take place in the quotient group $\underline{G}:=G^{\prime} / S$.

We set $G_{\nu}:=\left\{g \in G \mid g_{*} \nu=\nu\right\}$ for the stabilizer of $\nu$ in $G$,

$$
G_{\nu}^{\prime}:=\bigcap_{g \in \Gamma_{\mu}} g G_{\nu} g^{-1},
$$

and $L_{\nu}:=L \cap G_{\nu}$. The strategy of the proof consists in constructing $\operatorname{Ad}_{\mathfrak{g}}$-unipotent one-parameter subgroups of $G_{\nu}^{\prime}$. We hence let $S_{\max }=\left(G_{\nu}^{\prime}\right)_{u}$ be the closed subgroup of $G_{\nu}^{\prime}$ which is generated by the $\mathrm{Ad}_{\mathfrak{g}}$-unipotent one-parameter subgroups contained in $G_{\nu}^{\prime}$. By construction, the group $S_{\max }$ is normalized by $\Gamma_{\mu}$.

Here comes the second reduction step in the proof of Theorem 2.7

Proposition 7.4. Let $G$ be a weakly regular $\mathcal{S}$-adic Lie group, $\Lambda$ be a discrete subgroup of $G, X=G / \Lambda, \mu$ be an Ad-semisimple probability measure on $G$ such that $\left(G, \Gamma_{\mu}\right)$ has good exponentials, $H_{\mu}=H_{\mu}^{n c}$ and $\int_{G} \log \|\operatorname{Ad} g\| \mathrm{d} \mu(g)<\infty$. 
Let $\nu \in \mathcal{P}(X)$ be a $\mu$-stationary $\mu$-ergodic probability measure that fills $X$. Then there exists a closed subgroup $S$ of $G$ and $x=g \Lambda$ in $X$ such that

(i) The unimodular normalizer $G^{\prime}=N_{1}(S) \subset G$ is open, contains $\Gamma_{\mu}$ and $\nu\left(G^{\prime} x\right)=1$.

(ii) The group $S$ is semiconnected and $S_{\max } \subset S \subset G_{\nu}$.

(iii) The group $S \cap g \Lambda g^{-1}$ is a lattice in $S$ and $S=\overline{S_{\max }\left(S \cap g \Lambda g^{-1}\right)}$.

Proof of Proposition 7.4. To construct the subgroup $S$, we use Ratner's Theorem 5.15. Let $\mathcal{E}$ be, as in (5.6), the set of Ratner's probability measures on $X$. Since the group $S_{\text {max }}$ is generated by $\mathrm{Ad}_{\mathfrak{g}}$-unipotent one-parameter subgroups, one can write the decomposition of $\nu$ into $S_{\text {max }}$-ergodic components as

$$
\nu=\int_{X} \alpha_{x} \mathrm{~d} \nu(x)
$$

where the map $x \mapsto \alpha_{x} \in \mathcal{E}$ is Borel and constant on $S_{\max }$-orbits. Since $\Gamma_{\mu}$ normalizes $S_{\max }$ and since this ergodic decomposition is unique, for every $g \in \Gamma_{\mu}$, for $\nu$-almost every $x \in X$, one has $\alpha_{g x}=g_{*} \alpha_{x}$.

Hence the probability measure $\eta \in \mathcal{P}(\mathcal{E})$, which is the image of $\nu$ by the map $x \mapsto \alpha_{x}$, is also $\mu$-stationary and $\mu$-ergodic. Since $G \backslash \mathcal{E}$ is countable, $\eta$ is supported by a $G$-orbit $G \alpha_{0} \simeq G / G_{\alpha_{0}}$. As $G_{\alpha_{0}}$ admits a lattice, it is unimodular. By Proposition 5.19, we can choose $\alpha_{0}$ in such a way that there exists a semi-connected component $S=S_{\alpha_{0}}$ of the stabilizer $G_{\alpha_{0}}$ such that the group $G^{\prime}:=N_{1}(S)$ contains $\Gamma_{\mu}$ and the stationary measure $\eta$ is supported by $G^{\prime} \alpha_{0}$. In other words, for $\nu$-almost every $x$ in $X$, we have $\eta\left(G^{\prime} \alpha_{x}\right)=1$. Hence, as $G^{\prime}$ contains $G_{\alpha_{0}}, \nu\left(G^{\prime} x\right)=1$ (one can prove that the orbit $G^{\prime} x$ is closed in $X$ - see [15. Th. 3.4] when $G$ is a real Lie group - but we will not use this fact). Since $\nu$ fills $X$, the group $G^{\prime}$ is open in $G$. By construction, since $\alpha_{0}$ is a Ratner probability measure, for $\nu$-almost any $x=g \Lambda$ in $X, S \cap g \Lambda g^{-1}$ is a lattice in $S$ and $S=\overline{S_{\max }\left(S \cap g \Lambda g^{-1}\right)}$.

The case where $S_{\max }=\{1\}$ is the crucial one in Theorem 2.7 We state it as a corollary of Theorem 2.7 .

Corollary 7.5. Let $G$ be a weakly regular $\mathcal{S}$-adic Lie group, $\Lambda$ be a discrete subgroup of $G, X=G / \Lambda, \mu$ be an Ad-semisimple compactly supported probability measure on $G$ such that $H_{\mu}=H_{\mu}^{n c}$ and $L$ be the centralizer of $\Gamma_{\mu}$ in $G$. We assume that the cusps of $X$ are exponentially $\mu$-unstable. Let $\nu \in \mathcal{P}(X)$ be a $\mu$-stationary $\mu$-ergodic probability measure. If one has $S_{\max }=\{1\}$, then $\nu$ is $\Gamma_{\mu}$-invariant, homogenous and supported by a finite union of L-orbits.

We will see in Lemma 7.6 that the conclusion of Theorem 2.7 implies that $\nu$ is homogeneous under the group $\Gamma_{\mu} L_{\nu} S$.

When proving Theorem 2.7 we may assume that $G^{\prime}=G$, i.e. that $S$ is normal in $G$. Since $\Lambda \cap S$ is a lattice in $S$, the group $\underline{\Lambda}:=\Lambda S / S$ is a lattice in $\underline{G}:=G / S$. Let $\underline{X}$ be the quotient space $\underline{X}:=\underline{G} / \underline{\Lambda}$ and $\mu$ and $\underline{\nu}$ be the probability measures on $\underline{G}$ and $\underline{X}$ which are the images of $\mu$ and $\bar{\nu}$ by the natural maps $G \rightarrow \underline{G}$ and

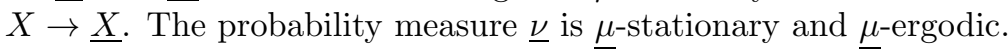

When $G$ is a real Lie group, Theorem 2.7 is a direct consequence of Corollary 7.5 applied to $(\underline{G}, \underline{\Lambda}, \underline{X}, \underline{\mu}, \underline{\nu})$.

When $G$ is a weakly regular $\mathcal{S}$-adic Lie group, the quotient group $\underline{G}$ might not be weakly regular. This is why we have to be a little bit more careful. By Proposition $[5.11$ ) $),(\underline{G}, \underline{\Gamma})$ has good exponentials. Hence we will be able to work out the main 
part of the proof of Theorem 2.7 in this quotient. It is only at the very end of the proof in section 8.3 that we will lift the information obtained so far to the weakly regular group $G$.

The proof of Theorem 2.7 will last up to the end of this paper. Using Proposition 7.3. we can assume that $\nu$ fills $X$. Using Lemma 7.6 below, we can assume that for all $x \in X$ one has $\nu(L S x)=0$. Our aim will then be to get a contradiction by constructing $\Gamma_{\mu}$-unstable one-parameter subgroups of $G$ preserving $\nu$.

\subsection{Stationary measures on orbits of the centralizer.}

In this section we study those stationary probability measures $\nu$ on $X$ for which some $L$-orbit satisfies $\nu(L x)>0$.

We point out that this group $L$, which is the centralizer of $\Gamma_{\mu}$ in $G$, is not assumed to be compact; hence its dynamics on $X$ might be intricate.

Lemma 7.6. Let $G$ be a locally compact second countable group, $\Lambda$ be a discrete subgroup of $G, X=G / \Lambda, \mu$ be a probability measure on $G$ and $L$ be the centralizer of $\Gamma_{\mu}$ in $G$. Let $\nu \in \mathcal{P}(X)$ be a $\mu$-stationary $\mu$-ergodic probability measure on $X$. If one has $\nu(L x)>0$ for some $x$ in $X$, then $\nu$ is $\Gamma_{\mu}$-invariant and homogenous.

More precisely, $\nu$ is homogeneous under the closed group $\Gamma_{\mu} L_{0}$, where $L_{0}$ is any open subgroup of the stabilizer $L_{\nu}$ of $\nu$ in $L$.

Proof. By Lemma 3.7. for $\nu$-almost any $x$ in $X$, one has $\nu(L x)=\nu\left(L_{\nu} x\right)$. By assumption, there exists a point $x$ in $X$ with $\nu(L x)>0$. As $L_{0}$ is open in $L_{\nu}$, there exists a point $x$ such that $\nu\left(L_{0} x\right)>0$. Let $Y$ be the set of those $x$ in $X$ such that $\nu\left(L_{0} x\right)$ takes its maximal value $M$. Since every $L_{0}$-orbit of finite volume in $X$ is closed and since $Y$ is a finite union of $L_{0}$-orbits, this set $Y$ is closed. As $\nu$ is $\mu$-stationary, for any $x$ in $X$ we have $\nu\left(L_{0} x\right)=\int_{G} \nu\left(L_{0} g^{-1} x\right) \mathrm{d} \mu(g)$ so that, if $x$ belongs to $Y$, by the maximum principle, $\nu\left(L_{0} g^{-1} x\right)=M$ for $\mu$-almost any $g$ in $G$. As $Y$ is closed, $Y$ is invariant by the semigroup generated by the support of $\check{\mu}$. As $Y$ is a union of finitely many $L_{0}$-orbits, $Y$ is also invariant by the group $\Gamma_{\check{\mu}}=\Gamma_{\mu}$. As $\nu$ is $\mu$-ergodic, $\nu$ is then supported by $Y, \nu$ gives equal mass to the $L_{0}$-orbits and $\Gamma_{\mu}$ transitively permutes these $L_{0}$-orbits. Hence $\Gamma_{\mu}$ also permutes the $L_{0}$-invariant probability measures supported by these $L_{0}$-orbits. Hence $\nu$ is invariant under the group $\Gamma_{\mu} L_{0}$.

This group $\Gamma_{\mu} L_{0}$ is closed. Indeed, its closure $G^{\prime}:=\overline{\Gamma_{\mu} L_{0}}$ acts transitively on $Y$ with discrete stabilizers and the $L_{0}$-orbits in $Y$ are open. Hence the group $L_{0}$ is open in $G^{\prime}$ and the group $\Gamma_{\mu} L_{0}$ is also open in $G^{\prime}$. This proves that $\Gamma_{\mu} L_{0}$ is closed.

Remark 7.7. The group $L_{0}$ may happen to be non-trivial. For example, let $G=$ $G_{1} \times G_{2}$, where $G_{1}=\operatorname{SL}(2, \mathbb{R})$ and $G_{2}$ is compact and where $\Lambda \subset G$ is a lattice whose projection on $G_{2}$ is dense. We let $\Gamma_{\mu}$ be the projection of $\Lambda$ on $G_{1}$, which is a lattice in $G_{1}$. Then the orbit closure of the base point of $G / \Lambda$ under $\Gamma_{\mu}$ is its orbit under $G_{2}$ and $\Gamma_{\mu}$ acts on it through a morphism with dense image in $G_{2}$. In particular, the $G_{2}$-invariant probability measure on this orbit is $\mu$-stationary and concentrated on an orbit of the centralizer of $\Gamma_{\mu}$.

\subsection{Where we use the positive $\mu$-unstability of the diagonal.}

The aim of this section is to show that if $\nu$ does not give mass to the $L$-orbits, then the limit probability measures $\nu_{b}$ do. 
We will use freely the dynamical systems $(B, \mathcal{B}, \beta, T),\left(B^{X}, \mathcal{B}^{X}, \beta^{X}, T^{X}\right)$, $\left(B^{\theta}, \mathcal{B}^{\theta}, \beta^{\theta}, T^{\theta}\right),\left(B^{\theta, X}, \mathcal{B}^{\theta, X}, \beta^{\theta, X}, T^{\theta, X}\right)$ introduced in sections 3.23 .3 and 3.4 for those values of $G$ and $\mu$ and where, for $b$ in $B, \rho(b)$ is the automorphism $x \mapsto b_{0}^{-1} x$ of $X, \nu_{b}$ is the limit probability measure of $\nu$ associated to $b$ and $\theta(b)=\sigma\left(b_{0}, \xi_{T b}\right) \in Z_{\mu}$ is as in (4.1). We may apply the results of section 4 since all the representations of $\Gamma_{\mu}$ that we will consider are restrictions of representations of the semisimple linear group $H_{\mu}$.

Proposition 7.8 (Non-degeneracy of the $\nu_{b}$ 's). Let $G$ be an $\mathcal{S}$-adic Lie group, $\Lambda$ be a discrete subgroup of $G, X=G / \Lambda, \mu$ be an Ad-semisimple probability measure on $G$ such that $\left(G, \Gamma_{\mu}\right)$ has good exponentials, $H_{\mu}=H_{\mu}^{n c}$, the cusps of $X$ are exponentially $\mu$-unstable, and $\mu$ admits finite exponential moments in $\mathfrak{g}$. Let $\nu$ be a $\mu$-stationary $\mu$-ergodic probability measure on $X$ and $L$ be the centralizer of $\Gamma_{\mu}$ in $G$. Suppose, for every $x$ in $X$, one has $\nu(L x)=0$. Then, for $\beta^{X}$-almost every $(b, x)$ in $B^{X}$, one has $\nu_{b}\left(L W_{b}(x)\right)=0$.

Here, we have equipped $G / \Lambda$ with the distance coming from a right-invariant distance on $G$. We recall that $W_{b}(x)$ has been defined in (6.22). We have explained in Lemma 7.6 what happens when some $L$-orbit satisfies $\nu(L x)>0$.

We will need the following lemma. Let $L_{0}$ be an open subgroup of the stabilizer $L_{\nu}$ of $\nu$ in $L$ generated by some compact standard neighborhood of $e$.

Lemma 7.9. Let $G$ be an $\mathcal{S}$-adic Lie group, $\Lambda$ be a discrete subgroup of $G, X=$ $G / \Lambda, \mu$ be a probability measure on $G, L$ be the centralizer of $\Gamma_{\mu}$ in $G$ and $\nu$ be a $\mu$-stationary $\mu$-ergodic probability measure on $X$. Assume $\nu$ fills $X$ and, for $\beta^{X}$ almost every $(b, x) \in B^{X}$, one has $\nu_{b}\left(L_{0} x\right)>0$. Then there exists a cocompact subgroup $\Lambda_{0} \subset L_{0}$ which fixes every point of the support of $\nu$ and whose centralizer $G^{\prime}$ is open in $G$ and contains $\Gamma_{\mu}$.

The conclusion means that the group $G^{\prime}$ acts on the support of $\nu$ through the group $G^{\prime} /\left(G^{\prime} \cap \Lambda_{0}\right)$.

Remark 7.10. We have introduced this open subgroup $L_{0}$ of $L_{\nu}$ because we do not know whether the lattices in $L_{\nu}$ are finitely generated. Since any lattice in a connected real Lie group is finitely generated [24, 6.18], any lattice in $L_{0}$ is also finitely generated.

Proof of Lemma 7.9. Since the probability measure $\nu$ on $X$ is $L_{0}$-invariant and the actions of $\Gamma_{\mu}$ and $L$ commute, for $\beta$-almost every $b \in B$, the limit probability measure $\nu_{b}$ is also $L_{0}$-invariant.

By assumption, for $\beta$-almost every $b \in B$, the limit probability $\nu_{b}$ is supported by countably many $L_{0}$-orbits. Hence, for $\nu_{b}$-almost every $x \in X$, the stabilizer $\Lambda_{0}(x)$ of $x$ in $L_{0}$ is a lattice in $L_{0}$. Since $\nu$ is the average of the $\nu_{b}$ 's, for $\nu$-almost every $x$ in $X, \Lambda_{0}(x)$ is also a lattice in $L_{0}$.

The map $x \mapsto \Lambda_{0}(x)$ takes values in the set of lattices of $L_{0}$ and is measurable for the Borel structure induced by the Chabauty topology. This Borel structure is standard. Since the actions of $\Gamma_{\mu}$ and $L$ commute, this map is constant on the $\Gamma_{\mu}$-orbits. Since $\nu$ is $\mu$-ergodic, there exists a lattice $\Lambda_{0} \subset L_{0}$ such that, for $\nu$ almost every $x$ in $X, \Lambda_{0}(x)=\Lambda_{0}$. Hence the support of $\nu$ is contained in the set $X^{\Lambda_{0}}$ of fixed points of $\Lambda_{0}$ in $X$. The centralizer $G^{\prime}$ of $\Lambda_{0}$ contains the group $\Gamma_{\mu}$. Since the lattice $\Lambda_{0}$ is finitely generated, according to Lemma [5.16, the set $X^{\Lambda_{0}}$ is a countable union of $G^{\prime}$-orbits. By $\mu$-ergodicity of $\nu$, the support of $\nu$ is included in 
a $G^{\prime}$-orbit $G^{\prime} x$. Since $\nu$ fills $X$, the group $G^{\prime}$ is open in $G$. The group $\Lambda_{0}^{\prime}=\Lambda_{0} \cap G^{\prime}$ is also a lattice in the group $L_{0}^{\prime}=L_{0} \cap G^{\prime}$. Since $\Lambda_{0}^{\prime}$ is central in $L_{0}^{\prime}$, this lattice $\Lambda_{0}^{\prime}$ is cocompact in $L_{0}^{\prime}$. Since $L_{0}$ contains a cocompact connected subgroup, $L_{0}^{\prime}$ has finite index in $L_{0}$. Hence the lattice $\Lambda_{0}$ is also cocompact in $L_{0}$.

Proof of Proposition 7.8. As in Proposition 7.3, we may assume that $\nu$ fills $X$. By Proposition 6.18, it is enough to check that

$$
\text { for } \beta^{X} \text {-almost every }(b, x) \text { in } B^{X} \text {, one has } \nu_{b}(L x)=0 \text {. }
$$

We will argue by contradiction. By ergodicity of $\nu$, we may assume that, for $\beta^{X_{-}}$ almost every $(b, x) \in B^{X}$, one has $\nu_{b}(L x)>0$. We want to check that, for $\nu$-almost every $x \in X$, one has $\nu(L x)>0$.

Again by Lemma 3.7, there exists an $L_{\nu}$-invariant Borel subset $E$ of $X$ such that $\nu(E)=1$ and, for all $l \in L \backslash L_{\nu}$, one has $l E \cap E=\emptyset$. In particular, one has $\nu_{b}(E)=1$ for $\beta$-almost every $b$ in $B$. Hence, for $\beta^{X}$-almost every $(b, x)$ in $B^{X}$, one has $\nu_{b}\left(L_{0} x\right)>0$.

By Lemma 7.9, there exists a cocompact subgroup $\Lambda_{0} \subset L_{0}$ whose centralizer $G^{\prime}$ is open in $G$, contains $\Gamma_{\mu}$ and acts trivially on $\operatorname{Supp}(\nu)$. As $\nu$ is ergodic, $\nu$ is supported by a $G^{\prime}$-orbit and we may assume that $G^{\prime}=G$. Then $\Lambda_{0}$ is a central subgroup of $G$ which acts trivially on $X$. Since the group $L_{0} / \Lambda_{0}$ is compact, we may consider the quotient $X^{\prime}:=L_{0} \backslash X$ which is a locally compact space. The group $\Gamma_{\mu}$ still acts on $X^{\prime}$, and the image $\nu^{\prime}$ of $\nu$ by the natural map $X \rightarrow X^{\prime}$ is still $\mu$-stationary and $\mu$-ergodic and atom-free. We set $\nu_{b}^{\prime}$ for its limit probabilities. To prove (7.1), we have to check that

$$
\text { for } \beta \text {-almost every } b \text { in } B \text {, the probability } \nu_{b}^{\prime} \text { is atom-free. }
$$

Here comes the main argument which is a combination of Corollary 6.26 and Proposition 6.17. Since $\left(G, \Gamma_{\mu}\right)$ has good exponentials and the cusps of $X$ are exponentially $\mu$-unstable, we can apply Corollary 6.26 and prove that the orbit of the diagonal $\left(L_{0} \times L_{0}\right) \Delta_{X}$ is positively $\mu$-unstable in $X \times X$. This means that the diagonal $\Delta_{X^{\prime}}$ is positively $\mu$-unstable in $X^{\prime} \times X^{\prime}$.

Hence, since $\nu^{\prime}$ is atom-free, by Proposition 6.17, for $\beta$-almost every $b$ in $B$, the probability $\nu_{b}^{\prime}$ is also atom-free. This proves (7.2).

\subsection{Narrowing the leaves.}

In this section we apply Proposition 7.8 in order to obtain the starting point of the exponential drift argument in section 8.1 roughly speaking, for any positive measure subset $F$ of $B^{\theta, X}, \beta^{\theta, X}$ almost every point $(b, x)$ of $F$ can be reached by points $\left(b, x^{\prime}\right)$ of $F$ which lie outside the stable leaf of $(b, x)$.

We will use notions and notation of sections 4.2 , 4.3, 5.1 and 5.2

Let $\mathfrak{v}$ be the $\Gamma_{\mu}$-invariant complementary subset of the centralizer $\mathfrak{l}$ of $\Gamma_{\mu}$ in $\mathfrak{g}$. We decompose $\mathfrak{v}$ as a direct sum of irreducible representations $\mathfrak{v}_{i}$ of $H_{\mu}$, where $i$ varies in a finite set $I$. Note that each subspace $\mathfrak{v}_{i}$ is contained in some factor $\mathfrak{g}_{p_{i}}$, $p_{i} \in \mathcal{S}$, and hence is a $\mathbb{Q}_{p_{i}}$-vector space. We choose a $(K, A)$-good norm $\|\cdot\|$ on each of the $\mathfrak{v}_{i}$, and we equip $\mathfrak{v}$ with the norm given, for $v=\left(v_{i}\right)$, by $\|v\|=\max _{i}\left\|v_{i}\right\|$.

For $i$ in $I$, we denote by $\chi_{i}$ the unique morphism $Z_{\mu} \rightarrow \mathbb{R}_{+}^{*}$ whose restriction to $A_{\mu}$ is the modulus of the highest weight of $A$ in $\mathfrak{v}_{i}$ so that, for any $a$ in $A_{\mu}$ and $v$ 
in the highest weight space of $\mathfrak{v}_{i},\|a v\|=\chi_{i}(a)\|v\|$. For $\beta$-almost every $b \in B$, we set

$$
\mathfrak{w}_{i, b}=\left\{v \in \mathfrak{v}_{i} \mid \sup _{n \in \mathbb{N}}\left(\chi_{i}\left(\theta_{n}(b)\right)\left\|b_{n}^{-1} \cdots b_{0}^{-1} v\right\|\right)<\infty\right\}
$$

and $\mathfrak{w}_{b}=\bigoplus_{i \in I} \mathfrak{w}_{i, b}$. This space does not depend on the choice of the decomposition of $\mathfrak{v}$. In particular, it is stable by the adjoint action of $L$. For $c=(b, z)$ in $B^{\theta}$, we set $\mathfrak{w}_{c}=\mathfrak{w}_{b}$ and $\nu_{c}=\nu_{b}$.

The following corollary of Proposition 7.8 extends [4, Corol. 6.15].

Corollary 7.11 (Construction of nearby points). We keep the notation and assumptions of Proposition 7.8. Let $F \subset B^{\theta, X}$ be a $\mathcal{B}^{\theta, X}$-measurable subset such that $\beta^{\theta, X}(F)>0$. Then, for $\beta^{\theta, X}$-almost every $(c, x) \in F$, there exists a sequence $\left(u_{p}\right)$ of elements of $\mathfrak{g} \backslash\left(\mathfrak{l} \oplus \mathfrak{w}_{c}\right)$ converging to 0 such that, for every integer $p \geq 0$, one has $\left(c, \exp \left(u_{p}\right) x\right) \in F$.

Proof. By Proposition 4.4, for any $i$ in $I$ and for $\beta$-almost every $b$ in $B$, one has $\chi_{i}\left(\theta_{n}(b)\right) \underset{n \rightarrow \infty}{\longrightarrow} \infty$. Therefore, for every $v$ in $\mathfrak{w}_{b}$, one has $\left\|b_{n}^{-1} \cdots b_{0}^{-1} v\right\| \underset{n \rightarrow \infty}{\longrightarrow} 0$ and $v$ is $\Gamma_{\mu}$-unstable. Let us choose a distance $d$ on $X$, which comes from a rightinvariant distance on the group $G$. For $\beta$-almost every $b \in B$, all $x \in X$ and all $v \in \mathfrak{w}_{b}$, one has

$$
d\left(b_{n}^{-1} \cdots b_{0}^{-1} \exp (v) x, b_{n}^{-1} \cdots b_{0}^{-1} x\right) \underset{n \rightarrow \infty}{\longrightarrow} 0,
$$

that is, $\exp \left(\mathfrak{w}_{b}\right) x \subset W_{b}(x)$. By Proposition 7.8, for $\beta^{X}$-almost every $(b, x) \in B^{X}$, one has

$$
\nu_{b}\left(L \exp \left(\mathfrak{w}_{b}\right) x\right) \leq \nu_{b}\left(L W_{b}(x)\right)=0 .
$$

Now let $\left(U_{p}\right)$ be a basis of neighborhoods of 0 in $\mathfrak{g}$. For $\beta^{\theta}$-almost every $c$ in $B^{\theta}$, the slice $F_{c}=\{x \in X \mid(c, x) \in F\}$ satisfies $\nu_{c}\left(F_{c}\right)>0$. Therefore, for $\beta^{\theta, X}$-almost every $(c, x)$ in $F$, for all $p \geq 0$, one has

$$
\nu_{c}\left(F_{c} \cap \exp \left(U_{p}\right) x\right)>0 .
$$

As $L$ normalizes $\mathfrak{w}_{c}$, if $p$ is large enough, one has

$$
\exp \left(U_{p} \cap\left(\mathfrak{l} \oplus \mathfrak{w}_{c}\right)\right) \subset L \exp \left(\mathfrak{w}_{c}\right) .
$$

By (7.3), one has $\nu_{c}\left(\exp \left(U_{p} \cap\left(\mathfrak{l} \oplus \mathfrak{w}_{c}\right)\right) x\right)=0$. Therefore, one gets $\nu_{c}\left(F_{c} \cap \exp \left(U_{p} \backslash\right.\right.$ $\left.\left.\left(\mathfrak{l} \oplus \mathfrak{w}_{c}\right) x\right)\right)>0$.

\subsection{Horocyclic flow and conditional measures.}

The aim of this section is to construct a multiparameter flow $\Phi$ on $B^{\theta, X}$ which commutes with the transformation $T^{\theta, X}$. We call it the horocyclic flow since it plays the same role as the horocyclic flow in [4]. We then define the associated conditional measures map $\sigma$. We compare the value of this map $\sigma$ at two points on the same horocyclic orbit, and we show that this map $\sigma$ is $T^{\theta, X}$-invariant.

For $\beta$-almost any $b$ in $B$, we let $\mathfrak{v}_{b}$ and $V_{b}$ be as in Proposition 5.13, with $\mathfrak{g}^{\prime}=\mathfrak{g}$. We set $\mathfrak{v}_{0}=\mathfrak{v}_{\eta_{\mu}}$. By replacing $A$ by a conjugate maximal split torus of $H_{\mu}$, we can assume $\mathfrak{v}_{0}=\mathfrak{v}_{b}$ for some $b$ in $B$ which satisfies all the conclusions of Proposition 5.13. We then set $V_{0}=\exp \left(\mathfrak{v}_{0}\right)$. 
Definition 7.12. The horocyclic flow is the action $\Phi$ of $V_{0}$ on $B^{\theta, X}$ given by, for every $v_{0}$ in $\mathfrak{v}_{0}, \beta^{\theta}$-almost every $c=(b, z)$ in $B^{\theta}$ and every $x$ in $X$,

$$
\Phi_{\exp \left(v_{0}\right)}(c, x)=\left(c, \exp \left(v_{0}^{s\left(\xi_{b}\right) z}\right) x\right) .
$$

In this definition, $v_{0}^{s\left(\xi_{b}\right) z}$ denotes the element of $\mathfrak{g}$ which is the image of $v_{0}$ by the action of $s\left(\xi_{b}\right) z \in H_{\mu}$. We emphasize the fact that the flow $\Phi$ might not preserve the measure $\beta^{\theta, X}$.

Lemma 7.13. We assume that $\left(G, \Gamma_{\mu}\right)$ has good exponentials. For all $v_{0} \in \mathfrak{v}_{0}$, one has $\Phi_{\exp \left(v_{0}\right)} \circ T^{\theta, X}=T^{\theta, X} \circ \Phi_{\exp \left(v_{0}\right)}$.

Proof. For $\beta^{\theta, X}$-almost any $(c, x)=(b, z, x)$ in $B^{\theta, X}$, one has

$$
\begin{aligned}
\Phi_{\exp \left(v_{0}\right)} \circ T^{\theta, X}(c, x) & =\left(T b, \theta(b)^{-1} z, \exp \left(v_{0}^{s\left(\xi_{T b}\right) \theta(b)^{-1} z}\right) b_{0}^{-1} x\right) \\
& =\left(T b, \theta(b)^{-1} z, b_{0}^{-1} \exp \left(v_{0}^{s\left(\xi_{b}\right) z}\right) x\right) \\
& =T^{\theta, X} \circ \Phi_{v_{0}}(c, x),
\end{aligned}
$$

in which we used the equality $b_{0} s\left(\xi_{T b}\right)=s\left(\xi_{b}\right) \theta(b)$ which holds for $\beta$-almost every $b \in B$ (see (4.1)).

Let $\mathcal{M}_{1}\left(V_{0}\right)$ be the space of positive Radon measure on $V_{0}$ up to normalization: two positive Radon measures $\sigma_{1}$ and $\sigma_{2}$ are equal up to normalization, and one writes

$$
\sigma_{1} \simeq \sigma_{2} \text { if there exists } C>0 \text { such that } \sigma_{2}=C \sigma_{1} .
$$

We denote by $\sigma: B^{\theta, X} \rightarrow \mathcal{M}_{1}\left(V_{0}\right)$ the map which, to a given $(c, x)$ in $B^{\theta, X}$, assigns the conditional measure of the measure $\beta^{\theta, X}$ along the horocyclic action of $V_{0}$, which is defined, for instance, in [4, Sect. 4.1]. In loc. cit., one defines the conditional measure of a finite measure $\lambda$ along the orbits of a Borel action of a locally compact unimodular group $R$ with discrete stabilizers. Even though the measure $\beta^{\theta, X}$ is infinite, the space $B^{\theta, X}$ is a countable union of $V_{0}$-invariant finite measure subsets so that the definition of $\sigma$ makes sense.

The following lemma compares the values of $\sigma$ at two points on the same horocyclic orbit. For $v$ in $V_{0}$, let $t_{v}$ be the right translation by $v$, that is, $t_{v}\left(v^{\prime}\right)=v^{\prime} v$ for every $v^{\prime} \in V_{0}$.

Lemma 7.14. There exists a Borel subset $E \subset B^{\theta, X}$ with $\beta^{\theta, X}\left(E^{c}\right)=0$ such that, for every $v \in V_{0}$ and $(c, x) \in E$ with $\Phi_{v}(c, x) \in E$, one has

$$
t_{v *} \sigma\left(\Phi_{v}(c, x)\right) \simeq \sigma(c, x) .
$$

Proof. This is Proposition 4.2 of [4].

The following lemma tells us that the map $\sigma$ is $T^{\theta, X}$-invariant:

Lemma 7.15. For $\beta^{\theta, X}$-almost every $(c, x)$ in $B^{\theta, X}$, one has

$$
\sigma\left(T^{\theta, X}(c, x)\right) \simeq \sigma(c, x) .
$$

Proof. Note that, for $\beta^{\theta, X}$-almost every $(b, z, x)$ in $B^{\theta, X}, \sigma(b, z, x)$ is the conditional measure of $\delta_{(b, z)} \otimes \nu_{b}$ along the action of $V_{0}$ on $\{(b, z)\} \times X$. Now the action of $T^{\theta, X}$ on $\{(b, z)\} \times X$ is the map $(b, z, x) \mapsto\left(T b, \theta(b)^{-1} z, b_{0}^{-1} x\right)$ which isomorphically maps the measure $\delta_{(b, z)} \otimes \nu_{b}$ to the measure $\delta_{T^{\theta}(b, z)} \otimes \nu_{T b}$. As, by Lemma 7.13, this map commutes with the action of $V_{0}$, the result follows. 
As in section 3.4, we introduce the tail $\sigma$-algebra $\mathcal{Q}_{\infty}^{\theta, X}$ of the dynamical system $\left(B^{\theta, X}, \mathcal{B}^{\theta, X}, \beta^{\theta, X}, T^{\theta, X}\right)$ :

$$
\mathcal{Q}_{\infty}^{\theta, X}:=\bigcap_{n \geq 1} \mathcal{Q}_{n}^{\theta, X},
$$

where $\mathcal{Q}_{n}^{\theta, X}$ is the sub- $\sigma$-algebra given by (3.9).

Corollary 7.16. The map $\sigma: B^{\theta, X} \rightarrow \mathcal{M}_{1}\left(V_{0}\right)$ is $\mathcal{Q}_{\infty}^{\theta, X}$-measurable.

\section{INVARIANCE OF STATIONARY PROBABILITY MEASURES}

We now end the proof of Theorem 2.7. Here are the main steps.

Using the "drift control" (Proposition 4.21) and the "construction of nearby points" (Corollary 7.11), we explain the exponential drift argument in Proposition 8.1 .

In section 8.2 we apply Proposition 8.1 to the "horocyclic conditional measure" map. This proves some invariance properties for these conditional measures (Proposition 8.5) that we use to disintegrate $\nu$ into probability measures $\nu_{b, x}$ which are invariant under some Ad-unipotent one-parameter subgroups (Corollary 8.4).

Using the equivariance of this disintegration and Proposition 5.19, in section 8.3 we prove that these probability measures $\nu_{b, x}$ are indeed invariant under a common Ad-unipotent one-parameter subgroup.

\subsection{The exponential drift.}

The following proposition is the turning point of our method. In section $\underline{8.3}$ we will apply it to the quotients $(\underline{G}, \underline{\Lambda}, \underline{X}, \underline{\mu}, \underline{\nu})$ and to the map $f=\sigma$ introduced in section 7.2

We keep the notation of the previous chapters. In particular, $G$ is an $\mathcal{S}$-adic Lie group, $\mu$ is an Ad-semisimple probability measure on $G$ with $H_{\mu}=H_{\mu}^{n c}, \nu$ is a $\mu$-stationary $\mu$-ergodic probability measure on $X$ and the symbols $s, \xi, \theta, B^{\theta}, \beta^{\theta}$, $\beta^{\theta, X}, \sigma$, etc., have the same meaning as above.

Proposition 8.1 (Exponential drift). We assume $\mu$ has compact support, the cusps of $X$ are exponentially $\mu$-unstable, $\left(G, \Gamma_{\mu}\right)$ has good exponentials and, for any $x$ in $X$, one has $\nu(L x)=0$. Let $(Y, \mathcal{Y})$ be a standard Borel space, $f: B^{\theta, X} \rightarrow Y$ be a $\mathcal{Q}_{\infty}^{\theta, X}$-measurable map and $E \subset B^{\theta, X}$ be a $\mathcal{B}^{\theta, X}$-measurable subset with $\beta^{\theta, X}\left(E^{c}\right)=$ 0 . Then, for $\beta^{\theta, X}$-almost every $(c, x)$ in $B^{\theta, X}$, for every $\varepsilon>0$, there exist a nonzero element $v \in V_{0}$ such that $\|\log v\| \leq \varepsilon$ and an element $\left(c^{\prime}, x^{\prime}\right)$ of $E$ such that $\Phi_{v}\left(c^{\prime}, x^{\prime}\right)$ also belongs to $E$ and

$$
f\left(\Phi_{v}\left(c^{\prime}, x^{\prime}\right)\right)=f\left(c^{\prime}, x^{\prime}\right)=f(c, x) .
$$

Proof. Since one can cover $Z$ by countably many relatively compact open sets $U$, it is enough to fix such a set $U$ and to prove (8.1) for $\beta^{U, X}$-almost every $(c, x) \in B^{U, X}$. We will then look for a point $\left(c^{\prime}, x^{\prime}\right) \in E \cap B^{U, X}$.

By definition, one may consider $Y$ as a compact metric space whose Borel $\sigma$ algebra is $\mathcal{Y}$. Similarly, we may endow $B^{U}$ with the topology of a compact metric space whose Borel $\sigma$-algebra is equal, up to subsets of measure zero, to $\mathcal{B}^{U}$ and such that the natural projection $B^{U} \rightarrow U$ is continuous. We may also endow $B^{U} \times X$ with the product topology of this topology on $B^{U}$ and the usual one on $X$. 
Let $\alpha>0$ be very small. By Lusin's theorem, there exists a compact $K \subset$ $E \cap B^{U, X}$ such that $\beta^{U, X}(K)>1-\alpha^{2}$ and such that the maps $f$ and $(b, z, x) \mapsto \xi_{b}$ are continuous on $K$.

The proof relies on a cautious study of the function $\mathbb{E}\left(\mathbf{1}_{K} \mid \mathcal{Q}_{\infty}^{U, X}\right)$. This function is bounded above by 1 and its average is bounded below by $1-\alpha^{2}$ because

$$
\int_{B^{U, X}} \mathbb{E}\left(\mathbf{1}_{K} \mid \mathcal{Q}_{n}^{U, X}\right)(c, x) \mathrm{d} \beta^{U, X}(c, x)=\beta^{U, X}(K)>1-\alpha^{2} .
$$

Therefore, by Tchebyshev's inequality, the function $\mathbb{E}\left(\mathbf{1}_{K} \mid \mathcal{Q}_{\infty}^{U, X}\right)$ is bounded below by $1-\alpha$ on a set of measure at least $1-\alpha$. Hence there exists a compact set $K^{\prime} \subset E \cap B^{U, X}$ such that $\beta^{U, X}\left(K^{\prime}\right)>1-\alpha$ and that, for every $(c, x)$ in $K^{\prime}$, one has

$$
\mathbb{E}\left(\mathbf{1}_{K} \mid \mathcal{Q}_{\infty}^{U, X}\right)(c, x)>1-\alpha .
$$

Again by Lusin's theorem, we may also assume that $f$ is continuous on $K^{\prime}$.

Moreover, by the martingale convergence theorem, for $\beta^{U, X}$-almost every $(c, x)$ in $B^{U, X}$, one has

$$
\mathbb{E}\left(\mathbf{1}_{K} \mid \mathcal{Q}_{n}^{U, X}\right)(c, x) \underset{n \rightarrow \infty}{\longrightarrow} \mathbb{E}\left(\mathbf{1}_{K} \mid \mathcal{Q}_{\infty}^{U, X}\right)(c, x) .
$$

By Lemma 3.6 we may also assume that, for every $(c, x) \in K^{\prime}$ and every $n \geq 0$, the left-hand side in this equality (8.2) is given by (3.14):

$$
\mathbb{E}\left(\mathbf{1}_{K} \mid \mathcal{Q}_{n}^{U, X}\right)(c, x)=\mu^{\otimes n}\left(h_{n, c, x}^{-1}(K) \mid Q_{n, c}^{U}\right) .
$$

According to (3.4) and (3.10), for $c=(b, z) \in B^{U}, x \in X$ and $a=\left(a_{0}, \ldots, a_{n-1}\right) \in$ $G^{n}$, the elements $h_{n, c}(a) \in B^{\theta}$ and $h_{n, c, x}(a) \in B^{\theta, X}$ which parametrize the atom of $\mathcal{Q}_{n}^{U, X}$ at $x$ are given by

$$
h_{n, c}(a)=\left(a T^{n} b, \theta_{n}\left(a T^{n} b\right) \theta_{n}(b)^{-1} z\right)
$$

and

$$
h_{n, c, x}(a)=\left(h_{n, c}(a), a_{0} \cdots a_{n-1} b_{n-1}^{-1} \cdots b_{0}^{-1} x\right) .
$$

Besides, since $f$ is $\mathcal{Q}_{\infty}^{U, X}$-measurable, it is $\mathcal{Q}_{n}^{U, X}$-measurable for all $n \geq 0$ and, hence, using Lemma 3.5, we may also assume that, for all $(c, x)$ in $K$, for all $n \geq 0$ and for $\mu^{\otimes n}$-almost every $a$ in $G^{n}$, one has $f\left(h_{n, c, x}(a)\right)=f(c, x)$.

Finally, by Egoroff's theorem, we may also assume that the convergence in (8.2) is uniform on $K^{\prime}$. Therefore there exists $n_{0} \geq 0$ such that for every integer $n \geq n_{0}$, for every $(c, x) \in K^{\prime}$, one has

$$
\mathbb{E}\left(\mathbf{1}_{K} \mid \mathcal{Q}_{n}^{U, X}\right)(c, x) \geq 1-\alpha .
$$

Since the $\beta^{U, X}$-measure of $K^{\prime}$ is at least $1-\alpha$ and $\alpha$ is arbitrarily small, it is enough to prove (8.1) for $\beta^{U, X}$-almost every $(c, x)$ of $K^{\prime}$.

Corollary 7.11 allows us to assume that, for these points $(c, x)$ of $K^{\prime}$, there exists a sequence $\left(u_{p}\right)$ of elements of $\mathfrak{g} \backslash\left(\mathfrak{l} \oplus \mathfrak{w}_{c}\right)$ converging to 0 and such that the points $\left(c, y_{p}\right):=\left(c, \exp \left(u_{p}\right) x\right)$ also belong to $K^{\prime}$.

We apply (8.3) and (8.4) to both points $(c, x)$ and $\left(c, y_{p}\right)$. For $n \geq n_{0}$, we then get

$$
\mu^{\otimes n}\left(h_{n, c, x}^{-1}(K) \mid Q_{n, c}^{U}\right) \geq 1-\alpha
$$

and

$$
\mu^{\otimes n}\left(h_{n, c, y_{p}}^{-1}(K) \mid Q_{n, c}^{U}\right) \geq 1-\alpha .
$$


The remaining part of the proof is motivated by the following strategy. By construction, when $y=\exp (u) x$ with $u \in \mathfrak{g}$, the parametrizations of the two fibers of $\left(T^{U, X}\right)^{n}$ containing $(c, x)$ and $(c, y)$ are related by a drift that can easily be computed: if $\left(c^{\prime}, x^{\prime}\right)=h_{n, c, x}(a)$ and $\left(c^{\prime}, y^{\prime}\right)=h_{n, c, y}(a)$, for the same $a \in G^{n}$, one has

where the drift is given by

$$
y^{\prime}=\exp \left(F_{n, c}(a) u\right) x^{\prime}
$$

$$
F_{n, c}(a) u=a_{0} \cdots a_{n-1} b_{n-1}^{-1} \cdots b_{0}^{-1}(u)
$$

as soon as this element is small enough to belong to the logarithm of a $\left(G, \Gamma_{\mu}\right)$ good standard open subset, which will be ensured for most of the relevant $a$ by the computations below. We have just seen that, for the parametrization of the two fibers of $\left(T^{U, X}\right)^{n}$ containing the points $(c, x)$ and $\left(c, y_{p}\right)$, a large proportion of the parameters $a \in G^{n}$ corresponds to points $\left(c^{\prime}, x_{p}^{\prime}\right)$ and $\left(c^{\prime}, y_{p}^{\prime}\right)$ which both belong to $K$. We will now adapt the time $n=n_{p}$ to the sequence $u_{p}$ in such a way that we will control both the norm and the direction of the drift between these two points. This will be possible, thanks to the following lemma that we will derive from the law of the angles through Proposition 4.21.

Lemma 8.2. For all $\alpha>0$ and $\eta>0$, there exists $r_{0}>1$ such that, for $\beta^{U}$-almost every $c \in B^{U}$, for $n$ large enough, one has, for any $i$ in $I$ and any $u_{i}$ in $\mathfrak{v}_{i} \backslash 0$,

$$
\mu^{\otimes n}\left(\left\{a \in G^{n} \mid \frac{1}{r_{0}} \leq \frac{\left\|F_{n, c}(a) u_{i}\right\|}{\chi_{i}\left(\theta_{n}(b)\right)\left\|b_{n-1}^{-1} \cdots b_{0}^{-1} u_{i}\right\|} \leq r_{0}\right\} \mid Q_{n, c}^{U}\right) \geq 1-\alpha
$$

and

$$
\mu^{\otimes n}\left(\left\{a \in G^{n} \mid d\left(\mathbb{R} F_{n, c}(a) u_{i}, \mathbb{P}\left(\mathfrak{v}_{i, a T^{n} b}\right)\right) \leq \eta\right\} \mid Q_{n, c}^{U}\right) \geq 1-\alpha .
$$

We recall that $Q_{n, c}^{U}$ has been defined in (3.5).

Proof. As

$$
F_{n, c}(a) u_{i}=a_{0} \cdots a_{n-1}\left(b_{n-1} \cdots b_{0} u_{i}\right) \text { and } V_{i, a T^{n} b}=a_{0} \cdots a_{n-1} V_{i, T^{n} b}
$$

by (3.8), (8.7) and (8.8) follow directly from Proposition 4.21, applied to the vector $v_{i}=b_{n-1} \cdots b_{0} u_{i}$.

We can now end the proof of Proposition 8.1. We adjust the parameter $n=n_{p}$ in the following way. Since the probability measure $\mu$ on $G$ has compact support and since the section $s$ introduced in section 4.2 has bounded image, there exists a constant $C_{0}>0$ such that, for $\beta$-almost every $b \in B$, for any $i$, any vector $u_{i}$ in $V_{i} \backslash 0$ and any $n \in \mathbb{N}$, one has

$$
\frac{\chi_{i}\left(\theta_{n+1}(b)\right)\left\|b_{n}^{-1} \cdots b_{0}^{-1} u_{i}\right\|}{\chi_{i}\left(\theta_{n}(b)\right)\left\|b_{n-1}^{-1} \cdots b_{0}^{-1} u_{i}\right\|} \leq C_{0} .
$$

Set $M_{0}:=\max _{z \in U, \xi \in \mathcal{P}_{\mu}}\left\|\operatorname{Ad}(s(\xi) z)^{-1}\right\|$. We decompose the vectors $u_{p}$ as a sum

$$
u_{p}=u_{p, \mathfrak{l}}+\sum_{i} u_{p, i}
$$

with $u_{p, \mathfrak{l}} \in \mathfrak{l}$ and $u_{p, i} \in \mathfrak{v}_{i}, i \in I$. Since the elements $u_{p}$ do not belong to $\mathfrak{l} \oplus w_{c}$, the sequence $n \mapsto \max _{i} \chi_{i}\left(\theta_{n}(b)\right)\left\|b_{n}^{-1} \cdots b_{0}^{-1} u_{p, i}\right\|$ is not bounded. For $p$ large enough, there exists an integer $n_{p}$ such that

$$
\frac{\varepsilon}{C_{0} r_{0} M_{0}} \leq \max _{i} \chi_{i}\left(\theta_{n_{p}}(b)\right)\left\|b_{n_{p}-1}^{-1} \cdots b_{0}^{-1} u_{p, i}\right\| \leq \frac{\varepsilon}{r_{0} M_{0}} .
$$


Hence as soon as $\alpha<\frac{1}{2+2 \operatorname{card}(I)}$, there exist an element $a=a_{p}$ in $G^{n_{p}}$ which is simultaneously and for all $i \in I$ for which $u_{p, i} \neq 0$, in the sets described in (8.5), (8.6), (8.7) and (8.8) with $n=n_{p}, u_{i}=u_{p, i}$ and $\eta=\eta_{p} \rightarrow 0$. One then has

$$
f\left(h_{n_{p}, c, x}\left(a_{p}\right)\right)=f(c, x) \text { and } f\left(h_{n_{p}, c, y_{p}}\left(a_{p}\right)\right)=f\left(c_{p}, y_{p}\right) .
$$

After extracting a subsequence:

(1) the sequence $\left(c_{p}^{\prime}, x_{p}^{\prime}\right):=h_{n_{p}, c, x}\left(a_{p}\right)$ has a limit $\left(c^{\prime}, x^{\prime}\right)$ in $K$,

(2) the sequence $\left(c_{p}^{\prime}, y_{p}^{\prime}\right):=h_{n_{p}, c, y_{p}}\left(a_{p}\right)$ has a limit $\left(c^{\prime}, y^{\prime}\right)$ in $K$ and

(3) the limit of the drift $w=\lim _{p \rightarrow \infty} F_{n_{p}, c}\left(a_{p}\right) u_{p}$ exists, is non-zero, with norm bounded by $\frac{\varepsilon}{M_{0}}$ and belongs to $\mathfrak{v}_{c^{\prime}}$.

As a consequence, by passing to the limit in (8.9), since all the sequences involved there take their values in $K$ or $K^{\prime}$ and since $f$ is continuous on these sets, one has

$$
\begin{aligned}
f\left(c^{\prime}, x^{\prime}\right)=\lim _{p \rightarrow \infty} f\left(c_{p}^{\prime}, x_{p}^{\prime}\right) & =\lim _{p \rightarrow \infty} f(c, x)=f(c, x), \\
f\left(c^{\prime}, y^{\prime}\right)=\lim _{p \rightarrow \infty} f\left(c_{p}^{\prime}, y_{p}^{\prime}\right) & =\lim _{p \rightarrow \infty} f\left(c, y_{p}\right)=f(c, x) \text { and } \\
y^{\prime} & =\exp (w) x^{\prime} .
\end{aligned}
$$

Moreover, writing $c^{\prime}=\left(b^{\prime}, z^{\prime}\right)$ and denoting by $v \in \mathfrak{v}_{0}$ the non-zero element such that $w=\exp \left(s\left(\xi_{b^{\prime}}\right) z^{\prime}\right) v$, one has

$$
\|v\| \leq \varepsilon \text { and }\left(c^{\prime}, y^{\prime}\right)=\Phi_{\exp (v)}\left(c^{\prime}, x^{\prime}\right) .
$$

This ends the proof of Proposition 8.1 .

\subsection{The stabilizer of conditional measures.}

In this section, we apply the drift argument to the horocyclic conditional measures map and we express this application in terms which do not involve the extension $B^{\theta}$. We disintegrate the limit measures $\nu_{b}$ according to the values of the stabilizer $V_{b, x}$ in $V_{b}$ of the horocyclic conditionals. Using the Poincaré recurrence theorem thrice, we show that $V_{b, x}$ is a non-trivial Ad-unipotent subgroup of $V_{b}$ which leaves invariant the probability measure $\nu_{b, x}$.

For $\beta^{X}$-almost every $(b, x) \in B^{X}$, we denote by $\sigma_{b, x} \in \mathcal{M}_{1}\left(V_{b}\right)$ the conditional measure of $\nu_{b}$ at $x$ for the action of $V_{b}$ on $X$. By abuse of notation, we also denote by $\sigma_{b, x}$ any element of this class modulo normalization and we let $V_{b, x}$ be the stabilizer of $\sigma_{b, x}$ in $\mathcal{M}_{1}\left(V_{b}\right)$, that is, the group of those $v$ in $V_{b}$ such that $t_{v *} \sigma_{b, x} \simeq \sigma_{b, x}$. We let $\mathfrak{v}_{b, x}$ be the Lie algebra of $V_{b, x}$.

As, for $\beta$-almost any $b$ in $B$, the action of $b_{0}^{-1}$ induces an isomorphism between the measure spaces $\left(X, \mathcal{X}, \nu_{b}\right)$ and $\left(X, \mathcal{X}, \nu_{T b}\right)$, and as $V_{T b}=b_{0}^{-1} V_{b} b_{0}$, one has, for $\nu_{b}$-almost any $x$ in $X$,

$$
V_{T^{X}(b, x)}=b_{0}^{-1} V_{b, x} b_{0} \quad \text { and } \quad \sigma_{T^{X}(b, x)} \simeq\left(\operatorname{Ad} b_{0}^{-1}\right)_{*} \sigma_{b, x} .
$$

Lemma 8.3. Assume $\left(G, \Gamma_{\mu}\right)$ has good exponentials. For $\beta^{X}$-almost any $(b, x)$ in $B^{X}$, one has $V_{b, x}=\exp \left(\mathfrak{v}_{b, x}\right)$ and, for any $v$ in $V_{b, x}, t_{v *} \sigma_{b, x}=\sigma_{b, x}$.

Proof. The proof relies on successive applications of Poincaré's recurrence theorem.

First let us prove that, for $\beta^{X}$-almost every $(b, x)$ in $B^{X}$, one has $\exp \left(\mathfrak{v}_{b, x}\right) \subset$ $V_{b, x}$. Set

$$
\varphi(b, x)=\inf \left\{\|v\| \mid v \in \mathfrak{v}_{b, x}, \exp (v) \notin V_{b, x}\right\} \in[0, \infty]
$$


As $\mathfrak{v}_{b, x}$ is the Lie algebra of $V_{b, x}$, one has $\varphi>0$ almost everywhere. Besides, by the equivariance property, for any $n$ in $\mathbb{N}$, one has

$$
\varphi\left(\left(T^{X}\right)^{n}(b, x)\right) \leq\left\|\left.\left(b_{0} \cdots b_{n-1}\right)^{-1}\right|_{\mathfrak{v}_{b}}\right\| \varphi(b, x) .
$$

Hence, by Proposition [5.13, if $\varphi(b, x)<\infty, \varphi\left(\left(T^{X}\right)^{n}(b, x)\right) \underset{n \rightarrow \infty}{\longrightarrow} 0$ and, by Poincaré's recurrence theorem, $\varphi=\infty$ almost everywhere; that is, $\exp \left(\mathfrak{v}_{b, x}\right) \subset V_{b, x}$ for $\beta^{X}$-almost every $(b, x)$ in $B^{X}$.

Now let us prove that, for $\beta^{X}$-almost every $(b, x)$ in $B^{X}$, one has $V_{b, x} \subset \exp \left(\mathfrak{v}_{b, x}\right)$. Set

$$
\psi(b, x)=\inf \left\{\|v\| \mid v \in \mathfrak{v}_{b} \backslash \mathfrak{v}_{b, x}, \exp (v) \in V_{b, x}\right\} \in(0, \infty] .
$$

Again, if $\psi(b, x)<\infty$, one has $\psi\left(\left(T^{X}\right)^{n}(b, x)\right) \underset{n \rightarrow \infty}{\longrightarrow} 0$. Hence, by Poincaré's recurrence theorem, $\psi=\infty$ almost everywhere and, for $\beta^{X}$-almost any $(b, x)$ in $B^{X}, V_{b, x}=\exp \left(\mathfrak{v}_{b, x}\right)$.

Lastly, set, for $\beta^{X}$-almost any $(b, x)$ in $B^{X}, \alpha_{b, x}: V_{b, x} \rightarrow(0, \infty)$ to be the multiplicative group morphism such that, for any $v$ in $V_{b, x}$, one has $t_{v *} \sigma_{b, x}=$ $\alpha_{b, x}(v) \sigma_{b, x}$. To finish the proof, we shall prove that $\alpha=1$ almost everywhere. As $V_{b, x}=\exp \left(\mathfrak{v}_{b, x}\right)$, there exists a unique homomorphism of real Lie algebras $a_{b, x}:\left(\mathfrak{v}_{b, x}\right)_{\infty} \rightarrow \mathbb{R}$ such that, for any $v$ in $\mathfrak{v}_{b, x}$, one has $\alpha_{b, x}(\exp (v))=e^{a_{b, x}\left(v_{\infty}\right)}$. By the equivariance property, one has, for $\beta^{X}$-almost all $(b, x)$ in $B^{X}$, for any $n$ in $\mathbb{N}$,

$$
a_{b, x}=a_{\left(T^{X}\right)^{n}(b, x)} \circ\left(b_{0} \cdots b_{n-1}\right)^{-1} .
$$

For any $M>0$, let

$$
A_{M}=\left\{(b, x) \in B^{X} \mid\left\|a_{b, x}\right\| \leq M\right\} .
$$

By Poincaré's recurrence theorem, for $\beta^{X}$-almost any $(b, x)$ in $A_{M}$, there exists infinitely many $n$ in $\mathbb{N}$ with $\left(T^{X}\right)^{n}(b, x) \in A_{M}$. By (8.10), we get $a=0$, almost everywhere on $A_{M}$, which should be proved.

For $\beta$-almost any $b$ in $B$, we disintegrate the probability measure $\nu_{b}$ along the Borel map $X \rightarrow \operatorname{Gr}(\mathfrak{g}) ; x \mapsto \mathfrak{v}_{b, x}$. This gives us a decomposition

$$
\nu_{b}=\int_{X} \nu_{b, x} \mathrm{~d} \nu_{b}(x)
$$

where, for $\beta^{X}$-almost every $(b, x)$ in $B^{X}$, the probability measure $\nu_{b, x}$ on $X$ is supported by the fiber $\left\{x^{\prime} \in X \mid \mathfrak{v}_{b, x^{\prime}}=\mathfrak{v}_{b, x}\right\}$.

Corollary 8.4. Assume $\left(G, \Gamma_{\mu}\right)$ has good exponentials. For $\beta^{X}$-almost every $(b, x)$ in $B^{X}$, the probability measure $\nu_{b, x}$ is $V_{b, x}$-invariant and one has the equivariance property

$$
\nu_{b, x}=b_{0 *} \nu_{T^{X}}(b, x) .
$$

Proof. As, by Lemma 8.3. $V_{b, x}$ preserves every element of the class $\sigma_{b, x}$, the first statement follows from [4, Prop. 4.3]. The equivariance property follows from those of the maps $b \mapsto \nu_{b}$ and $(b, x) \mapsto \mathfrak{v}_{b, x}$.

Now, by Proposition 8.1 we know that, under suitable assumptions, $\mathfrak{v}_{b, x}$ is non-zero. 
Proposition 8.5. We assume $\mu$ has compact support, the cusps of $X$ are exponentially $\mu$-unstable, $\left(G, \Gamma_{\mu}\right)$ has good exponentials and, for every $x$ in $X$, one has $\nu(L x)=0$. Then, for $\beta^{X}$-almost every $(b, x) \in B^{X}, V_{b, x}$ is a non-trivial subgroup of $V_{b}$.

Proof. Comparing the definitions of $\sigma(c, x)$ in section 7.6 and of $\sigma_{b, x}$ in section 8.2 . one gets, for $\beta^{\theta, X}$-almost every $(c, x) \in B^{\theta, X}$ with $c=(b, z)$,

$$
\log _{*} \sigma_{b, x}=\left(s\left(\xi_{b}\right) z\right)_{*} \log _{*} \sigma(c, x) .
$$

Hence, it suffices to prove that the stabilizer of $\sigma(c, x)$ in $V_{0}$ is non-zero.

By Lemma 7.14, there exists a Borel subset $E$ of $B^{\theta, X}$ such that $\beta^{\theta, X}\left(E^{c}\right)=0$ and that, for every $v \in V_{0}$ and $\left(c^{\prime}, x^{\prime}\right) \in E$ such that $\Phi_{v}\left(c^{\prime}, x^{\prime}\right) \in E$, one has

$$
t_{v *} \sigma\left(\Phi_{v}\left(c^{\prime}, x^{\prime}\right)\right) \simeq \sigma\left(c^{\prime}, x^{\prime}\right) .
$$

By Corollary 7.16, the map $(c, x) \mapsto \sigma(c, x)$ is $\mathcal{Q}_{\infty}^{\theta, X}$-measurable. Proposition 8.1 applied to this set $E$ and to the map $f=\sigma$ gives us, for $\beta^{\theta, X}$-almost every $(c, x)$ in $B^{\theta, X}$, a non-trivial element $v$ in $V_{0}$ and an element $\left(c^{\prime}, x^{\prime}\right)$ in $E$ such that $\Phi_{v}\left(c^{\prime}, x^{\prime}\right)$ also belongs to $E$ and that

$$
\sigma\left(\Phi_{v}\left(c^{\prime}, x^{\prime}\right)\right) \simeq \sigma\left(c^{\prime}, x^{\prime}\right) \simeq \sigma(c, x) .
$$

Applying equality (8.13) to the element $\left(c^{\prime}, x^{\prime}\right)$, one gets the following result:

$$
t_{v *} \sigma(c, x) \simeq t_{v *} \sigma\left(\Phi_{v}\left(c^{\prime}, x^{\prime}\right)\right) \simeq \sigma\left(c^{\prime}, x^{\prime}\right) \simeq \sigma(c, x) .
$$

\subsection{Invariance of $\nu$ under a one-parameter subgroup.}

In this section, we finish the proof of Theorem 2.7 which classifies $\mu$-stationary $\mu$-ergodic probability measures on $X$. To this aim, we construct a $\mu$-stationary probability measure $\eta$ on the set $\mathcal{E}$ of Ratner probability measures by disintegrating the probability measures $\nu_{b, x}$ into $V_{b, x}$-ergodic components and we analyse $\eta$ thanks to Proposition 5.19 .

Proof of Theorem 2.7. We recall the strategy described in sections 7.1, 7.2 and 7.3 that we have been using since then. Using Proposition 7.3 , we can assume that $\nu$ fills $X$. We can then introduce the groups $S_{\max }$ and $S$ from Proposition 7.4 and assume that $S$ is normal in $G$. Using Lemma 7.6, we assume, by contradiction, that for all $x$ in $X$, one has $\nu(L S x)=0$. We want to get a contradiction.

We apply Corollary 8.4 and Proposition 8.5 to the quotients $(\underline{G}, \underline{\Lambda}, \underline{X}, \mu, \underline{\nu})$ introduced in section 7.2 . This is possible since, by Proposition $5.11 \mathrm{k}),(\underline{G}, \underline{\Gamma})$ has good exponentials, and, by Corollary [5.34, the cusps of $\underline{X}$ are exponentially $\mu$-unstable.

By the definition of $S$, the limit probabilities $\nu_{b}$ are $S$-invariant. Lifting in $X$ (8.11) and (8.12), we get a decomposition

$$
\nu_{b}=\int_{X} \nu_{b, x} \mathrm{~d} \nu_{b}(x)
$$

where for $\beta^{X}$-almost every $(b, x)$ in $B^{X}$, one has

$$
\nu_{b, x}=b_{0 *} \nu_{T^{X}}(b, x) .
$$

We choose a $\Gamma_{\mu}$-invariant complementary subspace to $\mathfrak{s}$ so that $\mathfrak{v}$ identifies to a subspace of $\mathfrak{g}$. Hence, we get a map $B^{X} \rightarrow \operatorname{Gr}(\mathfrak{g}) ;(b, x) \mapsto \mathfrak{v}_{b, x}$. For $\beta^{X}$-almost any $(b, x), \mathfrak{v}_{b, x}$ is contained in $N_{\Gamma}(\mathfrak{g})$. We let $V_{b, x}$ be the subgroup of $G$ spanned 
by $\exp \left(\mathfrak{v}_{b, x}\right)$ and $S_{\max }$. Then, for $\beta^{X}$-almost every $(b, x)$ in $B^{X}, S_{\max }$ is a proper subgroup of $V_{b, x}, \nu_{b, x}$ is $V_{b, x}$-invariant and one has

$$
V_{b, x}=b_{0} V_{T^{X}(b, x)} b_{0}^{-1} .
$$

As $V_{b, x}$ is spanned by Ad-unipotent one-parameter subgroups, by Ratner's Theorem 5.15. the decomposition of $\nu_{b, x}$ into $V_{b, x}$-ergodic components can be written, in a simultaneous way, as

$$
\nu_{b, x}=\int_{X} \zeta\left(b, x^{\prime}\right) \mathrm{d} \nu_{b, x}\left(x^{\prime}\right)
$$

where $\zeta: B^{X} \rightarrow \mathcal{E}$ is a $\mathcal{B}^{X}$-measurable map which is $V_{b, x}$-invariant $\nu_{b, x}$-almost everywhere. As, for $\beta^{X}$-almost any $(b, x)$ in $B^{X}, S_{\max }$ is a proper subgroup of $V_{b, x}$, the stabilizer of $\zeta(b, x)$ in $G$ contains Ad-unipotent one-parameter subgroups which are not contained in $S$.

The end of the proof is analoguous to the proof of Proposition 7.4. Uniqueness of the ergodic decomposition and (8.15) and (8.16) imply that, for $\beta^{X}$-almost every $(b, x)$ in $B^{X}$, one has

$$
\zeta(b, x)=\left(b_{0}\right)_{*} \zeta\left(T^{X}(b, x)\right)
$$

Hence the probability measure $\eta:=\zeta_{*} \beta^{X}$ is a $\mu$-stationary $\mu$-ergodic probability measure on $\mathcal{E}$. By (8.14) and (8.17), one has

$$
\nu=\int_{\mathcal{E}} \alpha \mathrm{d} \eta(\alpha) .
$$

By Ratner's Theorem 5.15, there are only countably many $G$-orbits in $\mathcal{E}$. Since the $\mu$-stationary probability measure $\eta$ is $\mu$-ergodic, it is supported by an orbit $G \alpha_{0} \simeq$ $G / G_{\alpha_{0}}$. Since the stabilizer $G_{\alpha_{0}}$ contains a lattice, it is unimodular. According to Proposition 5.19, we can assume that the group $G_{\alpha_{0}}$ contains a semiconnected component $S_{\alpha_{0}}$ such that the unimodular normalizer $G^{\prime}:=N_{1}\left(S_{\alpha_{0}}\right)$ contains $\Gamma_{\mu}$ and $\eta$ is supported by an orbit of $G^{\prime}$. Therefore, by (8.18), the probability $\nu$ is also $S_{\alpha_{0}}$-invariant, being an average of $S_{\alpha_{0}}$-invariant measures. Now, the subgroup $S_{\alpha_{0}, u}$ of $S_{\alpha_{0}}$ generated by the one-parameter Ad-unipotent subgroups of $S_{\alpha_{0}}$ is not contained in $S$. But $S_{\alpha_{0}, u}$ is included in $G_{\nu}$ and is normalized by $\Gamma_{\mu}$. By Proposition 7.4. this group is contained in $S$. This contradiction ends the proof of Theorem 2.7

\section{REFERENCES}

[1] R. Aoun, Application des marches aléatoires à l'étude des sous-groupes des groupes linéaires, PhD thesis (2011).

[2] Y. Benoist, Propriétés asymptotiques des groupes linéaires, Geom. Funct. Anal. 7 (1997), no. 1, 1-47, DOI 10.1007/PL00001613 (French, with English and French summaries). MR:1437472 (98b:22010)

[3] Y. Benoist, J.-F. Quint, Lattices in $\mathcal{S}$-adic Lie groups, preprint (2010).

[4] Yves Benoist and Jean-François Quint, Mesures stationnaires et fermés invariants des espaces homogènes II, C. R. Math. Acad. Sci. Paris 349 (2011), no. 5-6, 341-345, DOI 10.1016/j.crma.2011.01.015 (French, with English and French summaries). MR2783332 (2012b:60017)

[5] Y. Benoist, J.-F. Quint Mesures stationnaires et fermés invariants des espaces homogènes II, CRAS (2010).

[6] Y. Benoist, J.-F. Quint Stationary measures and invariant subsets of homogeneous spaces (III), en preparation.

[7] Yves Benoist and Jean-Francois Quint, Random walks on finite volume homogeneous spaces, Invent. Math. 187 (2012), no. 1, 37-59, DOI 10.1007/s00222-011-0328-5. MR2874934 
[8] Y. Benoist, J.-F. Quint, Lattices in $\mathcal{S}$-adic Lie groups, preprint, 2010.

[9] Y. Benoist, J.-F. Quint, Random walks on semisimple groups.

[10] Y. Benoist, J.-F. Quint, On p-adic Lie groups.

[11] Philippe Bougerol and Jean Lacroix, Products of random matrices with applications to Schrödinger operators, Progress in Probability and Statistics, vol. 8, Birkhäuser Boston Inc., Boston, MA, 1985. MR886674 (88f:60013)

[12] Jean Bourgain, Alex Furman, Elon Lindenstrauss, and Shahar Mozes, Stationary measures and equidistribution for orbits of nonabelian semigroups on the torus, J. Amer. Math. Soc. 24 (2011), no. 1, 231-280, DOI 10.1090/S0894-0347-2010-00674-1. MR2726604 (2011k:37008)

[13] C. Chabauty, Limites d'ensembles et théorie des nombres, Bull. Soc. Math. Fr. 78 (1950), 143-151.

[14] R. V. Chacon and D. S. Ornstein, A general ergodic theorem, Illinois J. Math. 4 (1960), 153-160. MR0110954 (22 \#1822)

[15] S. G. Dani and G. A. Margulis, Limit distributions of orbits of unipotent flows and values of quadratic forms, I. M. Gel'fand Seminar, Adv. Soviet Math., vol. 16, Amer. Math. Soc., Providence, RI, 1993, pp. 91-137. MR1237827 (95b:22024)

[16] J. D. Dixon, M. P. F. du Sautoy, A. Mann, and D. Segal, Analytic pro-p-groups, London Mathematical Society Lecture Note Series, vol. 157, Cambridge University Press, Cambridge, 1991. MR1152800 (94e:20037)

[17] Alex Eskin and Gregory Margulis, Recurrence properties of random walks on finite volume homogeneous manifolds, Random walks and geometry, Walter de Gruyter GmbH \& Co. KG, Berlin, 2004, pp. 431-444. MR2087794 (2005m:22025)

[18] Yves Guivarc'h and Albert Raugi, Propriétés de contraction d'un semi-groupe de matrices inversibles. Coefficients de Liapunoff d'un produit de matrices aléatoires indépendantes, Israel J. Math. 65 (1989), no. 2, 165-196, DOI 10.1007/BF02764859 (French, with English summary). MR998669(91b:22006)

[19] Jean-Romain Heu, Dynamical properties of groups of automorphisms on Heisenberg nilmanifolds, Geom. Dedicata 145 (2010), 89-101, DOI 10.1007/s10711-009-9407-9. MR2600947 (2011d:37043)

[20] Gregory Margulis, Problems and conjectures in rigidity theory, Mathematics: frontiers and perspectives, Amer. Math. Soc., Providence, RI, 2000, pp. 161-174. MR 1754775 (2001d:22008)

[21] G. A. Margulis and G. M. Tomanov, Invariant measures for actions of unipotent groups over local fields on homogeneous spaces, Invent. Math. 116 (1994), no. 1-3, 347-392, DOI 10.1007/BF01231565. MR1253197(95k:22013)

[22] S. P. Meyn and R. L. Tweedie, Markov chains and stochastic stability, Communications and Control Engineering Series, Springer-Verlag London Ltd., London, 1993. MR.1287609 (95j:60103)

[23] J.-F. Quint, Cônes limites des sous-groupes discrets des groupes réductifs sur un corps local, Transform. Groups 7 (2002), no. 3, 247-266, DOI 10.1007/s00031-002-0013-2 (French, with English summary). MR1923973 (2003h:20086)

[24] M. S. Raghunathan, Discrete subgroups of Lie groups, Springer-Verlag, New York, 1972. Ergebnisse der Mathematik und ihrer Grenzgebiete, Band 68. MR0507234 (58 \#22394a)

[25] Marina Ratner, On Raghunathan's measure conjecture, Ann. of Math. (2) 134 (1991), no. 3, 545-607, DOI 10.2307/2944357. MR1135878 (93a:22009)

[26] Marina Ratner, Raghunathan's conjectures for Cartesian products of real and p-adic Lie groups, Duke Math. J. 77 (1995), no. 2, 275-382, DOI 10.1215/S0012-7094-95-07710-2. MR1321062 (96d:22015)

[27] J. Tits, Reductive groups over local fields, Automorphic forms, representations and $L$ functions (Proc. Sympos. Pure Math., Oregon State Univ., Corvallis, Ore., 1977), Part 1, Proc. Sympos. Pure Math., XXXIII, Amer. Math. Soc., Providence, R.I., 1979, pp. 29-69. MR.546588 (80h:20064)

[28] Robert J. Zimmer, Ergodic theory and semisimple groups, Monographs in Mathematics, vol. 81, Birkhäuser Verlag, Basel, 1984. MR776417 (86j:22014) 
CNRS, Université Paris-Sud Bat.425, 91405 Orsay, France

E-mail address: yves.benoist@math.u-psud.fr

CNRS - Université Paris-Nord, LAGA, 93430 Villetaneuse, France

E-mail address: quint@math.univ-paris13.fr 